\title{
An Oligomer-Based Approach to Skeletal Diversity in Small-Molecule Synthesis
}

\section{Supporting Online Material}

David A. Spiegel, Frank C. Schroeder, ${ }^{a}$ Jeremy R. Duvall, and Stuart L. Schreiber

Howard Hughes Medical Institute, Department of Chemistry and Chemical Biology, Harvard University, Broad Institute of Harvard and MIT, Cambridge, Massachusetts, 02142

${ }^{a}$ Department of Biological Chemistry and Molecular Pharmacology, Harvard Medical School, Boston, Massachusetts 02115

\section{General Experimental Procedures.}

Unless stated otherwise, reactions were performed in flame dried glassware under a nitrogen or argon atmosphere. For all Mitsunobu reactions, alcohol and sulfonamide components were dried azeotropically with benzene before dissolution in THF. Solvents were passed through two activated alumina columns to remove impurities prior to use (as described in Organometallics, 1996, 15, 1518-1520). Amano Lipase PS from Pseudomonas cepacia was 
purchased from Sigma-Aldrich, St. Louis, MO. All other commercially obtained reagents were used as received.

Unless stated otherwise, all reactions were magnetically stirred and monitored by thin-layer chromatography (TLC) using E. Merck silica gel 60 F254 precoated plates $(0.25 \mathrm{~mm})$. Column or flash chromatography was performed with the indicated solvents using either silica gel (230-400 mesh) purchased from Bodman or pre-packed silica gel columns purchased from Biotage (Charlottesville, VA). Infrared spectra were recorded on a Nicolet Avatar 370 DTGS FTIR. ${ }^{1} \mathrm{H}$ and ${ }^{13} \mathrm{C}$ NMR spectra were recorded on Varian Mercury 400, Varian Mercury 500 and Varian Unity/Inova 600 spectrometers at $25^{\circ} \mathrm{C}$ using chloroform-d as solvent. In some cases, additional NMR spectra were acquired using acetone- $d_{6}$. Chemical shifts are reported relative to internal chloroform $\left({ }^{1} \mathrm{H}\right.$, $\delta 7.26$ ppm; ${ }^{13} \mathrm{C}, \delta 77.0$ ppm). Compounds A7, 15, epi-15, 18, 19-high, 19-low, 20, 21, and 22 were characterized in detail via phase-sensitive dqf-COSY, $\left({ }^{1} \mathrm{H},{ }^{13} \mathrm{C}\right)-\mathrm{HSQC}$, and $\left({ }^{1} \mathrm{H},{ }^{13} \mathrm{C}\right)-\mathrm{HMBC}$ spectra acquired employing standard pulse sequences. Relative configurations were assigned on the basis of nuclear Overhauser effects observed in phase-sensitive NOESY or ROESY spectra, in conjunction with coupling constants obtained from the dqf-COSY spectra.

Optical rotations were measured using a $3 \mathrm{~mL}$ cell with a $1 \mathrm{dm}$ path length on a JASCO DIP 370 polarimeter. High resolution mass spectra were obtained with JEOL AX-505 and JEOL SX-102 spectrometers. High performance liquid chromatography (HPLC) was performed on a Gilson 322 solvent delivery system using an Xterra Prep MS C18 OBD column. Chiral SFC chromatography was 
performed using a Chiralpak® $A D-H \quad S F C{ }^{T M}$ column purchased from Chiral Technologies, Inc.

\section{Experimental Section.}

Preparation of Alkyne 2. This material was synthesized following the route depicted in Scheme A1.

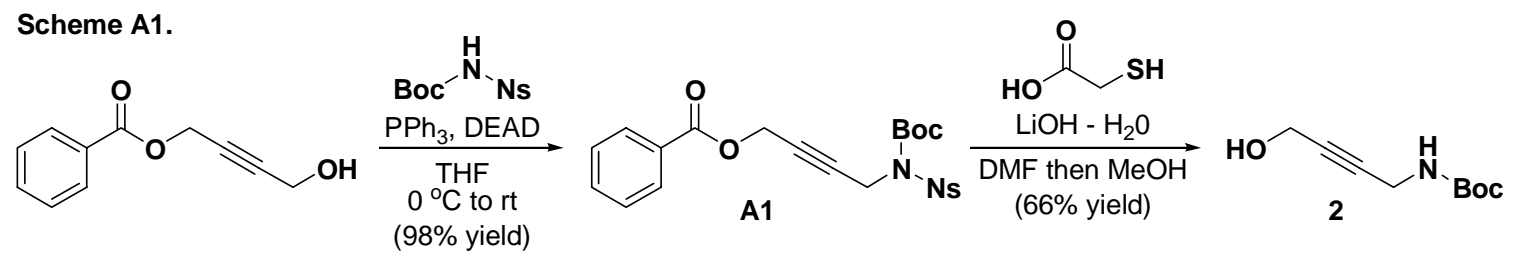

\section{4-(N-(tert-butoxycarbonyl)-2-nitrophenylsulfonamido)but-2-ynyl benzoate}

(A1). To a stirred solution of 4-benzoyloxy-but-2-yn-1-ol (3952 mg, $21 \mathrm{mmol}, 1.0$ equiv - prepared as described in Lloyd, R. C., et al.; Tetrahedron, 2004, 60, 717) in THF (105 mL) was added $\mathrm{PPh}_{3}(8279 \mathrm{mg}, 31.6 \mathrm{mmol}, 1.5$ equiv) and $\mathrm{N}$-tertbutoxycarbonyl-2-nitrobenzenesulfonamide $(6405 \mathrm{mg}, 21.2 \mathrm{mmol}, 1.01$ equiv prepared as described in Barret, S., et al.; Tetrahedron 2000, 56, 9633). The mixture was cooled to $0{ }^{\circ} \mathrm{C}$ and diethyl azodicarboxylate $(40 \%$ solution in toluene, $13.6 \mathrm{~mL}, 31.6 \mathrm{mmol}, 1.5$ equiv) was added dropwise over 3-5 minutes. The reaction was allowed to warm gradually to room temperature over approximately 48 hours and the solution was concentrated under reduced pressure and purified by flash chromatography (gradient elution, $0 \%$ to $25 \%$ 
EtOAc in hexanes) to yield intermediate $\mathbf{A} 1$ (9800 $\mathrm{mg}, 98 \%$ yield) as a white solid possessing the following spectral characteristics: IR (thin film/ $\mathrm{NaCl}$ ) 2981 (w), 1723 (s), 1541 (s), 1452 (w), 1364 (s), 1312 (m), 1264 (s), $1176(\mathrm{~m}), 1148$ (s), $1123(\mathrm{~m}), 1095(\mathrm{~m}), 1069(\mathrm{~m}), 955(\mathrm{w}), 913(\mathrm{w}), 851(\mathrm{w}), 780(\mathrm{w}), 712(\mathrm{~s}) \mathrm{cm}^{-1}$; 1H NMR (400 MHz, CDCl3) $\delta$ ppm 8.31 - $8.38(\mathrm{~m}, 1 \mathrm{H}), 8.03-8.08(\mathrm{~m}, 1 \mathrm{H}), 7.71$ - $7.80(\mathrm{~m}, 3 \mathrm{H}), 7.53-7.60(\mathrm{~m}, 1 \mathrm{H}), 7.39-7.47$ (m, $2 \mathrm{H}), 4.97$ (t, J=1.83 Hz, 2 H), 4.61 (t, J=1.83 Hz, $2 \mathrm{H}), 1.36(\mathrm{~s}, 9 \mathrm{H}) ;{ }^{13} \mathrm{C}$ NMR (100 MHz, CDCl3) $\delta$ 165.8, 149.6, 147.8, 134.3, 133.2, 133.2, 133, 132, 129.8, 129.5, 128.4, 124.5, 85.8, 81.8, 78.1, 52.7, 36.9, 27.8; HRMS (ES+) $\mathrm{m} / \mathrm{z} 492.1423$ [calc'd for $\mathrm{C}_{22} \mathrm{H}_{26} \mathrm{~N}_{3} \mathrm{O}_{8} \mathrm{~S}$ $(\mathrm{M}+\mathrm{NH} 4)$ 492.1441].

4-tert-Butoxycarbonylamino-2-butyn-1-ol (2). Benzoyl ester A1 (2912 mg, 6.1 mmol, 1.0 equiv), thioglycolic acid ( $0.85 \mathrm{~mL}, 12.3 \mathrm{mmol}, 2.0$ equiv), and $\mathrm{LiOH}$ (2060 mg, $49 \mathrm{mmol}, 8.0$ equiv) were dissolved in DMF (61 mL) and allowed to stir for 2 hours. At this point $\mathrm{MeOH}(50 \mathrm{~mL})$ was added and the mixture was allowed to stir for another 45 minutes. The reaction was quenched with a saturated aqueous solution of $\mathrm{NH}_{4} \mathrm{Cl}(10 \mathrm{~mL})$ and extracted with ethyl acetate (50 $\mathrm{mL} \times 3)$. The combined organic layers were washed with saturated $\mathrm{NaHCO}_{3}(25$ $\mathrm{mL} \times 2)$, water $(25 \mathrm{~mL} \times 1)$ and brine $(25 \mathrm{~mL} \times 1)$, dried over $\mathrm{Na}_{2} \mathrm{SO}_{4}$, filtered, and concentrated under reduced pressure. The residue was further purified by silica gel chromatography to provide 2 as a pale yellow oil $(754 \mathrm{mg}, 66 \%$ yield). IR (thin film/NaCl) 3337 (bs), 2978 (w), 2932 (w), 1687 (s), 1513 (m), 1453 (w), 1392 (w), 1366 (s), 1249 (s), 1157 (s), $1114(m), 1048(w), 1018(m), 931(w)$, 
827 (w), 779 (w) cm ${ }^{-1}$; $1 \mathrm{H}$ NMR (500 MHz, CDCl3) $\delta$ ppm 4.82 (br. s., 1 H) 4.25 (t, J=1.95 Hz, 2 H), 3.94 (br. s., 2 H), 2.37 (br. s., $1 \mathrm{H}$ ), 1.45 (s, $9 \mathrm{H}) ;{ }^{13} \mathrm{C} \mathrm{NMR}$ (125 MHz, CDCl3) $\delta 155.7,82.1,81.6,51.2,30.8,28.6$; HRMS (ES+) $\mathrm{m} / \mathrm{z}$ 186.1137 [calc'd for $\mathrm{C}_{9} \mathrm{H}_{16} \mathrm{NO}_{3}(\mathrm{M}+\mathrm{H})$ 186.1130].

Preparation of 4-(4-Bromophenylsulfonamido)but-2-ynyl benzoate (4). This material was synthesized following the route depicted in Scheme A2.

Scheme A2.

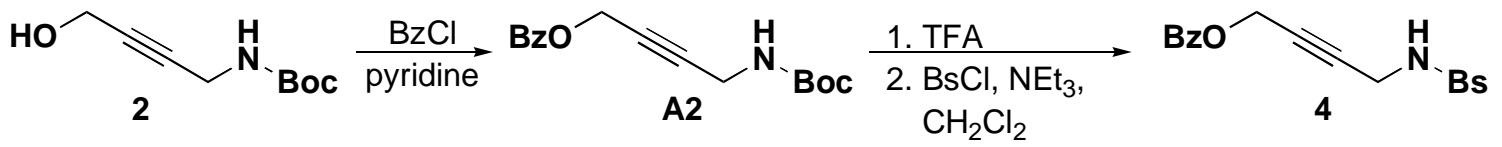

4-(tert-butoxycarbonylamino)but-2-ynyl benzoate (A2). To a stirred solution of 2 (56 mg, $0.3 \mathrm{mmol}, 1.0$ equiv) in pyridine $(0.6 \mathrm{~mL})$ was added benzoyl chloride (45 mg, $0.32 \mathrm{mmol}, 1.05$ equiv) by syringe. After 3 hours, the mixture was quenched with saturated aqueous $\mathrm{NaHCO}_{3}(3 \mathrm{~mL})$ and extracted with EtOAc (5 $\mathrm{mL} \times 4$ ). The organic layers were then combined, washed with aqueous $\mathrm{CuSO}_{4}$ (5 $\mathrm{mL} \times 2)$ then brine $(5 \mathrm{~mL} \times 1)$, dried over sodium sulfate, filtered, and evaporated under reduced pressure. The resulting residue was further purified by silica gel chromatography (gradient elution, 0 to $45 \%$ EtOAc in hexanes) to provide A2 (81 mg, 93\% yield) as a white solid; IR (thin film/ $\mathrm{NaCl}$ ) 3368 (bs), $2978(w), 1721(s), 1601(w), 1506(w), 1452(w), 1368(m), 1267(s), 1155(s)$, $1116(\mathrm{~m}), 1070(\mathrm{w}), 1026$ (w), $953(\mathrm{w}), 856(\mathrm{w}), 711(\mathrm{~s}) \mathrm{cm}^{-1}$; $1 \mathrm{H} \mathrm{NMR}(400 \mathrm{MHz}$, CDCl3) $\delta$ ppm $8.03-8.09(\mathrm{~m}, 2 \mathrm{H}), 7.58(\mathrm{t}, J=7.32 \mathrm{~Hz}, 1 \mathrm{H}), 7.45(\mathrm{t}, J=7.87 \mathrm{~Hz}, 2$ H), 4.92 (t, J=2.01 Hz, 2 H), 4.73 (br. s., 1 H), 3.99 (br. s., $2 \mathrm{H}), 1.44$ (s, $9 \mathrm{H}) ;{ }^{13} \mathrm{C}$ 
NMR $(100 \mathrm{MHz}, \mathrm{CDCl}) \delta$ $\delta 166.1,133.5,130,129.7,128.6,83.4,77.4,53,30.9$, 28.6; $\mathrm{HRMS}(\mathrm{ES}+) \mathrm{m} / \mathrm{z} 307.1661$ [calc'd for $\mathrm{C}_{16} \mathrm{H}_{23} \mathrm{~N}_{2} \mathrm{O}_{4}(\mathrm{M}+\mathrm{NH} 4)$ 307.1658].

\section{4-(4-Bromophenylsulfonamido)but-2-ynyl benzoate (4).}

To compound A2 (20 mg, $0.07 \mathrm{mmol}, 1.0$ equiv) was added trifluoroacetic acid $(0.7 \mathrm{~mL})$. Upon TFA addition, the solution began to bubble, which persisted for approximately 5 minutes. The reaction mixture was permitted to stir for an additional 15 minutes at which time the solvent was evaporated under reduced pressure and the residue was redissolved in $\mathrm{CH}_{2} \mathrm{Cl}_{2}(0.7 \mathrm{~mL})$. Brosyl chloride (27 mg, $0.1 \mathrm{mmol}, 1.5$ equiv) and triethylamine (21 mg, $0.21 \mathrm{mmol}, 3.0$ equiv) were then added, the mixture was stirred for 80 minutes, quenched with saturated aqueous $\mathrm{NH}_{4} \mathrm{Cl}(2 \mathrm{~mL})$ and brine $(2 \mathrm{~mL})$, and extracted with EtOAc (5 $m L \times 4$ ). Combined organic extracts were dried over sodium sulfate, filtered, concentrated under reduced pressure and purified chromatographically on silica gel (gradient elution, 0 to $85 \%$ EtOAc in hexanes) to yield 4-(4Bromophenylsulfonamido)but-2-ynyl benzoate $(4,20 \mathrm{mg}, 70 \%$ yield) as a white solid; IR (thin film/NaCl) 3275 (bs), 2925 (w), 2854 (s), 1772 (w), 1721 (m), 1575 (m), $1451(w), 1335(m), 1268(s), 1163(s), 1091(m), 1068(m), 1010(w), 820$ (w), $712(m), 610(w), 2981(w), 2360(w), 2341(m), 1727(s), 1542(s), 1366(s)$, $1312(\mathrm{w}), 1267$ (s), 1164 (s), $1123(\mathrm{~m}), 1069(\mathrm{~m}), 907$ (w), $760(\mathrm{~m}), 713(\mathrm{~m}) \mathrm{cm}^{-1}$; 1H NMR (500 MHz, CDCl3) $\delta$ ppm 8.03 (d, J=7.32 Hz, 2 H), 7.76 (d, J=8.79 Hz, 2 H), 7.64 (d, J=8.79 Hz, 2 H), 7.60 (t, J=7.32 Hz, 1 H), 7.47 (t, J=7.81 Hz, 2 H), 4.73 (t, J=5.86 Hz, $1 \mathrm{H}$ ), 4.70 (t, J=1.71 Hz, 2 H), 3.94 (ddd, J=5.98, 1.95, 1.83 
$\mathrm{Hz}, 2 \mathrm{H}) ;{ }^{13} \mathrm{C}$ NMR $(100 \mathrm{MHz}, \mathrm{CDCl} 3) \delta 166.6,138.8,133.9,132.6,130,129.2$, 128.8, 128.3, 81.1, 79.3, 52.8, 33.3; HRMS (ES+) $\mathrm{m} / \mathrm{z} 425.0165$ [calc'd for $\mathrm{C}_{17} \mathrm{H}_{18} \mathrm{BrN}_{2} \mathrm{O}_{4} \mathrm{~S}(\mathrm{M}+\mathrm{NH} 4)$ 425.0171].

\section{Preparation of $(R)-N$-Boc vinylglycinol $((R)-1)$ and (S)-N-Boc vinylglycinol}

$((S)-1)$. These compounds were synthesized through the route depicted in Scheme A3.
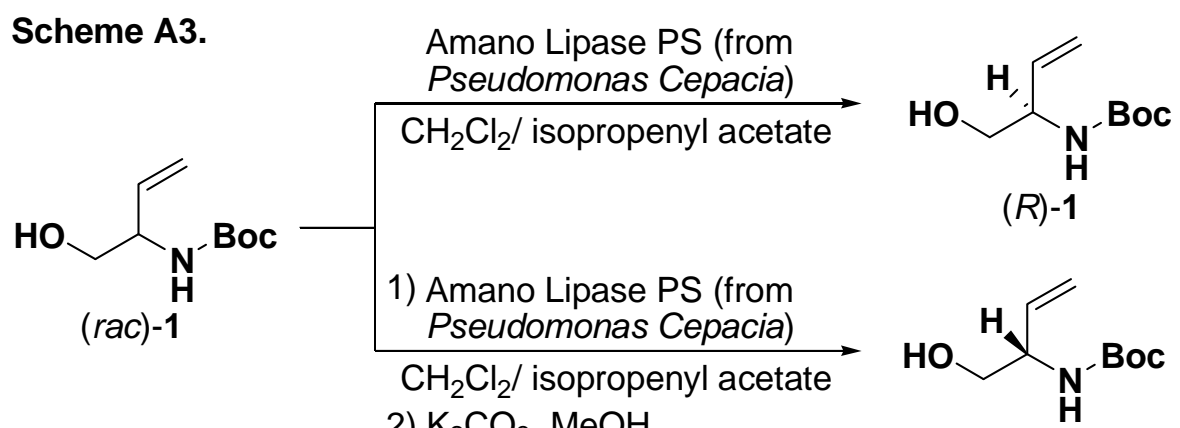

2) $\mathrm{K}_{2} \mathrm{CO}_{3}, \mathrm{MeOH}$

(S)-1

(rac)-, (R)-, and (S)-N-Boc vinylglycinol ((rac)-1, $(R)-1$, and (S)-1). Compounds (rac)-1 and (R)-1 were prepared according to the procedure in Sabat M.; Johnson C.J. Org. Lett. 2000, 2, 1089 (Ref. 17). Briefly, (rac)-1 was prepared in 4 steps and $37 \%$ overall yield starting from cis-butene-1,4-diol. This material (1671 mg, $8.9 \mathrm{mmol}, 1.0$ equiv) was then dissolved in a mixture of $\mathrm{CH}_{2} \mathrm{Cl}_{2}$ (13.7 $\mathrm{mL}$ ) and isopropenyl acetate $(13.7 \mathrm{~mL})$. To this solution was added Amano Lipase PS from Pseudomonas cepacia $(5.18 \mathrm{~g})$ and the slurry was stirred vigorously for 1 hour. At this time the reaction mixture was filtered through a plug of Celite $\AA^{\circledR}$ and the filtrate was concentrated under reduced pressure on a rotary evaporator. Chromatography on silica gel (gradient elution, 0 to $75 \%$ EtOAc in 
hexanes) provided $(R)-\mathbf{1}\left(753 \mathrm{mg}, 45 \%\right.$ yield) as a clear oil $[\alpha]_{D}{ }^{27}-30.2$ (c 0.6 , $\left.\mathrm{CHCl}_{3}\right)\left[\mathrm{Lit.}^{17}[\alpha]_{\mathrm{D}}{ }^{27}-28.5\left(\mathrm{c} 1.0 \mathrm{CHCl}_{3}\right)\right.$ and $(\mathrm{S})$-1-acetate $(984 \mathrm{mg}, 48 \%$ yield). The spectral properties of these materials were identical in all regards to those previously reported. ${ }^{14}$ The enantiomeric excess of $(R)-1$ was determined to be greater than $96 \%$ via conversion of this material to alcohol $\mathbf{A} 3$ (see procedure below) and analysis by chiral SFC chromatography. (S)-1 derived through this route was converted to (S)-A3 through an identical procedure, and the enantiomeric excess was determeined to be greater than $90 \%$.

The (S)-1-acetate (984 mg, $4.4 \mathrm{mmol}, 1.0$ equiv) was then dissolved in anhydrous $\mathrm{MeOH}$, and to this solution was added $\mathrm{K}_{2} \mathrm{CO}_{3}(2429 \mathrm{mg}, 17.6 \mathrm{mmol}$, 1.0 equiv) and the slurry was allowed to stir for $20 \mathrm{~min}$. At this time the reaction mixture was filtered over a pad of Celite $\AA$ and the solvent was removed under reduced pressure. The residue was then redissolved in EtOAc and washed with saturated aqueous ammonium chloride ( $x 1)$, and brine ( $x 1)$. Aqueous washings were extracted with EtOAc $(x 3)$, and the combined organic extracts were dried over sodium sulfate, filtered, and concentrated under reduced pressure to provide $(S)-1$ as a clear oil (666 mg, $81 \%$ yield) $[\alpha]_{D}^{27}+27.0$ (c 2.6, $\left.\mathrm{CHCl}_{3}\right)\left[\mathrm{Lit}^{14}\right.$ $[\alpha]_{D}^{27}+27.9\left(\mathrm{c} 1.0 \mathrm{CHCl}_{3}\right)$.

Preparation of (R)- and (S)- 4-Bromo-N-(1-hydroxybut-3-en-2yl)benzenesulfonamide (A3). 


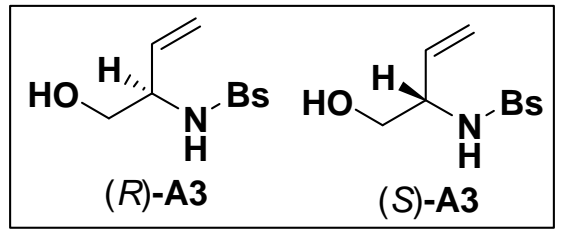

4-Bromo-N-(1-hydroxybut-3-en-2-yl)benzenesulfonamide ((R)-A3). To $(R)-N$-Boc vinylglycinol $((R)-1,27 \mathrm{mg}, 0.14 \mathrm{mmol}, 1.0$ equiv) was added trifluoroacetic acid $(0.2 \mathrm{~mL}, 2.9 \mathrm{mmol}, 20$ equiv). Upon TFA addition, the solution began to bubble, and after approximately 5 minutes, bubbling ceased. The reaction mixture was permitted to stir for an additional 20 minutes at which time the solvent was evaporated under reduced pressure and the residue was redissolved with a biphasic mixture of saturated aqueous $\mathrm{NaHCO}_{3}(2 \mathrm{~mL})$ and EtOAc ( $1 \mathrm{~mL}$ ). Brosyl chloride ( $55 \mathrm{mg}, 0.22 \mathrm{mmol}, 1.5$ equiv) was then added, the mixture was stirred vigorously for approximately 96 hours, and then quenched with saturated aqueous $\mathrm{NH}_{4} \mathrm{Cl}$. The organic phase was separated and the aqueous layer extracted with EtOAc $(5 \mathrm{~mL} \times 4)$. Combined organic extracts were dried over sodium sulfate, filtered, and concentrated under reduced pressure to provide a yellow oil which was further purified chromatographically on silica gel (gradient elution, 0 to $85 \%$ EtOAc in hexanes) to yield $(R)-N$-brosyl vinylglycinol ((R)-A3, $33.5 \mathrm{mg}, 76 \%$ yield) as a clear oil, $[\alpha]_{D}{ }^{24}-6.4\left(\mathrm{c} 1.5, \mathrm{CHCl}_{3}\right)$; mp 84-86 ${ }^{\circ} \mathrm{C}\left(\mathrm{CH}_{2} \mathrm{Cl}_{2}\right) ;$ IR (thin film/NaCl) 3497 (m), 3276 (m), 1575 (w), 1472 (w), 1434 (w), 1389 (w), 1329 (m), 1160 (s), 1091 (m), 1069 (w), 1010 (w) cm ; 1H NMR (500 MHz, CDCl3) $\delta$ ppm 7.74 (d, J=8.79 Hz, 2 H), 7.63 (d, J=9.00 Hz, 2 H), 5.60 (ddd, J=17.50, 10.00, $6.00 \mathrm{~Hz}, 1 \mathrm{H}$ ), 5.40 (d, J=7.81 Hz, $1 \mathrm{H}$ ), 5.11 $5.15(\mathrm{~m}, 1 \mathrm{H}), 5.10(\mathrm{~s}, 1 \mathrm{H}), 3.88-3.95(\mathrm{~m}, 1 \mathrm{H}), 3.65$ (dd, J=11.00, $4.00 \mathrm{~Hz}, 1$ 
H), 3.57 (dd, J=11.00, $6.00 \mathrm{~Hz}, 1 \mathrm{H}$ ), 2.22 (br. s., $1 \mathrm{H}$ ); ${ }^{13} \mathrm{C}$ NMR (125 MHz, $\mathrm{CDCl} 3) \delta 139.8,134.2,132.6,129,127.9,118.5,65,58 ; \mathrm{HRMS}(\mathrm{ES}+) \mathrm{m} / \mathrm{z}$ 307.9746 [calc'd for $\mathrm{C}_{23} \mathrm{H}_{19} \mathrm{BrO}_{4}(\mathrm{M}+\mathrm{H})$ 307.9740].

The enantiomeric excess of this material was determined to be $>96 \%$ by chiral SFC chromatography. A representative trace is shown below (racemic material gave 1:1 peak integrations, not shown):

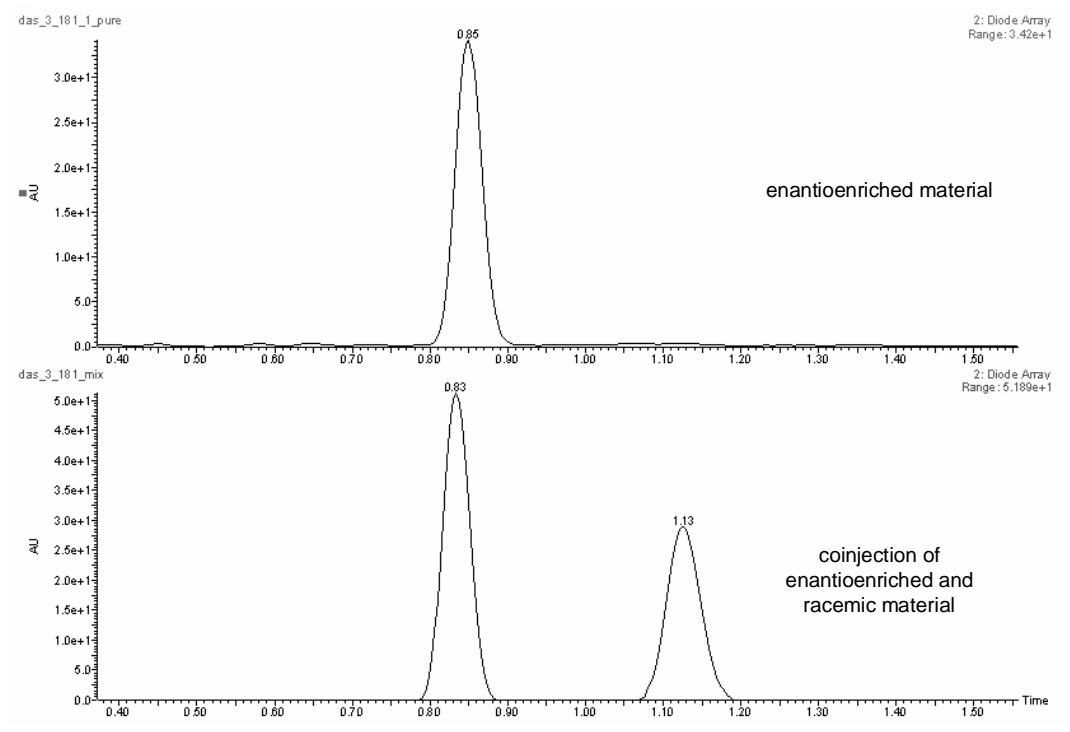

The identical procedure was followed starting from (S)-1 (27 mg, 0.14 mmol, 1.0 equiv) to provide (S)-A3 as a clear oil (30 mg, $68 \%$ yield).

\section{Alternative method for the preparation of enantioenriched alcohol (S)-A3.}

2-Trichloromethyl-4-vinyloxazoline (Ref. $17,38.0 \mathrm{~g}, 0.18 \mathrm{~mol}, 1.0$ equiv) was dissolved in $\mathrm{MeOH}(150 \mathrm{~mL})$. A $6 \mathrm{~N}$ aqueous solution of hydrochloric acid $(500 \mathrm{~mL})$ was then added and the solution was heated at $100{ }^{\circ} \mathrm{C}$ for $72 \mathrm{~h}$. Upon 
cooling to room temperature, an aliquot of this solution $(200 \mathrm{~mL}, 54 \mathrm{mmol}, 1.0$ equiv) was neutralized with solid $\mathrm{Na}_{2} \mathrm{CO}_{3}$. Additional $\mathrm{Na}_{2} \mathrm{CO}_{3}(5.7 \mathrm{~g}, 54 \mathrm{mmol}$, 1.0 equiv), 4-bromobenzenesulfonyl chloride (15.5 g, $60.5 \mathrm{mmol}, 1.12$ equiv), and EtOAc $(100 \mathrm{~mL})$ were then added and the biphasic mixture was allowed to stir vigorously for 48 hours. At this time, the aqueous phase was separated and extracted with EtOAc (100 mL x 1). Combined organic layers were dried over sodium sulfate, filtered, and evaporated under reduced pressure to provide a viscous brown oil. This material was further purified by chromatography on silica gel (gradient elution, 0 to $100 \%$ EtOAc in hexanes) to provide a white solid, which upon recrystallization from $\mathrm{CH}_{2} \mathrm{Cl}_{2}$, afforded (rac)-A3 $(8.2 \mathrm{~g}, 50 \%$ yield), whose spectral properties matched those reported above.

Racemic alcohol A3 (2841 mg, $9.3 \mathrm{mmol}, 1.0$ equiv) was then dissolved in $\mathrm{CH}_{2} \mathrm{Cl}_{2}(14 \mathrm{~mL})$ and isopropenyl acetate $(14 \mathrm{~mL})$. To this solution was added Amano Lipase PS from Pseudomonas cepacia (5.18 g) and the slurry was stirred vigorously for $45 \mathrm{~min}$. At this time the reaction mixture was filtered through a plug of Celite $\AA$ and the filtrate was concentrated under reduced pressure on a rotary evaporator. Chromatography on silica gel (gradient elution, 0 to $80 \%$ EtOAc in hexanes) provided the intermediate (S)-A3-acetate as a yellow oil (1450 mg, 45\% yield). This material (1250 mg, $3.6 \mathrm{mmol}, 1.0$ equiv) was then dissolved in anhydrous $\mathrm{MeOH}$, and to this solution was added $\mathrm{K}_{2} \mathrm{CO}_{3}(2477 \mathrm{mg}$, $18.0 \mathrm{mmol}, 1.0$ equiv) and the slurry was allowed to stir for $1 \mathrm{~h}$. At this time the reaction mixture was filtered over a pad of Celite $\AA$ and the solvent was removed under reduced pressure. The residue was then redissolved in EtOAc and 
washed with saturated aqueous ammonium chloride (x1), and brine (x1). Aqueous washings were extracted with EtOAc (x3), and the combined organic extracts were dried over sodium sulfate, filtered, and concentrated under reduced pressure to provide (S)-A3 as a white solid. The enantiomeric excess of this material was determined to be $>96 \%$ by chiral SFC chromatography. A representative trace is shown below (racemic material gave 1:1 peak integrations, not shown):

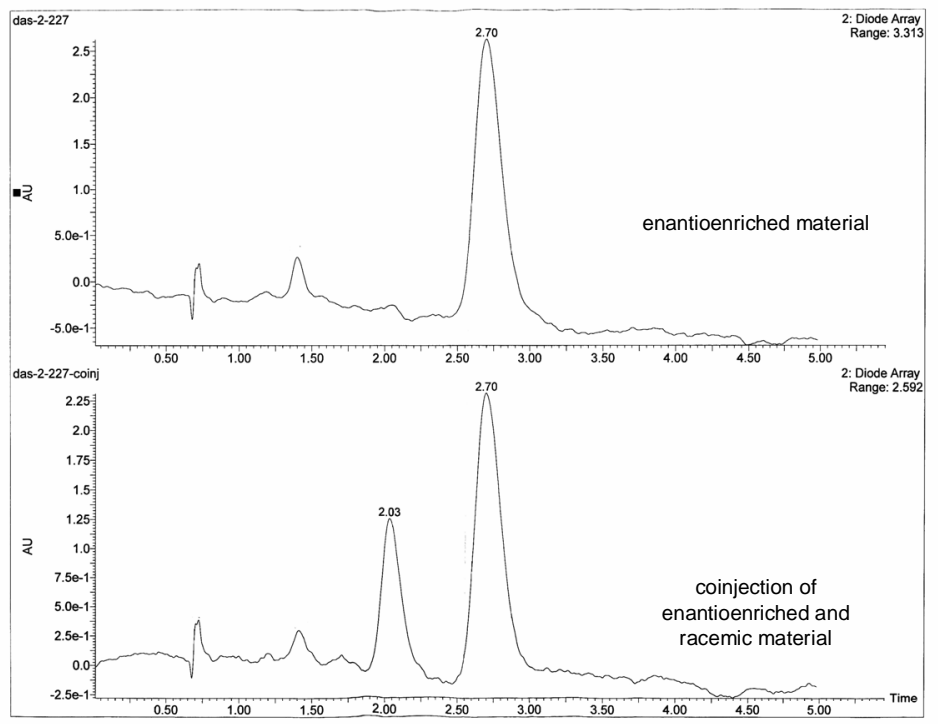

Preparation of $(R)$ - and (S)-2-(4-Bromophenylsulfonamido)but-3-enyl benzoate $((R)-3$ and $(S)-3)$.

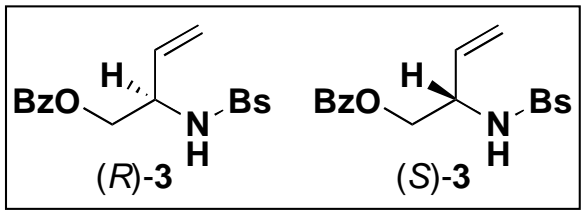

(S)-2-(4-Bromophenylsulfonamido)but-3-enyl benzoate ((S)-3). 
To a stirred solution of $(S)-N$-Brosyl vinylglycinol ((S)-A3, $177 \mathrm{mg}, 0.58 \mathrm{mmol}, 1.0$ equiv) in pyridine $(0.6 \mathrm{~mL})$ was added benzoyl chloride $(83 \mathrm{mg}, 0.59 \mathrm{mmol}, 1.02$ equiv) by syringe. After 3 hours, the mixture was quenched with saturated aqueous $\mathrm{NaHCO}_{3}(3 \mathrm{~mL})$ and extracted with EtOAc $(5 \mathrm{~mL} \times 4)$. The organic layers were then combined, washed with aqueous $\mathrm{CuSO}_{4}(5 \mathrm{~mL} \times 2)$ then brine (5 $\mathrm{mL} \times 1$ ), dried over sodium sulfate, filtered, and evaporated under reduced pressure. The resulting residue was further purified by silica gel chromatography (gradient elution, 0 to $60 \%$ EtOAc in hexanes) to provide $(S)-3(193 \mathrm{mg}, 81 \%$ yield) as a white crystalline solid, $[\alpha]_{\mathrm{D}}{ }^{27}+11.7\left(\mathrm{c} \quad 0.8, \mathrm{CH}_{2} \mathrm{Cl}_{2}\right) ; \mathrm{mp} 83-85{ }^{\circ} \mathrm{C}$ (hexanes/EtOAc); IR (powder) 3290 (m), 3068 (w), 2957 (w), 2360 (w), 1711 (s), $1600(w), 1573(m), 1432(m), 1336(m), 1273(s), 1164(m), 1119(m), 1089(s)$, $1025(\mathrm{~m}), 738(\mathrm{~m}), 711$ (s) $\mathrm{cm}^{-1} ; 1 \mathrm{H}$ NMR $(400 \mathrm{MHz}, \mathrm{CDCl} 3) \delta \mathrm{ppm} 7.85-7.92$ (m, $2 H), 7.64-7.71(m, 2 H), 7.56-7.63(m, 1 H), 7.41-7.50(m, 4 H), 5.74$ (ddd, J=17.02, 10.43, $5.49 \mathrm{~Hz}, 1 \mathrm{H}$ ), $5.20-5.36$ (m, $2 \mathrm{H}$ ), 5.11 (d, J=6.22 Hz, 1 $\mathrm{H}), 4.31-4.39(\mathrm{~m}, 1 \mathrm{H}), 4.22-4.30(\mathrm{~m}, 2 \mathrm{H}) ;{ }^{13} \mathrm{C} \mathrm{NMR}(100 \mathrm{MHz}, \mathrm{CDCl} 3) \delta$ 166.6, 139.9, 133.8, 132.5, 129.8, 129.4, 128.7, 128.7, 127.8, 118.8, 65.9, 55.8; HRMS (ES+) $m / z 427.0332$ [calc'd for $\mathrm{C}_{17} \mathrm{H}_{20} \mathrm{BrN}_{2} \mathrm{O}_{4} \mathrm{~S}(\mathrm{M}+\mathrm{NH} 4)$ 427.0327].

(R)-2-(4-Bromophenylsulfonamido)but-3-enyl benzoate $\quad((R)-3) . \quad$ This material was prepared through an identical procedure as its enantiomer (above) to provide a white crystalline solid, $[\alpha]_{D}^{27}-16.1(\mathrm{c} 1.0, \mathrm{MeOH})$.

Procedure for Mitsunobu Oligomerization Sequence (Scheme 2). To a stirred solution of sulfonamide (1.0 equiv), alcohol (2.5 equiv), and $\mathrm{PPh}_{3}$ (2.5 
equiv) in THF (0.2 $\mathrm{M}$ in sulfonamide) cooled to $0{ }^{\circ} \mathrm{C}$ in an ice water bath was added diethyl azodicarboxylate (40\% solution in toluene, 2.5 equiv) dropwise over 3-5 minutes. The reaction was allowed to warm gradually to room temperature over approximately 48 hours at which time, if thin-layer chromatography (TLC) analysis indicated completion, the solution was concentrated under reduced pressure and purified by flash chromatography (gradient elutions, $\mathrm{CH}_{3} \mathrm{CN}$ in $\mathrm{CH}_{2} \mathrm{Cl}_{2}$ ) to yield coupling products in the yields indicated in Schemes 1 and 3. For reactions that were not judged to be complete by TLC after 48 hours, the solution was re-cooled to $0{ }^{\circ} \mathrm{C}$, an additional 2.5 equivalents of alcohol, $\mathrm{PPh}_{3}$, and diethyl azodicarboxylate solution were added, and the reaction mixture was allowed to warm to room temperature over 48 hours. This procedure was repeated either until TLC indicated consumption of starting material or until a total of 10 equivalents of alcohol was added.

At this point, ene-ene combination $(S, R)-5$ was isolated after solvent evaporation and multiple rounds of flash chromatography along with recovered starting material (see below). For ene-ene combinations $(R, S)-,(R, R)^{-}$, and $(S, S)-5$, which were not judged to be complete by TLC at this point, reactions were re-cooled to $0{ }^{\circ} \mathrm{C}$ and 1,2,3-trimethoxybenzyl alcohol (2.5 equiv), $\mathrm{PPh}_{3}$ (2.5 equiv), and diethyl azodicarboxylate (40\% solution in toluene, 2.5 equiv) were added. After warming to room temperature over 24 hours, starting material had disappeared by TLC in all cases, solvent was evaporated, and the residue was purified via flash chromatography to yield the indicated coupled products (5) and trimethoxybenzylamine $\mathbf{A 4}$. 
Spectral data for $(S, R),(R, S),(R, R)$, and $(R, S)-\mathrm{N}-B o c-e n e-e n e((S, R)-,(R, S)-$, $(R, R)-$, and $(S, S)-5)$.

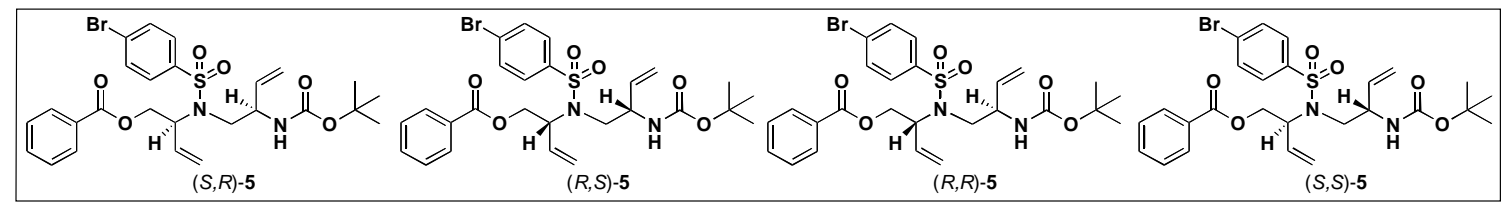

$(\boldsymbol{S}, \boldsymbol{R})$-N-Boc-ene-ene $((\boldsymbol{S}, \boldsymbol{R})-5)$. Isolated as a colorless oil $(36 \mathrm{mg}$ isolated product plus $53 \mathrm{mg}$ starting material recovered; $56 \%$ yield based on recovered starting material), $[\alpha]_{D}{ }^{30}-16.7$ (c 0.9, $\mathrm{CH}_{2} \mathrm{Cl}_{2}$ ); IR (thin film/ $\left.\mathrm{NaCl}\right) 3392$ (bs), 2977 (w), $2929(w), 1708(s), 1573(w), 1505(m), 1471(w), 1364(m), 1341(m), 1269$ (s), $1157(\mathrm{~s}), 1087(\mathrm{~m}), 1068(\mathrm{~m}), 1025(\mathrm{w}), 923(\mathrm{~m}), 822(\mathrm{~m}), 771(\mathrm{w}), 736(\mathrm{w})$, 711 (s) $\mathrm{cm}^{-1}$; $1 \mathrm{H}$ NMR $(400 \mathrm{MHz}, \mathrm{CDCl} 3) \delta$ ppm 7.91 (d, J=7.32 Hz, $\left.2 \mathrm{H}\right), 7.68$ (d, J=8.79 Hz, 2 H), 7.56 (t, J=7.51 Hz, 1 H), 7.48 (d, J=8.42 Hz, 2 H), 7.42 (t, $J=7.69 \mathrm{~Hz}, 2 \mathrm{H}), 5.67-5.81(\mathrm{~m}, \mathrm{~J}=17.30,14.01,5.49,5.31 \mathrm{~Hz}, 2 \mathrm{H}), 5.16-5.34$ (m, 5 H), $4.61-4.69$ (m, 2 H), $4.32-4.40$ (m, 2 H), 3.44 (dd, J=15.01, 9.89 Hz, 1 H), 3.17 (dd, J=15.01, $5.49 \mathrm{~Hz}, 1 \mathrm{H}), 1.41$ (s, $9 \mathrm{H}) ;{ }^{13} \mathrm{C} \mathrm{NMR} \mathrm{(100} \mathrm{MHz,} \mathrm{CDCl3)} \delta$ 166.2, 155.5, 139.2, 135.9, 133.4, 132.6, 132.3, 130, 129.7, 129.1, 128.7, 128, 120.9, 117.2, 79.9, 63.8, 58.8, 53.2, 48.2, 28.6; HRMS (ES+) $m / z 596.1412$ [calc'd for $\mathrm{C}_{26} \mathrm{H}_{35} \mathrm{BrN}_{3} \mathrm{O}_{6} \mathrm{~S}(\mathrm{M}+\mathrm{NH} 4)$ 596.1430].

$(\boldsymbol{R}, \boldsymbol{S})-\mathrm{N}$-Boc-ene-ene $((\boldsymbol{R}, \boldsymbol{S})-5)$. Isolated as a colorless oil $(10 \mathrm{mg}$ isolated product plus $50 \mathrm{mg}$ derivative $\mathbf{A 4} ; 68 \%$ yield based on recovered starting material), $[\alpha]_{D}^{30}+18.4\left(\right.$ c $\left.0.23, \mathrm{CH}_{2} \mathrm{Cl}_{2}\right)$

$(\boldsymbol{R}, \boldsymbol{R})$-N-Boc-ene-ene $((\boldsymbol{R}, \boldsymbol{R})-5)$. Isolated as a colorless oil $(21 \mathrm{mg}$ isolated product plus $47 \mathrm{mg}$ derivative $\mathbf{A 4} ; \mathbf{7 9} \%$ yield based on recovered starting 
material), $[\alpha]_{D}^{27}-1.3$ (c $0.60, \mathrm{CH}_{2} \mathrm{Cl}_{2}$ ); $1 \mathrm{H}$ NMR (500 MHz, CDCL3) $\delta$ ppm 7.78 (d, J=7.8 Hz, 2 H), 7.64 (d, J=8.3 Hz, 2 H), 7.57 (t, J=7.6 Hz, 1 H), 7.38 - 7.47 (m, $4 \mathrm{H}), 5.75$ - $5.88(\mathrm{~m}, 2 \mathrm{H}), 5.10$ - $5.36(\mathrm{~m}, 5 \mathrm{H}), 4.66$ - $4.75(\mathrm{~m}, 1 \mathrm{H}), 4.60$ (dd, J=12.0, $5.6 \mathrm{~Hz}, 1 \mathrm{H}), 4.30-4.42(\mathrm{~m}, 2 \mathrm{H}), 3.81-3.96$ (minor impurity, $0.8 \mathrm{H}$ ), $3.27-3.46(\mathrm{~m}, 2 \mathrm{H}), 1.43(\mathrm{~s}, 9 \mathrm{H})$; LRMS (ES+) $\mathrm{m} / \mathrm{z} 579.84$ [calc'd for $\mathrm{C}_{26} \mathrm{H}_{31} \mathrm{BrN}_{2} \mathrm{O}_{6} \mathrm{~S}(\mathrm{M}+\mathrm{H})$ 579.84].

$(\mathbf{S}, \mathbf{S})-\mathrm{N}$-Boc-ene-ene $((\boldsymbol{S}, \boldsymbol{S})-5)$. Isolated as a colorless oil $(25 \mathrm{mg}$ isolated product plus $97 \mathrm{mg}$ starting material recovered; $72 \%$ yield based on recovered derivative A4), $[\alpha]_{\mathrm{D}}^{27}+1.2\left(\mathrm{c} 0.40, \mathrm{CH}_{2} \mathrm{Cl}_{2}\right)$.

Spectral data for (R)- and (S)-2-(4-bromo-N-(3,4,5-trimethoxybenzyl)phenylsulfonamido)but-3-enyl benzoate ((R)- and (S)-A4).

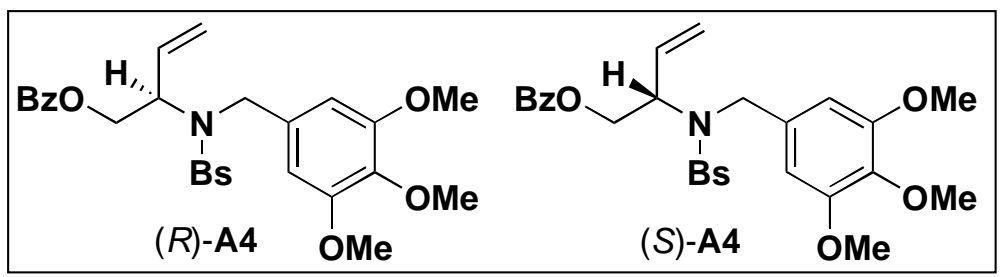

Spectral data for (R)-2-(4-bromo-N-(3,4,5-trimethoxybenzyl)phenylsulfonamido)but-3-enyl benzoate $((\boldsymbol{R})$ - A4). Isolated in the indicated yields as a colorless oil, $[\alpha]_{D}^{27}+36.6$ (c 0.5, MeOH); IR (thin film/NaCl) $3086(w), 3065$ (w), $2956(m), 2937(m), 2838(m), 1716(s), 1592(w), 1574(w), 1506(w), 1457(m)$, $1421(w), 1328(m), 1269(m), 1154(w), 1123(s), 1089(m), 1026(w), 1007(m)$, $922\left(\right.$ w) $\mathrm{cm}^{-1}$; $1 \mathrm{H}$ NMR (400 MHz, CDCL3) $\delta$ ppm $7.92(\mathrm{~d}, J=7.0 \mathrm{~Hz}, 2 \mathrm{H}), 7.62$ 7.70 (m, 2 H), 7.50 - $7.62(\mathrm{~m}, 3 \mathrm{H}), 7.44$ (t, J=7.7 Hz, 2 H), 6.48 (s, $2 \mathrm{H}), 5.63$ $5.74(\mathrm{~m}, 1 \mathrm{H}), 5.30(\mathrm{~d}, \mathrm{~J}=10.6 \mathrm{~Hz}, 1 \mathrm{H}), 5.20(\mathrm{~d}, \mathrm{~J}=17.2 \mathrm{~Hz}, 1 \mathrm{H}), 4.83-4.90(\mathrm{~m}$, 
$1 \mathrm{H}), 4.55(\mathrm{~d}, \mathrm{~J}=15.7 \mathrm{~Hz}, 1 \mathrm{H}), 4.16-4.39(\mathrm{~m}, 3 \mathrm{H}), 3.79(\mathrm{~s}, 3 \mathrm{H}), 3.68(\mathrm{~s}, 6 \mathrm{H})$;

${ }^{13} \mathrm{C}$ NMR (125 MHz, CDCl3). $\delta 166.1,153.5,140.3,133.5,132.5,132.5,132.4$, $129.9,129.7,128.9,128.7,127.7,120.7,105.4,63.9,61.1,59.3,56.2,49.3$; HRMS (ES+) m/z 607.1111 [calc'd for $\mathrm{C}_{27} \mathrm{H}_{28} \mathrm{BrNO}_{7} \mathrm{~S}(\mathrm{M}+\mathrm{NH} 4)$ 607.1114].

(S)-2-(4-bromo-N-(3,4,5-trimethoxybenzyl)phenylsul-fonamido)but-3-enyl benzoate ((S)- A4). Isolated in the indicated yields as a colorless oil, $[\alpha]_{D}-22.0$ (c $1.1, \mathrm{MeOH})$.

\section{Spectral data for $(R)$ - and (S)-N-Boc-ene-yne ((R)- and (S)-6).}

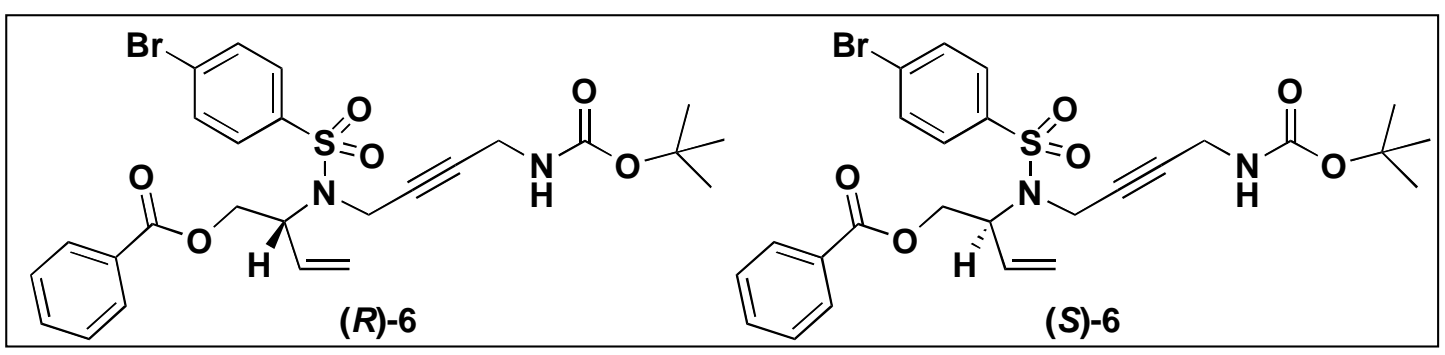

(S)-N-Boc-ene-yne 6. Isolated as a colorless oil (44.5 mg, $99 \%$ yield) $[\alpha]_{D}^{25}-1.1$

(c 1.0, MeOH); IR (thin film/NaCl) 3380 (bs), 2978 (w), 1715 (s), 1573 (w), 1504 (m), $1344(\mathrm{~m}), 1272(\mathrm{~s}), 1159(\mathrm{~s}), 1111(\mathrm{~m}), 1090(\mathrm{~m}), 1067(\mathrm{~m}), 1027(\mathrm{w}), 939$ (w), $881(w), 732(w), 713(\mathrm{~m}) \mathrm{cm}^{-1} ; 1 \mathrm{H}$ NMR $(500 \mathrm{MHz}, \mathrm{CDCl} 3) \delta \mathrm{ppm} 7.88(\mathrm{~d}$, J=7.32 Hz, $2 \mathrm{H}$ ), 7.76 (d, J=8.30 Hz, $2 \mathrm{H}), 7.58$ (t, J=7.57 Hz, $1 \mathrm{H}$ ), 7.54 (d, $J=8.30 \mathrm{~Hz}, 2 \mathrm{H}$ ), 7.44 (t, J=7.57 Hz, 2 H), 5.82 (ddd, J=17.09, 10.99, $5.62 \mathrm{~Hz}, 1$ H), 5.27 - $5.35(m, 2 H), 4.86-4.92(m, 1 H), 4.57$ (dd, J=11.80, 8.80 Hz, 2 H), 4.46 (dd, J=11.80, $5.30 \mathrm{~Hz}, 1 \mathrm{H}$ ), 4.28 (d, J=18.56 Hz, 1 H), 4.00 (d, J=18.56 Hz, $1 \mathrm{H}), 3.58$ - $3.73(\mathrm{~m}, 2 \mathrm{H}), 1.44(\mathrm{~s}, 9 \mathrm{H}) ;{ }^{13} \mathrm{C} \mathrm{NMR}(125 \mathrm{MHz}, \mathrm{CDCl} 3) \delta$ 166.4, 
$155.4,140,133.6,132.4,132,129.8,129.6,129.2,128.8,127.9,120.2,81.2$ 78.3, 63.5, 58.6, 33.9, 30.6, 28.6; HRMS (ES+) $\mathrm{m} / \mathrm{z} 599.0811$ [calc'd for $\mathrm{C}_{26} \mathrm{H}_{33} \mathrm{BrN}_{3} \mathrm{O}_{6} \mathrm{~S}(\mathrm{M}+\mathrm{NH} 4)$ 599.0827].

$(\boldsymbol{R})-\mathrm{N}-$ Boc-ene-yne $((\boldsymbol{R})-6)$. Isolated as a colorless oil, (126 mg, 98\% yield), $[\alpha]_{D}^{25}+1.4(\mathrm{c} 1.0, \mathrm{MeOH})$.

Spectral data for $(R)$ - and (S)-N-Boc-yne-ene ((R)- and (S)-7).

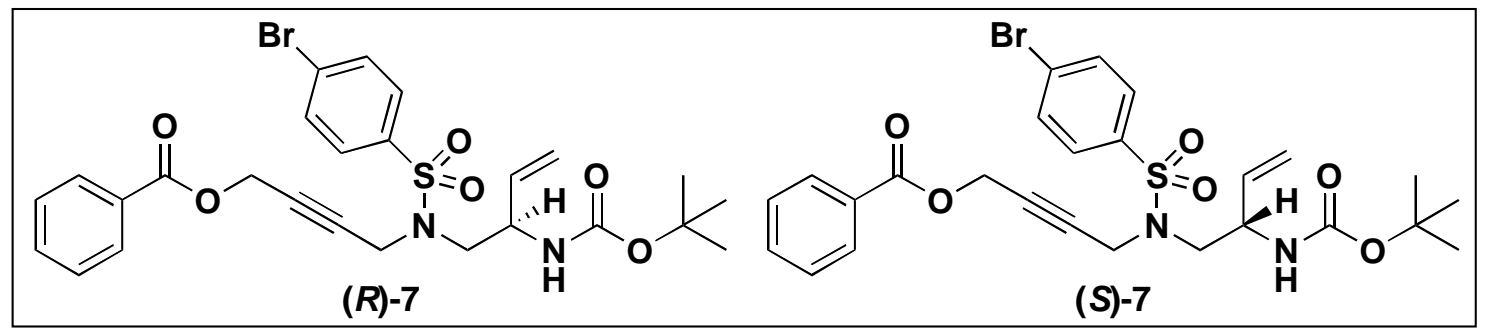

(rac)-N-Boc-yne-ene 7. Isolated as a white crystalline solid $(234 \mathrm{mg}, 85 \%$ yield); mp 106-108 ${ }^{\circ} \mathrm{C}$ (hexanes/EtOAc); IR (thin film/NaCl) 3389 (bs), 2978 (w), 2929 (w), 1720 (s), $1573(w), 1509(m), 1471(m), 1353(m), 1267(s), 1165(s)$, $1094(\mathrm{~m}), 1069(\mathrm{~m}), 1025(\mathrm{w}), 919(\mathrm{~m}), 823(\mathrm{w}), 765(\mathrm{~m}), 713(\mathrm{~m}) \mathrm{cm}^{-1} ; 1 \mathrm{H}$ NMR (600 MHz, CDCl3) $\delta$ ppm 8.02 (dd, J=8.3, $1.3 \mathrm{~Hz}, 2 \mathrm{H}$ ), 7.69 (d, J=8.5 Hz, $2 \mathrm{H}$ ), 7.59 - 7.62 (m, 3 H), 7.48 (t, J=7.9 Hz, 2 H), 5.76 (ddd, J=17.1, 10.5, 5.4 Hz, 1 H), 5.29 (d, J=17.0 Hz, 1 H), 5.20 (d, J=10.5 Hz, 1 H), 4.89 (br. s., 1 H), 4.68 (s, 2 H), $4.38(\mathrm{~d}, J=18.3 \mathrm{~Hz}, 2 \mathrm{H}), 4.20(\mathrm{~d}, J=18.7 \mathrm{~Hz}, 1 \mathrm{H}), 3.24$ - $3.33(\mathrm{~m}, 1 \mathrm{H}), 3.20$ (dd, J=14.0, $5.0 \mathrm{~Hz}, 1 \mathrm{H}), 1.45$ (s, $9 \mathrm{H}) ;{ }^{13} \mathrm{C} \mathrm{NMR}(100 \mathrm{MHz}, \mathrm{CDCl} 3) \delta$ 165.9, $155.8,138.1,135.6,133.7,132.5,130,129.5,129.4,128.8,128.2,117,80.9$, 79.3, 52.4, 49.9, 37.5, 28.6; HRMS (ES+) $m / z 577.0994$ [calc'd for $\mathrm{C}_{26} \mathrm{H}_{30} \mathrm{BrN}_{2} \mathrm{O}_{6}$ $(\mathrm{M}+\mathrm{H})$ 577.1008]. 
$(\boldsymbol{R})-\mathrm{N}-$ Boc-yne-ene $((\boldsymbol{R})-7)$. Isolated as a white solid, (118 $\mathrm{mg}, 82 \%$ yield), $[\alpha]_{\mathrm{D}}{ }^{25}$ -34.8 (c 1.0, MeOH).

(S)-N-Boc-yne-ene ((S)-7). Isolated as a white solid, (119 mg, 85\% yield), $[\alpha]_{D}{ }^{25}$ +32.4 (c 1.0, MeOH).

\section{Spectral data for N-Boc-yne-yne 8.}

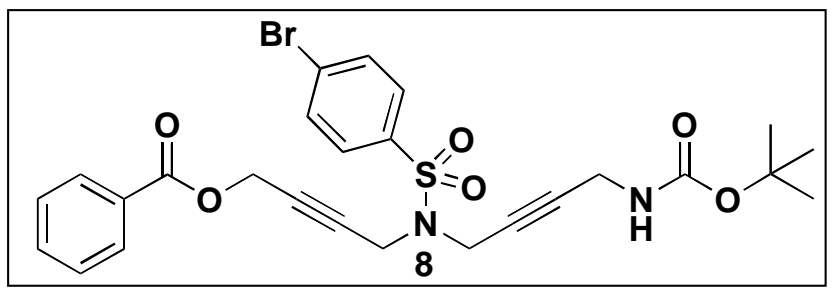

N-Boc-yne-yne 8. Isolated as a colorless oil (323 mg, 92\% yield); IR (thin film/NaCl) 3392 (bs), 2977 (w), 2929 (w), 1721 (s), 1573 (w), 1505 (w), 1452 (w), $1366(m), 1268(s), 1164(s), 1092(m), 1068(m), 1009(w), 906(w), 760(w)$, $711(\mathrm{~m}) \mathrm{cm}^{-1} ; 1 \mathrm{H} \mathrm{NMR}(400 \mathrm{MHz}, \mathrm{CDCl} 3) \delta \mathrm{ppm} 8.02(\mathrm{~d}, J=7.7 \mathrm{~Hz}, 2 \mathrm{H}), 7.67$ 7.70 (m, 2 H), 7.53 - 7.64 (m, 3 H), 7.45 (t, J=7.7 Hz, 2 H), 4.75 (s, 2 H), 4.67 (br. s., $1 \mathrm{H}$ ), 4.19 (s, $2 \mathrm{H}$ ), 4.12 (s, $2 \mathrm{H}$ ), 3.75 (br. s., $2 \mathrm{H}$ ), 1.42 (s, $9 \mathrm{H}) ;{ }^{13} \mathrm{C}$ NMR $(100 \mathrm{MHz}, \mathrm{CDCl} 3) \delta 165.9,155.4,137.6,133.7,132.4,130,129.6,129.5,128.8$ 128.3, 82.8, 80.5, 80.3, 79.4, 75.4, 64.5, 52.5, 37.1, 37, 28.6; HRMS (ES+) $m / z$ 592.1105 [calc'd for $\mathrm{C}_{26} \mathrm{H}_{31} \mathrm{BrN}_{3} \mathrm{O}_{6} \mathrm{~S}(\mathrm{M}+\mathrm{NH} 4)$ 592.1117].

General procedure for Ru-catalyzed metathesis reactions (Schemes 3 and

4). A solution of metathesis substrate (1.0 equiv) and $\left[\mathrm{RuCl}_{2}(=\mathrm{CHPh})(\mathrm{PCy})_{2}\right]$ (0.05 equiv) in benzene $(0.03 \mathrm{M})$ was placed under ethylene atmosphere by two freeze-pump-thaw cycles. This mixture was heated under reflux for 12-24 hours, 
cooled to room temperature, filtered over a plug of neutral alumina rinsing with $\mathrm{CH}_{2} \mathrm{Cl}_{2}$ and EtOAc, and solvent was then evaporated under reduced pressure. The resulting residue was purified by chromatography on silica gel (gradient elution, $\mathrm{CH}_{3} \mathrm{CN}$ in $\mathrm{CH}_{2} \mathrm{Cl}_{2}$ ) to provide products in the indicated yields.

Spectral data for tetrahydropyridines $(S, R)-,(R, S),(R, R)-$, and $(S, S)-9$.

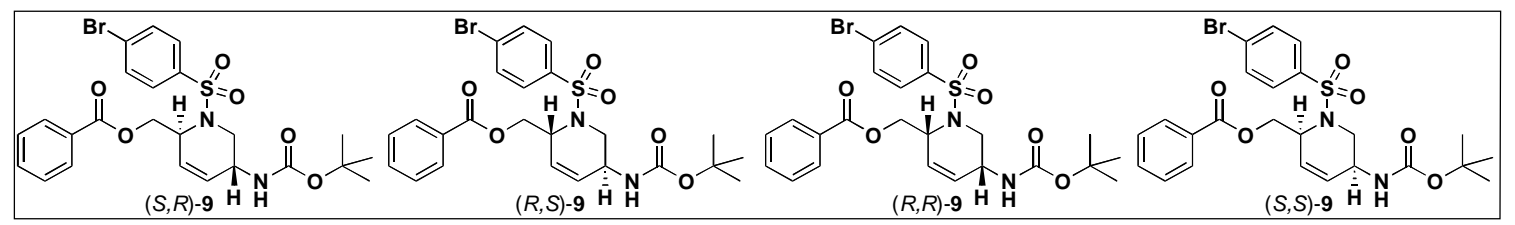

Tetrahydropyridine $(S, R)-9$. Isolated after $24 \mathrm{~h}$ reaction time as a colorless oil (11 mg, 79\% yield), $[\alpha]_{\mathrm{D}}{ }^{30}+67.0\left(\mathrm{c} 0.60, \mathrm{CH}_{2} \mathrm{Cl}_{2}\right)$; IR (thin film/NaCl) $3384(\mathrm{~m})$, 2975 (w), 2927 (w), 1701 (s), 1575 (w), 1500 (s), 1452 (w), 1330 (s), 1272 (s), 1157 (s), 1117 (s), 1068 (s), 1026 (w), 1009 (w), 970 (s), 918 (w), 820 (w), 746 (m), 709 (s) $\mathrm{cm}^{-1} ; 1 \mathrm{H} \mathrm{NMR} \mathrm{(400} \mathrm{MHz,} \mathrm{CDCl3)} \delta$ ppm 7.90 (d, J=7.7 Hz, $2 \mathrm{H}$ ), $7.65(\mathrm{~d}, J=8.8 \mathrm{~Hz}, 2 \mathrm{H}), 7.58(\mathrm{t}, \mathrm{J}=7.5 \mathrm{~Hz}, 1 \mathrm{H}), 7.41-7.51$ (m, $4 \mathrm{H}), 5.93-6.01$ (m, 2 H), 4.80 (br. s., 1 H), 4.51 (d, J=8.4 Hz, 1 H), 4.43 (dd, J=11.7, 6.6 Hz, 1 H), 4.29 (dd, J=11.8, $4.4 \mathrm{~Hz}, 1 \mathrm{H}), 4.05-4.15(\mathrm{~m}, 1 \mathrm{H}), 3.97$ (d, J=14.6 Hz, $1 \mathrm{H})$, 3.48 (dd, J=14.6, $3.3 \mathrm{~Hz}, 1 \mathrm{H}), 1.44(\mathrm{~s}, 9 \mathrm{H}) ;{ }^{13} \mathrm{C} \mathrm{NMR} \mathrm{(100} \mathrm{MHz,} \mathrm{CDCl3)} \delta$ 166.3, 155.2, 140, 133.6, 132.6, 129.8, 129.6, 128.7, 128.7, 127.9, 127.8, 80.2, 64, 52.4, 45.4, 43.6, 28.6; HRMS (ES+) $\mathrm{m} / 2568.1113$ [calc'd for $\mathrm{C}_{24} \mathrm{H}_{31} \mathrm{BrN}_{3} \mathrm{O}_{6} \mathrm{~S}$ $(\mathrm{M}+\mathrm{NH} 4)$ 568.1117].

Tetrahydropyridine $(\boldsymbol{R}, \boldsymbol{S})-9$. Isolated after $24 \mathrm{~h}$ reaction time as a colorless oil (4.1 mg, 85\% yield), $[\alpha]_{D}^{30}-65.6\left(\mathrm{c} 0.16, \mathrm{CH}_{2} \mathrm{Cl}_{2}\right.$ ). 
Tetrahydropyridine $(\boldsymbol{R}, \boldsymbol{R})-9$. Isolated after $24 \mathrm{~h}$ reaction time as a colorless oil (14 mg, 77\% yield), $[\alpha]_{D}{ }^{30}+7.3\left(\mathrm{c} 0.59, \mathrm{CH}_{2} \mathrm{Cl}_{2}\right.$ ). IR (thin film/NaCl) $3366(\mathrm{w})$, $2975(w), 2929(w), 1705(s), 1507(m), 1346(w), 1269(s), 1157(s), 1095(m)$, 1068 (m), 1047 (w), 1025 (m), 1009 (m), 982 (w) cm ${ }^{-1}$; 1H NMR (500 MHz, CDCL3) $\delta$ ppm 8.02 (d, J=7.3 Hz, 2 H), 7.71 (d, J=7.8 Hz, 2 H), 7.51 - 7.62 (m, 3 H), $7.46(t, J=7.8 \mathrm{~Hz}, 2 \mathrm{H}), 5.79(\mathrm{~d}, J=9.8 \mathrm{~Hz}, 1 \mathrm{H}), 5.70(\mathrm{~d}, J=10.1 \mathrm{~Hz}, 1 \mathrm{H}), 4.68$ (br. s., $1 \mathrm{H}), 4.44-4.53(\mathrm{~m}, 1 \mathrm{H}), 4.31-4.41(\mathrm{~m}, 2 \mathrm{H}), 4.17(\mathrm{~d}, \mathrm{~J}=13.2 \mathrm{~Hz}, 1 \mathrm{H})$, 3.92 (br. s., $1 \mathrm{H}), 2.87$ - 3.00 (m, $1 \mathrm{H}), 1.43$ (s, $9 \mathrm{H}) ;{ }^{13} \mathrm{C}$ NMR (125 MHz, CDCl3̧) $\delta 166.5,155,133.5,132.8,131,130,129.8,128.7,128.6,128,126,64.6,52.4$ 44.2, 43.5, 28.6; HRMS (ES+) $m / z 568.1118$ [calc'd for $\mathrm{C}_{24} \mathrm{H}_{31} \mathrm{BrN}_{3} \mathrm{O}_{6} \mathrm{~S}(\mathrm{M}+\mathrm{NH} 4)$ 568.1117].

Tetrahydropyridine $(\mathbf{S}, \mathbf{S})-\mathbf{9}$. Isolated after $24 \mathrm{~h}$ reaction time as a colorless oil (7 mg, $75 \%$ yield), $[\alpha]_{D}{ }^{30}-6.7$ (c $0.30, \mathrm{CH}_{2} \mathrm{Cl}_{2}$ ).

\section{Spectral data for dihydropyrroles $(R)$ - and $(S)-10$.}

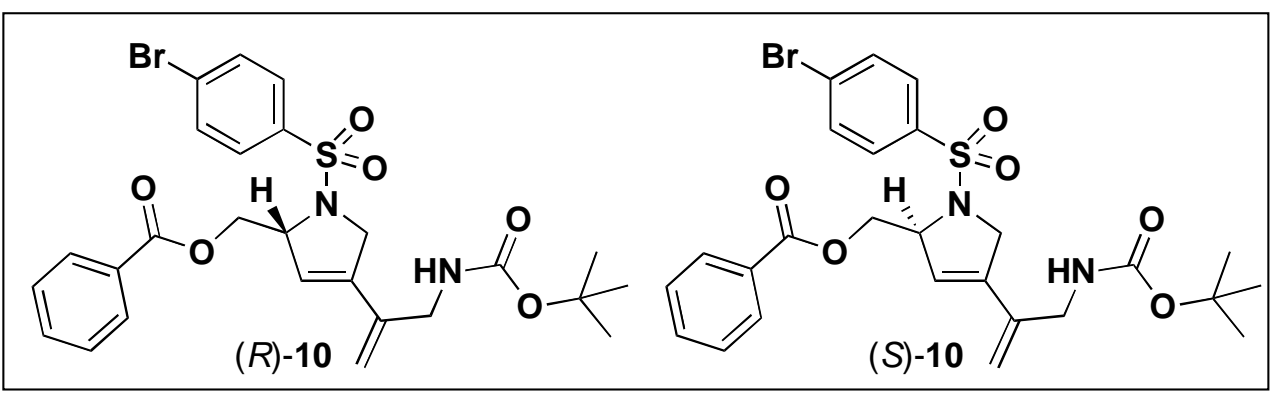

(rac)-Dihydropyrrole 10. Isolated after $16 \mathrm{~h}$ reaction time as a colorless oil (19 mg, 73\% yield); IR (thin film/NaCl) 3367 (bs), 2976 (w), 1707 (s), 1573 (m), 1513 (m), 1452 (w), 1364 (s), 1268 (s), 1159 (s), 1092 (s), 1068 (s), 1026 (w), 1009 
(w), $824(\mathrm{~m}), 740$ (s), $712(\mathrm{~s}) \mathrm{cm}^{-1}$; 1H NMR (400 MHz, CDCl3) $\delta$ ppm 7.87 - 8.05 (m, 2 H), 7.72 (d, J=8.3 Hz, 2 H), 7.52 - 7.66 (m, 3 H), 7.42 (t, J=7.8 Hz, 2 H), 5.77 (br. s., 1 H), 5.19 (s, 1 H), 4.94 (s, 1 H), 4.86 (br. s., 1 H), 4.66 (dd, J=11.1, $3.5 \mathrm{~Hz}, 1 \mathrm{H}$ ), 4.60 (br. s., $1 \mathrm{H}$ ), 4.42 (dd, J=11.1, $5.5 \mathrm{~Hz}, 1 \mathrm{H}), 4.20$ - 4.36 (m, 2 H), 3.87 (d, J=5.9 Hz, $2 \mathrm{H}$ ), 1.39 (s, $9 \mathrm{H}) ;{ }^{13} \mathrm{C}$ NMR (100 MHz, CDCl3) $\delta$ 166.3, $155.8,137.9,137.5,137,136.8,133.4,132.8,129.8,129,128.6,128.3,122.4$, 115.5, 80, 66.8, 66.5, 55.7, 43, 28.5; HRMS (ES+) $\mathrm{m} / \mathrm{z} 577.1000$ [calc'd for $\mathrm{C}_{26} \mathrm{H}_{30} \mathrm{BrN}_{2} \mathrm{O}_{6} \mathrm{~S}(\mathrm{M}+\mathrm{H})$ 577.1008].

(R)-Dihydropyrrole 10. Isolated as a colorless oil, (15 mg, 56\% yield), $[\alpha]_{D}^{25}$ -

11.5 (c $\left.0.6, \mathrm{CH}_{2} \mathrm{Cl}_{2}\right)$.

(S)-Dihydropyrrole 10. Isolated as a colorless oil, (18 mg, $58 \%$ yield), $[\alpha]_{D}{ }^{25}$ $+10.7\left(\mathrm{c} 0.4, \mathrm{CH}_{2} \mathrm{Cl}_{2}\right)$.

\section{Spectral data for Tetrahydropyridines (R)- and (S)-11.}

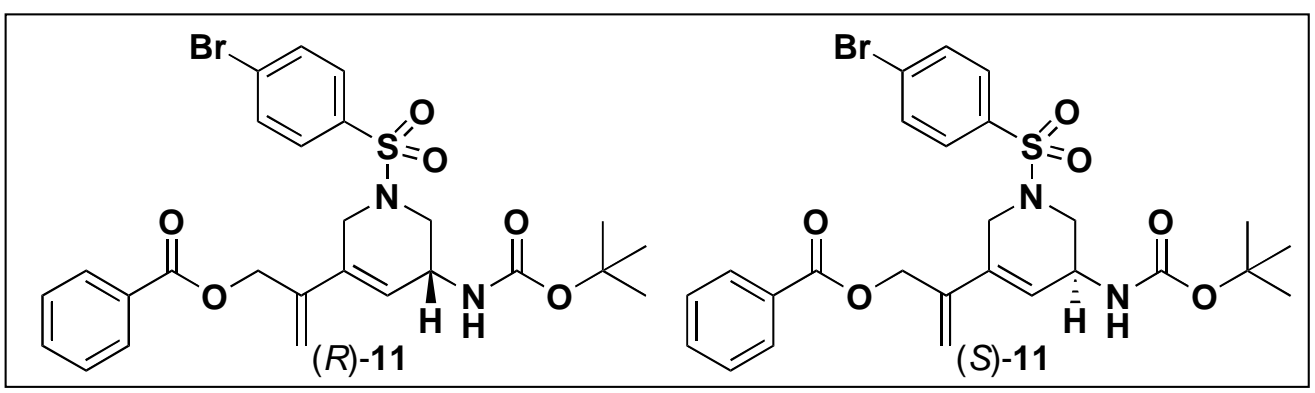

(rac)-Tetrahydropyridine 11 . Isolated after $24 \mathrm{~h}$ reaction time as a white solid (20 mg, 83\% yield); IR (thin film/NaCl) 3346 (m), 2925 (w), 1713 (s), 1669 (s), 1514 (s), 1467 (w), 1366 (s), 1280 (s), 1267 (s), 1158 (s), 1067 (s), 1028 (w), 824 (m), 751 (s), $710(\mathrm{~s}) \mathrm{cm}^{-1} ; 1 \mathrm{H}$ NMR $(400 \mathrm{MHz}, \mathrm{CDCl}) \delta$ ppm $7.88-8.08(\mathrm{~m}, 2$ H), 7.63 - $7.77(\mathrm{~m}, 4 \mathrm{H}), 7.58(\mathrm{t}, J=7.4 \mathrm{~Hz}, 1 \mathrm{H}), 7.45(\mathrm{t}, J=7.7 \mathrm{~Hz}, 2 \mathrm{H}), 5.97$ (d, 
J=3.9 Hz, $1 \mathrm{H}), 5.40(\mathrm{~s}, 1 \mathrm{H}), 5.25(\mathrm{~s}, 1 \mathrm{H}), 4.96(\mathrm{~s}, 2 \mathrm{H}), 4.81(\mathrm{~d}, J=8.8 \mathrm{~Hz}, 1 \mathrm{H})$, 4.37 (br. s., 1 H), 4.15 (d, J=15.9 Hz, 1 H), 3.57 (d, J=11.5 Hz, 1 H), 3.37 (d, $J=15.4 \mathrm{~Hz}, 1 \mathrm{H}), 2.85$ (dd, J=11.5, $2.7 \mathrm{~Hz}, 1 \mathrm{H}), 1.44(\mathrm{~s}, 9 \mathrm{H}) ;{ }^{13} \mathrm{C}$ NMR $(125$ $\mathrm{MHz}, \mathrm{CDCl} 3) \delta 166.2,155.2,139.1,133.7,133.5,132.8,130,129.9,129.3,129$, 128.7, 128.5, 123.3, 115.8, 80.3, 65.1, 48.5, 45.3, 45, 28.6; HRMS (ES+) $\mathrm{m} / \mathrm{z}$ 594.1257 [calc'd for $\mathrm{C}_{26} \mathrm{H}_{33} \mathrm{BrN}_{3} \mathrm{O}_{6} \mathrm{~S}(\mathrm{M}+\mathrm{NH} 4)$ 594.1273].

$(R)$ - Tetrahydropyridine 11 . Isolated as a white solid, (26 $\mathrm{mg}, 87 \%$ yield), $[\alpha]_{D}{ }^{25}$ $+22.2\left(\mathrm{c} 1.4, \mathrm{CH}_{2} \mathrm{Cl}_{2}\right)$.

(S)- Tetrahydropyridine 11 . Isolated as a white solid, (28 $\mathrm{mg}, 82 \%$ yield), $[\alpha]_{D}{ }^{25}$ -27.3 (c 1.0, $\left.\mathrm{CH}_{2} \mathrm{Cl}_{2}\right)$.

For chain-extension of 5-8, starting Boc amides were treated with trifluoroacetic acid (20 equiv). Upon TFA addition, the solution began to bubble, and after approximately 5 minutes, bubbling ceased. The reaction mixture was permitted to stir for an additional 60 minutes at which time the solvent was evaporated under reduced pressure and the residue was redissolved with a biphasic mixture of saturated aqueous $\mathrm{NaHCO}_{3}$ and EtOAc $(2: 1 \mathrm{v} / \mathrm{v})$. oNitrobenzenesulfonyl chloride (2.0 equiv) was then added, the mixture was stirred vigorously for approximately 96 hours, and then quenched with saturated aqueous $\mathrm{NH}_{4} \mathrm{Cl}$. The organic phase was separated and the aqueous layer extracted with EtOAc. Combined organic extracts were dried over sodium sulfate, filtered, concentrated under reduced pressure, and purified 
chromatographically on silica gel (gradient elution, $\mathrm{CH}_{3} \mathrm{CN}$ in $\mathrm{CH}_{2} \mathrm{Cl}_{2}$ ) to yield the following intermediate nosylates:

Spectral data for N-Ns-yne-yne A5.

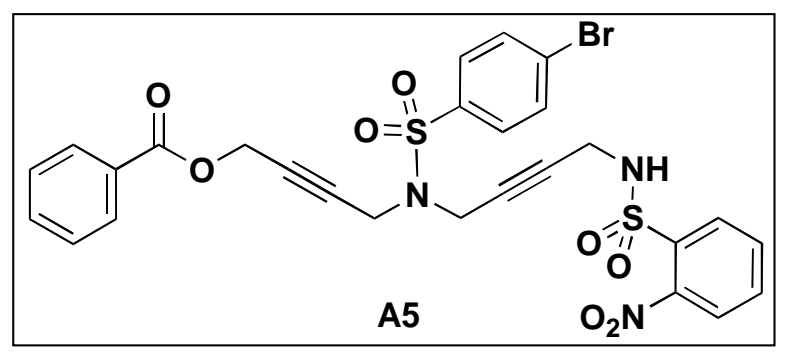

N-Ns-yne-yne A5. Isolated as a white solid; IR (thin film/NaCl) 3333 (bs), 1719 (m), 1573 (w), 1415 (w), 1348 (s), 1264 (s), 1161 (s), 1090 (s), 1067 (s), 1026 (w), $954(w), 904(m), 853(w), 728(m), 712(m) c^{-1}$; 1H NMR (400 MHz, CDCl3) $\delta$ ppm 8.12 - $8.18(\mathrm{~m}, 1 \mathrm{H}), 8.03(\mathrm{~d}, \mathrm{~J}=7.0 \mathrm{~Hz}, 2 \mathrm{H}), 7.89-7.95(\mathrm{~m}, 1 \mathrm{H})$, $7.76-7.83(\mathrm{~m}, 2 \mathrm{H}), 7.59-7.69(\mathrm{~m}, 5 \mathrm{H}), 7.48(\mathrm{t}, \mathrm{J}=7.7 \mathrm{~Hz}, 2 \mathrm{H}), 5.61$ (t, J=6.2 Hz, 1 H), 4.70 (t, J=2.0 Hz, 2 H), 3.98 (t, J=1.6 Hz, 2 H), 3.90 (dt, J=6.3, 2.0 Hz, 2 H), $3.80(t, J=1.8 \mathrm{~Hz}, 2 \mathrm{H}) ;{ }^{13} \mathrm{C}$ NMR $(100 \mathrm{MHz}, \mathrm{CDCl} 3) \delta 165.9,137.4,134.4$, 134, 133.8, 133.2, 132.5, 131.5, 130, 129.4, 128.8, 128.5, 125.9, 80.8, 80, 78.8, $77.8,52.4,36.8,36.6,33.6,166.4,139.7,134.1,134.1,133.8,133.2,132.5$, $131.8,131.5,129.8,129.5,129,128.8,128.6,128,125.7,120.4,80.6,78.7$, 63.3, 58.4, 33.6, 33.5; HRMS (ES+) $\mathrm{m} / 2677.0349$ [calc'd for $\mathrm{C}_{27} \mathrm{H}_{26} \mathrm{BrN}_{4} \mathrm{O}_{8} \mathrm{~S}_{2}$ $(\mathrm{M}+\mathrm{NH} 4)$ 677.0375].

\section{Spectral data for N-Ns-ene-yne A6.}




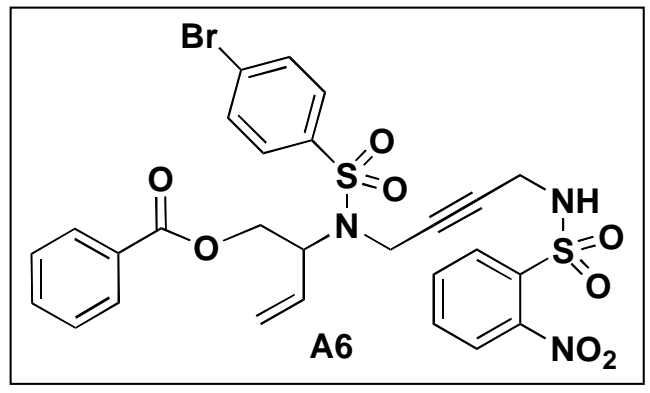

N-Ns-ene-yne A6. Non-racemic material was carried through this sequence but the optical rotation was not obtained. For more complete characterization the racemic material was synthesized whose characterization data is shown here. Isolated as a white solid; IR (thin film/NaCl) 3332 (bs), 3091 (w), 2924 (w), 1710 (m), 1573 (s), 1414 (w), 1341 (s), 1270 (s), 1223 (s), 1160 (s), 1111 (m), 1090 (s), $1067(\mathrm{~m}), 1027(\mathrm{w}), 939(\mathrm{w}), 881(\mathrm{~m}), 853(\mathrm{w}), 732(\mathrm{~m}), 712(\mathrm{~m}) \mathrm{cm}^{-1} ; 1 \mathrm{H}$ NMR $(500 \mathrm{MHz}, \mathrm{CDCl} 3) \delta \mathrm{ppm} 8.10-8.16(\mathrm{~m}, 1 \mathrm{H}), 7.87$ - $7.93(\mathrm{~m}, 1 \mathrm{H}), 7.84$ 7.87 (m, 2 H), 7.73 - 7.78 (m, 2 H), 7.65 - 7.67 (m, 2 H), 7.59 (t, J=7.3 Hz, 1 H), $7.53-7.55$ (m, 2 H), 7.44 (t, J=7.8 Hz, 2 H), 5.69 (ddd, J=17.3, 10.7, 5.6 Hz, 1 H), 5.61 (t, J=6.1 Hz, 1 H), 5.30 (dd, J=10.5, 1.2 Hz, 1 H), 5.20 (dd, J=17.3, 1.2 Hz, 1 H), $4.72-4.84(m, 1$ H), 4.45 (dd, J=11.8, 9.0 Hz, 1 H), 4.35 (dd, J=11.8, $5.4 \mathrm{~Hz}, 1 \mathrm{H}$ ), 3.97 (ddd, J=18.7, 2.2, $2.1 \mathrm{~Hz}, 1 \mathrm{H}), 3.63-3.77(\mathrm{~m}, 3 \mathrm{H}) ;{ }^{13} \mathrm{C} N M R$ $(100 \mathrm{MHz}, \mathrm{CDCl} 3) \delta 166.4,139.7,134.1,134.1,133.8,133.2,132.5,131.8$ $131.5,129.8,129.5,129,128.8,128.6,128,125.7,120.4,80.6,78.7,63.3,58.4$, 33.6, 33.5; HRMS (ES+) $\mathrm{m} / \mathrm{z} 679.0542$ [calc'd for $\mathrm{C}_{27} \mathrm{H}_{28} \mathrm{BrN}_{4} \mathrm{O}_{8} \mathrm{~S}_{2}(\mathrm{M}+\mathrm{NH} 4)$ 679.0532].

These intermediate nosylates were then elaborated into 3-mers 12-14 following the above procedure for Mitsunobu oligomerization. 
Spectral data for N-Boc-ene-yne-ene (S,R)-12.

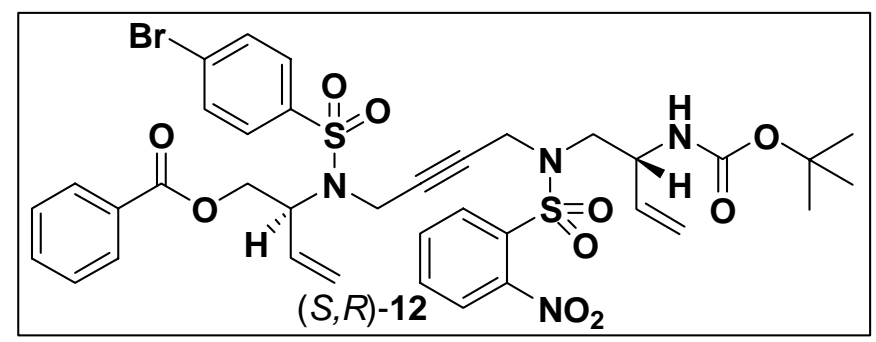

N-Boc-ene-yne-ene $(\boldsymbol{S}, \boldsymbol{R})-12$. Isolated as a pale yellow oil (121 mg, 93\% yield) $[\alpha]_{D}^{25}-18.2$ (c 0.11, $\mathrm{CH}_{2} \mathrm{Cl}_{2}$ ); IR (thin film/NaCl) 3399 (bs), 2925 (w), 2360 (w), 1707 (s), 1573 (w), 1544 (s), $1452(w), 1355$ (m), $1270(\mathrm{~s}), 1221(\mathrm{~m}), 1160$ (s), $1111(\mathrm{~m}), 1069(\mathrm{~m}), 1025(\mathrm{w}), 919(\mathrm{w}), 771(\mathrm{~m}), 736(\mathrm{~m}), 713(\mathrm{~s}) \mathrm{cm}^{-1} ; 1 \mathrm{H}$ NMR (400 MHz, CDCl3) $\delta$ ppm 7.95 - $8.02(\mathrm{~m}, 1 \mathrm{H}), 7.84$ - 7.90 (m, 2 H), 7.63 - 7.73 (m, 5 H), 7.51 - $7.59(m, 3 H), 7.42(t, J=7.9$ Hz, 2 H), 5.65 - $5.80(m, 2$ H), 5.16 5.31 (m, 4 H), 4.77 (q, J=6.3 Hz, 2 H), 4.43 (d, J=7.3 Hz, 2 H), 4.37 (br. s., 1 H), 3.88 - $4.25(\mathrm{~m}, 4 \mathrm{H}), 3.29-3.49(\mathrm{~m}, 2 \mathrm{H}), 1.41(\mathrm{~s}, 9 \mathrm{H}) ;{ }^{13} \mathrm{C} \mathrm{NMR}(100 \mathrm{MHz}$, CDCl3) $\delta 166.2,155.7,148.3,139.6,135.4,134,133.7,133.2,132.6,132.3$, $131.9,130.9,129.8,129.5,129,128.8,128.1,124.6,120.4,117.1,81.7,80$, 77.8, 63.3, 58.5, 50.2, 50.1, 37.1, 33.7, 28.6; HRMS (ES+) $\mathrm{m} / \mathrm{z} 831.1351$ [calc'd for $\mathrm{C}_{36} \mathrm{H}_{40} \mathrm{BrN}_{4} \mathrm{O}_{10} \mathrm{~S}_{2}(\mathrm{M}+\mathrm{H})$ 831.1369].

\section{Spectral data for N-Boc-ene-yne-ene (S,S)-12.}

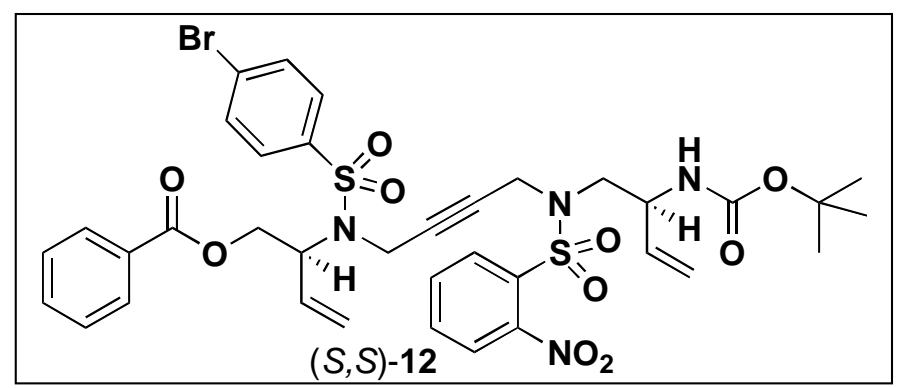


N-Boc-ene-yne-ene $(\mathbf{S}, \mathbf{S})-12$. Isolated as a pale yellow oil (108 mg, 73\% yield), $[\alpha]_{D}^{25}+19.6$ (c 0.05, $\mathrm{CH}_{2} \mathrm{Cl}_{2}$ ); IR (thin film/NaCl) 3399 (bs), 2978 (w), 2360 (w), 1709 (s), 1574 (w), 1544 (s), 1452 (w), 1354 (m), 1270 (s), 1161 (s), $1112(\mathrm{~m})$, $1069(\mathrm{~m}), 1026(\mathrm{w}), 922(\mathrm{w}), 774(\mathrm{~m}), 737(\mathrm{~m}), 713(\mathrm{~s}) \mathrm{cm}^{-1}$; 1H NMR $(600 \mathrm{MHz}$, CDCl3) $\delta$ ppm $7.99(\mathrm{dd}, J=7.5,1.3 \mathrm{~Hz}, 1 \mathrm{H}), 7.86(\mathrm{~d}, J=7.3 \mathrm{~Hz}, 2 \mathrm{H}), 7.63-7.72$ (m, 5 H), 7.52 - $7.59(m, 3 H), 7.42(t, J=7.7$ Hz, 2 H), 5.65 - $5.77(m, 2 H), 5.16$ 5.30 (m, 4 H), 4.72 - 4.85 (m, 2 H), 4.38 - 4.46 (m, 2 H), 4.36 (br. s., 1 H), 3.91 $4.19(\mathrm{~m}, 4 \mathrm{H}), 3.39$ - $3.49(\mathrm{~m}, 1 \mathrm{H}), 3.31$ (dd, J=14.6, $4.8 \mathrm{~Hz}, 1 \mathrm{H}), 1.41(\mathrm{~s}, 9 \mathrm{H})$; ${ }^{13} \mathrm{C}$ NMR $(100 \mathrm{MHz}, \mathrm{CDCl} 3) \delta 166.2,155.7,148.3,139.6,135.4,134.1,133.7$, 133.2, 132.6, 132.2, 131.9, 131, 129.8, 129.5, 129, 128.8, 128.1, 124.6, 120.4, 117.1, 81.7, 80, 77.8, 63.3, 58.5, 50.3, 50.2, 37.2, 33.7, 28.6; HRMS (ES+) $\mathrm{m} / \mathrm{z}$ 831.1334 [calc'd for $\mathrm{C}_{36} \mathrm{H}_{40} \mathrm{BrN}_{4} \mathrm{O}_{10} \mathrm{~S}_{2}(\mathrm{M}+\mathrm{H})$ 831.1369].

\section{Spectral data for (rac)-N-Boc-yne-yne-ene 13.}

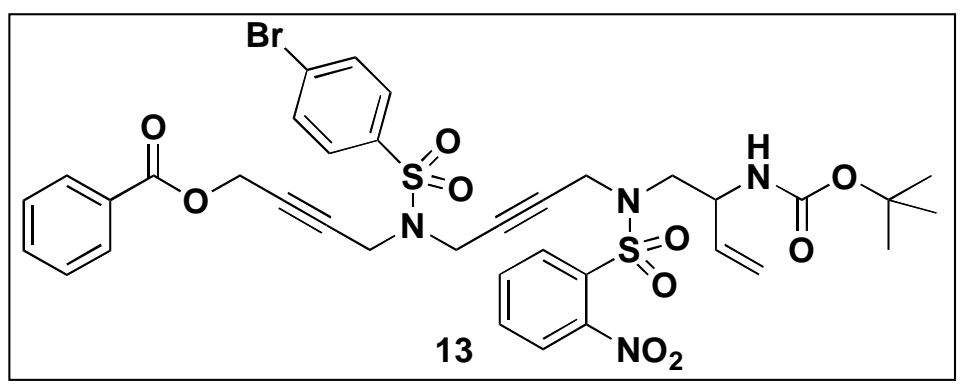

(rac)-N-Boc-yne-yne-ene 13. Isolated as a pale yellow oil (414 mg, 94\% yield); IR (thin film/NaCl) 3398 (bs), 2978 (w), 1708 (w), 1574 (w), 1544 (m), 1452 (w), $1356(\mathrm{~m}), 1271(\mathrm{~s}), 1163(\mathrm{~s}), 1092(\mathrm{~m}), 1069(\mathrm{~m}), 1009(\mathrm{w}), 906(\mathrm{w}), 760(\mathrm{~m})$, $714(\mathrm{~m}) \mathrm{cm}^{-1} ; 1 \mathrm{H} \mathrm{NMR}(600 \mathrm{MHz}, \mathrm{CDCl} 3) \delta \mathrm{ppm} 8.03(\mathrm{~d}, J=8.1 \mathrm{~Hz}, 2 \mathrm{H}), 7.97$ $8.02(m, 1 \mathrm{H}), 7.69-7.77(\mathrm{~m}, 2 \mathrm{H}), 7.60$ - $7.67(\mathrm{~m}, 6 \mathrm{H}), 7.48$ (t, J=7.7 Hz, 2 H), 
5.74 (ddd, J=17.0, 10.8, $5.5 \mathrm{~Hz}, 1 \mathrm{H}$ ), 5.28 (d, J=17.2 Hz, 1 H), 5.21 (d, J=10.3 Hz, 1 H), 4.71 (s, 3 H), 4.38 (br. s., 1 H), 4.26 (d, J=19.0 Hz, 1 H), 4.15 (d, J=19.0 Hz, 1 H), 4.05 (s, 2 H), 3.95 (s, 2 H), 3.47 (dd, J=13.2, 9.9 Hz, 1 H), 3.34 (dd, $J=14.1,5.3 \mathrm{~Hz}, 1 \mathrm{H}), 1.42(\mathrm{~s}, 9 \mathrm{H}) ;{ }^{13} \mathrm{C}$ NMR $(100 \mathrm{MHz}, \mathrm{CDCl} 3) \delta$ 165.9, 148.3, $137.4,135.2,134.3,133.8,132.9,132.6,132.1,131,130,129.4,128.8,128.5$, 124.6, 117.3, 80.8, 80.2, 79, 78.8, 77.5, 52.4, 50.3, 50, 37, 36.7, 28.6; HRMS (ES+) $m / z$ 829.1179 [calc'd for $\mathrm{C}_{36} \mathrm{H}_{38} \mathrm{BrN}_{4} \mathrm{O}_{10} \mathrm{~S}_{2}(\mathrm{M}+\mathrm{H})$ 829.1212].

\section{Spectral data for bicycle $(S, R)-14$.}

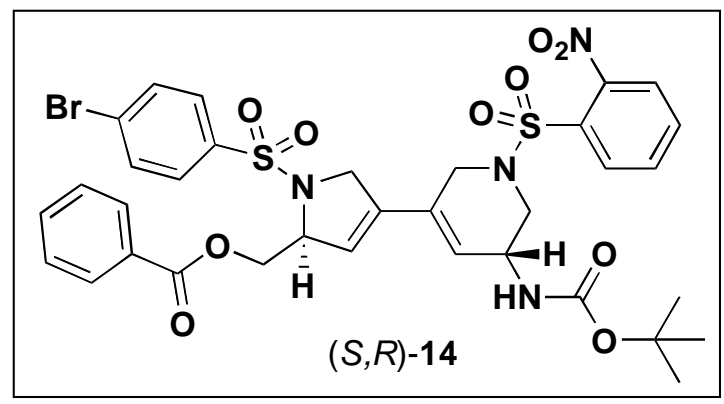

Bicycle $(\boldsymbol{S}, \boldsymbol{R})-14$. Isolated after $15 \mathrm{~h}$ reaction time as a colorless oil $(13 \mathrm{mg}, 57 \%$ yield), $[\alpha]_{D}^{25}+40.2\left(\mathrm{c} 0.48, \mathrm{CH}_{2} \mathrm{Cl}_{2}\right.$ ); IR (thin film/ $\mathrm{NaCl}$ ) 3365 (bs), 2975 (w), 2927 (w), 1706 (s), 1543 (m), 1504 (s), 1452 (w), 1355 (s), 1269 (s), 1160 (s), 1092 (s), 1067 (s), $1026(w), 1009$ (w), $973(w), 851(w), 826(w), 739(m), 712(m s)$ $\mathrm{cm}^{-1}$; $1 \mathrm{H}$ NMR $(500 \mathrm{MHz}, \mathrm{CDCl} 3) \delta \mathrm{ppm} 7.99$ (t, J=8.3 Hz, $3 \mathrm{H}$ ), 7.83 (d, J=8.3 Hz, 2 H), $7.72-7.80$ (m, 2 H), 7.66 (t, J=8.8 Hz, 3 H), 7.58 (t, J=7.3 Hz, 1 H), 7.44 (t, J=7.6 Hz, 2 H), 5.28 (d, J=9.3 Hz, 1 H), 4.94 - 5.08 (m, 2 H), 4.85 (br. s., $1 \mathrm{H}), 4.66(\mathrm{~d}, J=8.8 \mathrm{~Hz}, 1 \mathrm{H}), 4.60$ (d, J=13.7 Hz, $1 \mathrm{H}$ ), 4.50 (br. s., $1 \mathrm{H}$ ), 4.37 (br. s., $1 \mathrm{H}), 4.33$ (d, J=14.6 Hz, 1 H), 3.88 (d, J=12.7 Hz, 1 H), 3.71 (d, J=14.6 Hz, 1 H), 3.41 (d, J=13.7 Hz, 1 H), 3.13 (d, J=12.7 Hz, 1 H), 3.09 (s, 3 H), $1.43(\mathrm{~s}, 9 \mathrm{H})$; 
${ }^{13} \mathrm{C}$ NMR $(100 \mathrm{MHz}, \mathrm{CDCl} 3) \delta 166.4,155,148.5,136.5,136.2,134.4,133.5$, 132.9, 132.1, 131.4, 130.2, 130, 129.8, 129.1, 128.7, 128.4, 125.1, 124.5, 122.6, 80.4, 66.4, 66.5, 55.1, 47.9, 44.8, 44.7, 28.8; HRMS (ES+) $\mathrm{m} / \mathrm{z} 820.1295$ [calc'd for $\left.\mathrm{C}_{34} \mathrm{H}_{39} \mathrm{BrN}_{5} \mathrm{O}_{10} \mathrm{~S}_{2}(\mathrm{M}+\mathrm{NH} 4) 820.1322\right]$.

\section{Spectral data for bicycle $(S, S)-14$.}

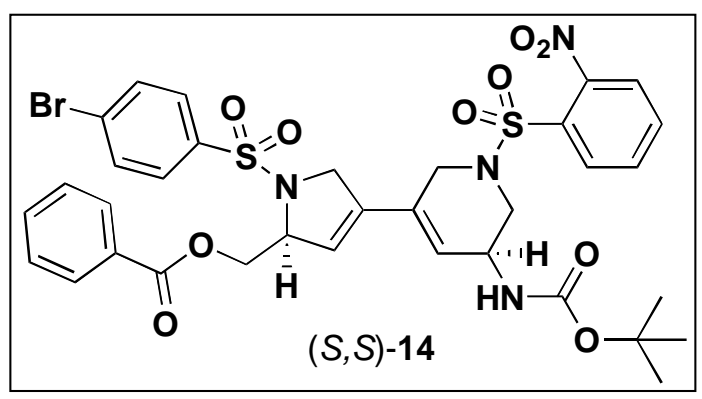

Bicycle (S,S)-14. Isolated after $24 \mathrm{~h}$ reaction time as a colorless oil (18 mg, $74 \%$ yield), $[\alpha]_{D}{ }^{25}-34.2$ (c 0.33, $\mathrm{CH}_{2} \mathrm{Cl}_{2}$ ); IR (thin film/NaCl) 3369 (bs), 2975 (w), 2927 (w), $1710(\mathrm{~s}), 1546(\mathrm{~m}), 1507(\mathrm{~m}), 1452(\mathrm{w}), 1367(\mathrm{~m}), 1272(\mathrm{~s}), 1164(\mathrm{~s}), 1095$ (s), $1068(\mathrm{~s}), 1027(w), 1007(w), 971(w), 852(w), 823(w), 741(\mathrm{~m}), 711(\mathrm{~m}) \mathrm{cm}^{-}$ 1; $1 \mathrm{H} \mathrm{NMR} \mathrm{(400} \mathrm{MHz,} \mathrm{CDCl3)} \delta$ ppm 7.99 (dd, J=7.6, 1.7 Hz, 1 H), 7.92 (d, J=7.6 Hz, 2 H), $7.54-7.75$ (m, 8 H), 7.41 (t, J=7.7 Hz, 2 H), 5.70 (d, J=1.5 Hz, 1 H), $5.61(\mathrm{~d}, J=4.4 \mathrm{~Hz}, 1 \mathrm{H}), 4.88$ (br. s., $1 \mathrm{H}), 4.78$ (d, J=8.8 Hz, $1 \mathrm{H}$ ), 4.70 (dd, $J=11.2,3.7 \mathrm{~Hz}, 1 \mathrm{H}), 4.40(\mathrm{dd}, J=11.2,5.6 \mathrm{~Hz}, 1 \mathrm{H}), 4.22-4.33(\mathrm{~m}, 3 \mathrm{H}), 4.13(\mathrm{~d}$, $J=16.4 \mathrm{~Hz}, 1 \mathrm{H}), 3.59-3.72(\mathrm{~m}, 2 \mathrm{H}), 3.11(\mathrm{~d}, \mathrm{~J}=15.4 \mathrm{~Hz}, 1 \mathrm{H}), 1.42(\mathrm{~s}, 9 \mathrm{H}) ;{ }^{13} \mathrm{C}$ NMR $(125 \mathrm{MHz}, \mathrm{CDCl} 3) \delta 166.3,155,148.5,136.6,136.3,134.3,133.5,133$, 132.9, 132.1, 131.3, 130.4, 129.8, 129.8, 129, 128.7, 128.5, 124.6, 124.5, 122.7, 80.4, 66.7, 66.5, 55.1, 48.2, 44.7, 29.9, 28.6; HRMS (ES+) $\mathrm{m} / \mathrm{z} 820.1313$ [calc'd for $\mathrm{C}_{34} \mathrm{H}_{39} \mathrm{BrN}_{5} \mathrm{O}_{10} \mathrm{~S}_{2}(\mathrm{M}+\mathrm{NH} 4)$ 820.1322]. 


\section{Preparation of tricyclic diene 15.}

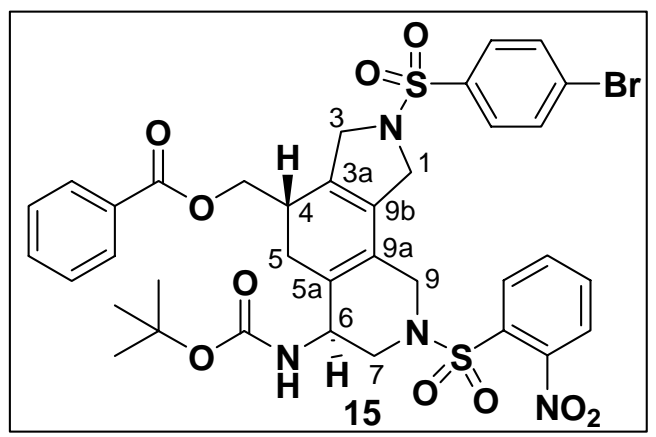

Tricyclic diene 15. Consistent with the metathesis procedure described above, a solution of yne-yne-ene $13(75 \mathrm{mg}, 0.092 \mathrm{mmol}, 1.0$ equiv) and $\left[\mathrm{RuCl}_{2}(=\mathrm{CHPh})\left(\mathrm{PCy}_{3}\right)_{2}\right](0.05$ equiv) in benzene $(0.03 \mathrm{M})$ was placed under ethylene atmosphere by two freeze-pump-thaw cycles. This mixture was heated under reflux for 22 hours, after which time a white precipitate formed in the flask. The solution was cooled to room temperature and filtered over a cotton plug, and the precipitate was recovered. Rotary evaporation of the filtrate followed by chromatography on silica gel (gradient elution, $\mathrm{CH}_{3} \mathrm{CN}$ in $\mathrm{CH}_{2} \mathrm{Cl}_{2}$ ) 15 as an amorphous white powder (42 mg, 56\% yield); IR (thin film/NaCl) 3367 (bs), 2925 (w), 1708 (s), 1573 (s), 1544 (s), 1507 (m), 1471 (w), 1350 (s), 1272 (s), 1166 (s), $1100(\mathrm{~m}), 1068(\mathrm{~m}), 1008(\mathrm{w}), 959(\mathrm{w}), 778(\mathrm{w}), 741(\mathrm{~m}), 713(\mathrm{~m}) \mathrm{cm}^{-1} ; 1 \mathrm{H}$ NMR (400 MHz, CDCl3) $\delta$ ppm 8.00 (dd, J=7.4, $1.6 \mathrm{~Hz}, 1 \mathrm{H}), 7.92(\mathrm{~d}, J=7.1 \mathrm{~Hz}, 2$ H), $7.69-7.80(\mathrm{~m}, 2 \mathrm{H}), 7.53-7.69(\mathrm{~m}, 6 \mathrm{H}), 7.44$ (t, J=7.7 Hz, $2 \mathrm{H}), 4.77$ (d, $J=9.5 \mathrm{~Hz}, 1 \mathrm{H}), 4.29-4.39(\mathrm{~m}, 1 \mathrm{H}), 4.06-4.29(\mathrm{~m}, 6 \mathrm{H}), 3.94(\mathrm{~d}, J=17.3 \mathrm{~Hz}, 1$ H), 3.75 (d, J=12.7 Hz, 1 H), 3.47 (d, J=14.6 Hz, 1 H), 3.02 (dd, J=13.1, $3.1 \mathrm{~Hz}, 1$ H), 2.81 (br. s., 1 H), 2.59 (dd, J=17.0, 8.9 Hz, 1 H), 2.23 (dd, J=17.3, 9.8 Hz, 1 $\mathrm{H}), 1.40(\mathrm{~s}, 9 \mathrm{H}) ;{ }^{13} \mathrm{C} \mathrm{NMR}(100 \mathrm{MHz}, \mathrm{CDCl} 3) \delta 166.3,155.6,148.5,136.1$, 
134.4, 133.6, 132.8, 132.1, 131.6, 131.5, 131.3, 129.8, 129.1, 129, 128.8, 128.3,

$128.2,124.5,123.7,80.4,65.3,55.7,53.2,48.5,48.1,44.4,32.9,29.4,28.5$;

HRMS (ES+) $m / z$ 846.1478 [calc'd for $\mathrm{C}_{36} \mathrm{H}_{41} \mathrm{BrN}_{5} \mathrm{O}_{10} \mathrm{~S}_{2}(\mathrm{M}+\mathrm{NH} 4)$ 846.1456].

Table 1. NMR data for 15 (600 MHz for ${ }^{1} \mathrm{H}, 151 \mathrm{MHz}$ for $\left.{ }^{13} \mathrm{C}, \mathrm{CDCl} 3\right)$.

\begin{tabular}{|c|c|c|c|}
\hline carbon & $\delta\left({ }^{13} \mathrm{C}\right) / \mathrm{ppm}$ & proton & $\delta\left({ }^{1} \mathrm{H}\right) / \mathrm{ppm}$ \\
\hline $\mathrm{C}-1$ & 53.16 & $1-\mathrm{H}_{\mathrm{a}}$ & 4.12 \\
\hline & & $1-\mathrm{H}_{\mathrm{b}}$ & 4.16 \\
\hline $\mathrm{C}-2$ & 55.70 & $3-\mathrm{H}_{\mathrm{a}}$ & 4.16 \\
\hline & & $3-\mathrm{H}_{\mathrm{b}}$ & 4.33 \\
\hline $\mathrm{C}-3 \mathrm{a}$ & 131.47 & & \\
\hline $\mathrm{C}-4$ & 32.91 & $4-\mathrm{H}$ & 2.80 \\
\hline $4-\mathrm{CH}_{2}-\mathrm{OBz}$ & 65.24 & $\mathrm{CH}_{\mathrm{a}} \mathrm{H}_{\mathrm{b}}-\mathrm{OBz}$ & 4.19 \\
\hline & & $\mathrm{CH}_{\mathrm{a}} \mathrm{H}_{\mathrm{b}}-\mathrm{OBz}$ & 4.23 \\
\hline $\mathrm{C}-5$ & 29.34 & $5-\mathrm{H}_{\mathrm{a}}$ & 2.21 \\
\hline & & $5-\mathrm{H}_{\mathrm{b}}$ & 2.58 \\
\hline $\mathrm{C}-5 \mathrm{a}$ & 128.31 & & \\
\hline $\mathrm{C}-6$ & 48.10 & $6-\mathrm{H}$ & 4.13 \\
\hline & & $6-\mathrm{NH}$ & 4.78 \\
\hline $\mathrm{C}-7$ & 48.52 & $7-\mathrm{Ha}$ & 3.02 \\
\hline & & $7-\mathrm{Hb}$ & 3.74 \\
\hline $\mathrm{C}-9$ & 44.36 & $9-\mathrm{H}_{\mathrm{a}}$ & 3.46 \\
\hline & & $9-\mathrm{H}_{\mathrm{b}}$ & 3.94 \\
\hline $\mathrm{C}-9 \mathrm{a}$ & 123.62 & & \\
\hline $\mathrm{C}-9 \mathrm{~b}$ & 129.01 & & \\
\hline \multicolumn{2}{|r}{} & &
\end{tabular}

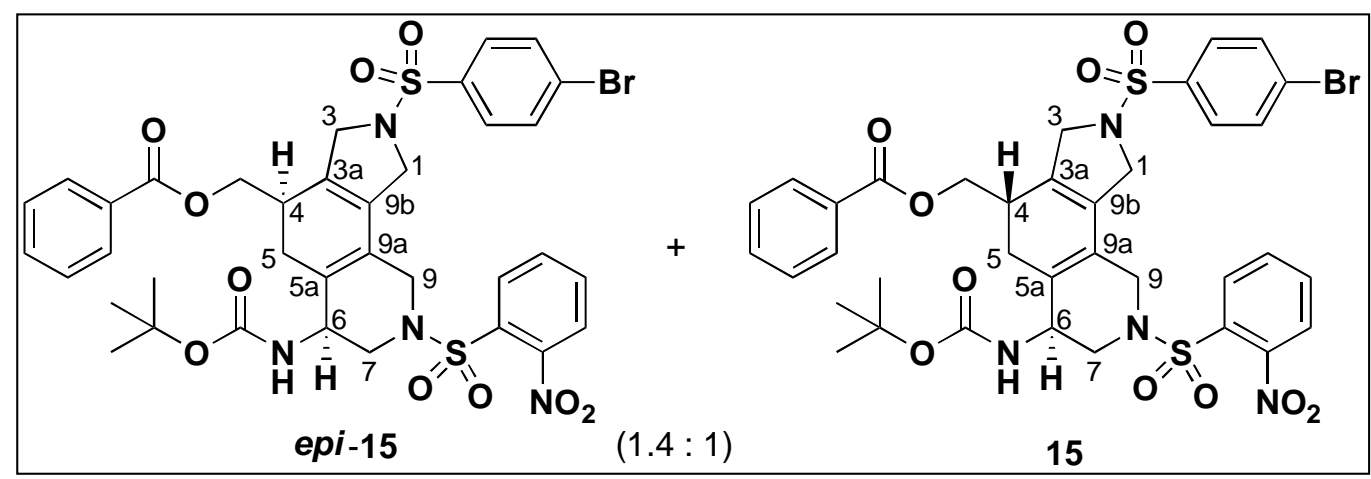

The precipitate from the above reaction was then dissolved in $\mathrm{CHCl}_{3}$, and the solvent was evaporated under reduced pressure to provide a mixture of 15 and 
its diastereomer (28 $\mathrm{mg}, 37 \%$ yield, 1:1.4 ratio), which was resistant to further chromatographic purification. This mixture yielded the following spectral properties: $1 \mathrm{H} \mathrm{NMR}(500 \mathrm{MHz}, \mathrm{CDCl} 3) \delta \mathrm{ppm} 8.02(\mathrm{t}, \mathrm{J}=8.3 \mathrm{~Hz}, 2.4 \mathrm{H}), 7.94(\mathrm{t}$, J=8.1 Hz, $4.8 \mathrm{H}), 7.70-7.82(\mathrm{~m}, 4.8 \mathrm{H}), 7.54-7.71(\mathrm{~m}, 14.4 \mathrm{H}), 7.39-7.52(\mathrm{~m}$, $4.8 \mathrm{H}), 4.83(\mathrm{~d}, J=9.3 \mathrm{~Hz}, 1.4 \mathrm{H}), 4.77$ (d, J=8.8 Hz, $1.0 \mathrm{H}), 4.34$ (d, J=11.7 Hz, $1.0 \mathrm{H}), 4.06$ - $4.30(\mathrm{~m}, 14.4 \mathrm{H}), 3.96(\mathrm{t}, \mathrm{J}=15.1 \mathrm{~Hz}, 2.4 \mathrm{H}), 3.69$ - $3.80(\mathrm{~m}, 2.4 \mathrm{H})$, $3.57(\mathrm{~d}, \mathrm{~J}=16.1 \mathrm{~Hz}, 1.4 \mathrm{H}), 3.47$ (d, J=16.6 Hz, 1.0 H), 3.12 (d, J=12.7 Hz, $1.4 \mathrm{H}$ ), 3.03 (d, J=12.7 Hz, $1.0 \mathrm{H}$ ), 2.81 (br. s., $2.4 \mathrm{H}), 2.53$ - 2.66 (m, $1.0 \mathrm{H}), 2.41$ (d, J=5.9 Hz, $2.8 \mathrm{H}), 2.15-2.29(\mathrm{~m}, 1.0 \mathrm{H}), 1.78-1.99(\mathrm{~m}, 1.4 \mathrm{H}), 1.40(\mathrm{~s}, 12.6 \mathrm{H})$, 1.35 (s, $9.0 \mathrm{H}) ;{ }^{13} \mathrm{C}$ NMR $(100 \mathrm{MHz}, \mathrm{CDCl} 3) \delta 166.5,166.3,155.7,155.6,148.5$, $136.1,134.4,133.7,133.6,132.9,132.1,131.6,131.5,131.4,129.8,129.2$, $129.1,129,128.9,128.8,128.3,128.2,128.1,124.6,123.7,123.6,80.4,80.2$, 65.3, 64.8, 55.7, 53.2, 48.5, 48.1, 47.8, 44.4, 32.9, 32.4, 29.4, 28.8, 28.5, 28.5.; HRMS (ES+) $\mathrm{m} / \mathrm{z} 844.1351$ [calc'd for $\mathrm{C}_{36} \mathrm{H}_{41} \mathrm{BrN}_{5} \mathrm{O}_{10} \mathrm{~S}_{2}(\mathrm{M}+\mathrm{NH} 4)$ 844.1322]

Stereochemical assignments of epi-15 and 15 are based on the observation of NOE's between the BocN-H proton and $4-\mathrm{CH}_{\mathrm{a}} H_{\mathrm{b}}-\mathrm{OBz}$ for epi-15 and observation of NOE's between $6-\mathrm{H}$ and $5-\mathrm{H}_{a}, 5-\mathrm{H}_{\mathrm{a}}$ and $4-\mathrm{CH}_{\mathrm{a}} \mathrm{H}_{\mathrm{b}}-\mathrm{OBz}$, as well as $5-\mathrm{H}_{\mathrm{b}}$ and $6-\mathrm{NH}$ for 15. Full NMR-spectroscopic assignments for the protons and carbons representing the tricyclic cores in $\mathbf{1 5}$ and epi-15 are given in Tables 1 and 2, respectively.

Table 2. NMR data for epi-15 (600 MHz for ${ }^{1} \mathrm{H}, 151 \mathrm{MHz}$ for $\left.{ }^{13} \mathrm{C}, \mathrm{CDCl} 3\right)$.

\begin{tabular}{|c|c|c|c|}
\hline carbon & $\delta\left({ }^{13} \mathrm{C}\right) / \mathrm{ppm}$ & proton & $\delta\left({ }^{1} \mathrm{H}\right) / \mathrm{ppm}$ \\
\hline $\mathrm{C}-1$ & 53.16 & $1-\mathrm{H}_{\mathrm{a}}$ & 4.16 \\
\hline & & $1-\mathrm{H}_{\mathrm{b}}$ & 4.16 \\
\hline
\end{tabular}




\begin{tabular}{|c|c|c|c|}
\hline $\mathrm{C}-3$ & 55.70 & $3-\mathrm{H}_{\mathrm{a}}$ & 4.11 \\
\hline & & $3-\mathrm{H}_{\mathrm{b}}$ & 4.24 \\
\hline $\mathrm{C}-3 \mathrm{a}$ & 131.36 & & \\
\hline $\mathrm{C}-4$ & 32.37 & $4-\mathrm{H}$ & 2.80 \\
\hline $4-\mathrm{CH}_{2}-\mathrm{OBz}$ & 64.78 & $\mathrm{CH}_{\mathrm{a}} \mathrm{H}_{\mathrm{b}}-\mathrm{OBz}$ & 4.11 \\
\hline & & $\mathrm{CH}_{\mathrm{a}} \mathrm{H}_{\mathrm{b}}-\mathrm{OBz}$ & 4.22 \\
\hline $\mathrm{C}-5$ & 28.80 & $5-\mathrm{H}_{\mathrm{a}}$ & 2.40 \\
\hline & & $5-\mathrm{H}_{\mathrm{b}}$ & 2.40 \\
\hline $\mathrm{C}-5 \mathrm{a}$ & 128.08 & & \\
\hline $\mathrm{C}-6$ & 47.73 & $6-\mathrm{H}$ & 4.14 \\
\hline & & $6-\mathrm{NH}$ & 4.83 \\
\hline $\mathrm{C}-7$ & 48.47 & $7-\mathrm{Ha}$ & 3.11 \\
\hline & & $7-\mathrm{Hb}$ & 3.73 \\
\hline $\mathrm{C}-9$ & 44.36 & $9-\mathrm{H}_{\mathrm{a}}$ & 3.56 \\
\hline & & $9-\mathrm{H}_{\mathrm{b}}$ & 3.97 \\
\hline $\mathrm{C}-9 \mathrm{a}$ & 123.50 & & \\
\hline $\mathrm{C}-9 \mathrm{~b}$ & 129.19 & & \\
\hline
\end{tabular}

\section{Preparation of Alcohol A7.}

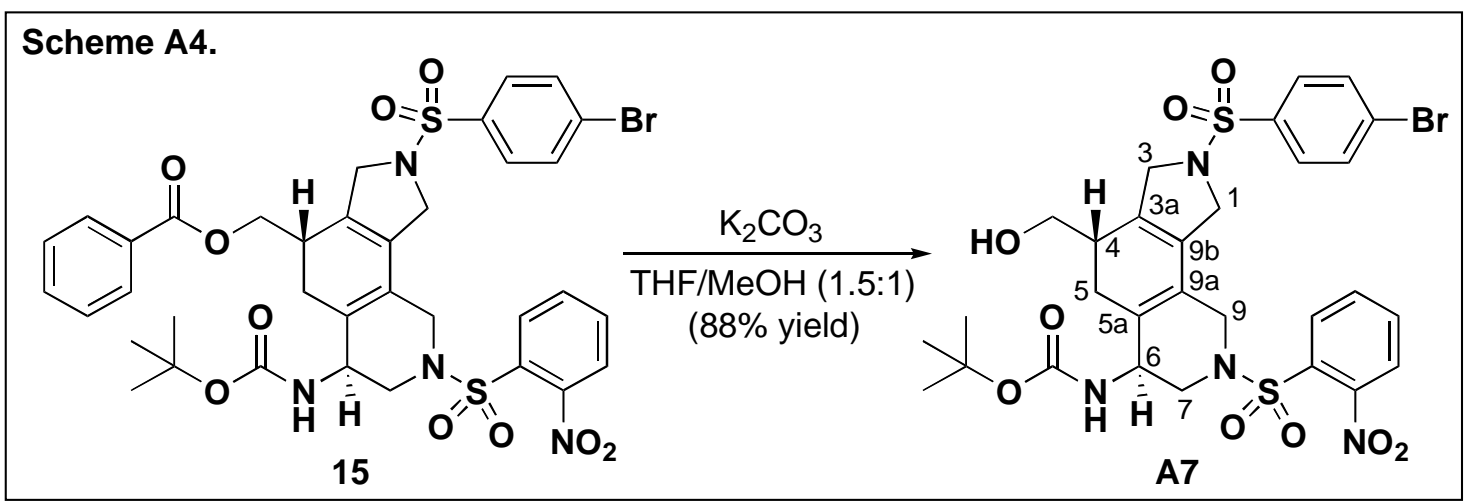

Alcohol A7. To a solution of 15 (19 mg, $0.023 \mathrm{mmol}, 1.0$ equiv) in THF (0.15 $\mathrm{mL}$ ) was added $\mathrm{MeOH}(0.23 \mathrm{~mL})$ and anhydrous potassium carbonate (16 mg, $0.117 \mathrm{mmol}, 5.0$ equiv). This mixture was allowed to stir for 30 minutes at which time it was diluted with saturated aqueous ammonium chloride $(1 \mathrm{~mL})$, and extracted with EtOAc $(6 \times 2 \mathrm{~mL})$. The combined organic phases were dried over sodium sulfate, filtered, evaporated under reduced pressure. Flash 
chromatography on silica gel yielded A4 as a pale yellow oil $(14.6 \mathrm{mg}, 88 \%$ yield); $1 \mathrm{H} \mathrm{NMR} \mathrm{(600} \mathrm{MHz,} \mathrm{CDCl3)} \delta \mathrm{ppm} 7.91(\mathrm{~d}, \mathrm{~J}=6.7 \mathrm{~Hz}, 1 \mathrm{H}), 7.53$ - $7.68(\mathrm{~m}$, $7 \mathrm{H}), 4.71(\mathrm{~d}, J=9.1 \mathrm{~Hz}, 1 \mathrm{H}), 4.19-4.30(\mathrm{~m}, 1 \mathrm{H}), 3.95-4.10(\mathrm{~m}, 4 \mathrm{H}), 3.86(\mathrm{~d}$, J=16.4 Hz, $1 \mathrm{H}$ ), 3.66 (d, J=12.9 Hz, $1 \mathrm{H}), 3.38-3.51(\mathrm{~m}, 3 \mathrm{H}), 2.96$ (d, J=12.3 Hz, 1 H), 2.45 (br. s., $1 \mathrm{H}$ ), 2.34 (dd, J=17.0, $9.1 \mathrm{~Hz}, 1 \mathrm{H}), 2.00-2.11(\mathrm{~m}, 1 \mathrm{H})$, 1.51 (br. s., $1 \mathrm{H}$ ), 1.30 (s, $9 \mathrm{H}$ ); ${ }^{13} \mathrm{C}$ NMR (100 MHz, PhH-d6) $\delta$ 155.7, 148.5, $136.3,134.3,132.9,132.1,131.5,131.4,129.1,128.3,128.2,128.1,124.5$, $123.5,80.3,63.8,55.8,53,48.6,48.1,44.4,35.9,29.9,28.7,28.5$.

Stereochemical assignment of $\mathbf{A} 7$ was based on the observation of NOE's between $6-\mathrm{H}$ and $5-\mathrm{H}_{a}, 5-\mathrm{H}_{a}$ and $4-\mathrm{CH}_{a} \mathrm{H}_{b}-\mathrm{OH}$, as well as $5-\mathrm{H}_{b}$ and $6-\mathrm{NH}$. Full NMR-spectroscopic assignments for the protons and carbons representing the tricyclic core of $\mathbf{A} \mathbf{7}$ are given in Table 3 .

Table 3. NMR data for $\mathbf{A} 7\left(600 \mathrm{MHz}\right.$ for ${ }^{1} \mathrm{H}, 151 \mathrm{MHz}$ for $\left.{ }^{13} \mathrm{C}, \mathrm{CDCl}\right)$.

\begin{tabular}{|c|c|c|c|}
\hline carbon & $\delta\left({ }^{13} \mathrm{C}\right) / \mathrm{ppm}$ & proton & $\delta\left({ }^{1} \mathrm{H}\right) / \mathrm{ppm}$ \\
\hline $\mathrm{C}-1$ & 53.00 & $1-\mathrm{H}_{\mathrm{a}}$ & 4.12 \\
\hline & & $1-\mathrm{H}_{\mathrm{b}}$ & 4.12 \\
\hline $\mathrm{C}-3$ & 55.74 & $3-\mathrm{H}_{\mathrm{a}}$ & 4.11 \\
\hline & & $3-\mathrm{H}_{\mathrm{b}}$ & 4.34 \\
\hline $\mathrm{C}-3 \mathrm{a}$ & 132.76 & & \\
\hline $\mathrm{C}-4$ & 35.90 & $4-\mathrm{H}$ & 2.55 \\
\hline $4-\mathrm{CH}_{2}-\mathrm{OH}$ & 63.79 & $\mathrm{CH}_{\mathrm{a}} \mathrm{H}_{\mathrm{b}}-\mathrm{OH}$ & 3.54 \\
\hline & & $\mathrm{CH}_{\mathrm{a}} \mathrm{H}_{\mathrm{b}}-\mathrm{OH}$ & 3.56 \\
\hline $\mathrm{C}-5$ & 28.59 & $5-\mathrm{H}_{\mathrm{a}}$ & 2.14 \\
\hline & & $5-\mathrm{H}_{\mathrm{b}}$ & 2.44 \\
\hline $\mathrm{C}-5 \mathrm{a}$ & 128.31 & & \\
\hline $\mathrm{C}-6$ & 48.10 & $6-\mathrm{H}$ & 4.095 \\
\hline & & $6-\mathrm{NH}$ & 4.80 \\
\hline $\mathrm{C}-7$ & 48.52 & $7-\mathrm{Ha}$ & 3.06 \\
\hline & & $7-\mathrm{Hb}$ & 3.75 \\
\hline $\mathrm{C}-9$ & 44.40 & $9-\mathrm{H}_{\mathrm{a}}$ & 3.53 \\
\hline & & $9-\mathrm{H}_{\mathrm{b}}$ & 3.95 \\
\hline $\mathrm{C}-9 \mathrm{a}$ & 123.39 & & \\
\hline
\end{tabular}


General procedure for Diels-Alder cycloaddition reactions (Scheme 5). Into a solution of diene (1.0 equiv) in $\mathrm{CH}_{2} \mathrm{Cl}_{2}(0.1 \mathrm{M})$ cooled to $0{ }^{\circ} \mathrm{C}$ was titrated 4methyl-1,2,4-triazoline-3,5-dione (1.0 $\mathrm{M}$ solution in $\mathrm{CH}_{2} \mathrm{Cl}_{2}, 1.5-2.0$ equiv) dropwise by syringe until a red color persisted in solution. The mixture was then warmed to room temperature, the solvent evaporated, and the residue purified by chromatography on silica gel (gradient elution, $\mathrm{CH}_{3} \mathrm{CN}$ in $\mathrm{CH}_{2} \mathrm{Cl}_{2}$ ) to provide cycloadducts $\mathbf{1 8 - 2 2}$ in the yields indicated.

\section{Spectral data for (rac)-Tricyclic Cycloadduct 18.}

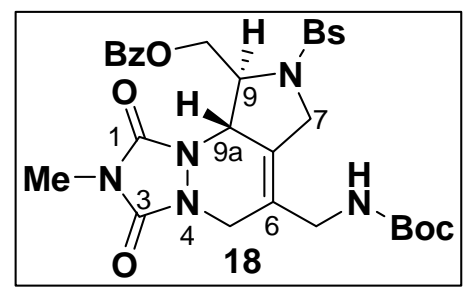

(rac)-Tricyclic Cycloadduct 18. Isolated as a colorless oil (17 mg, 94\% yield). Non-racemic material was carried through this sequence but the optical rotation was not obtained. For more complete characterization the racemic material was synthesized whose characterization data is shown here. IR (thin film/ $\mathrm{NaCl}$ ) 3386 (w), $2976(w), 1774(w), 1713(s), 1574(w), 1513(w), 1271(m), 1165(m), 748$ (w), $712\left(\right.$ w) $\mathrm{cm}^{-1} ; 1 \mathrm{H} \mathrm{NMR}(600 \mathrm{MHz}, \mathrm{CDCl} 3) \delta \mathrm{ppm} 7.90(\mathrm{~d}, J=7.3 \mathrm{~Hz}, 2 \mathrm{H})$, 7.68 (d, J=7.9 Hz, 2 H), 7.56 (d, J=7.9 Hz, 3 H), 7.42 (t, J=7.5 Hz, 2 H), 5.01 (d, $J=11.4 \mathrm{~Hz}, 1 \mathrm{H}), 4.87(\mathrm{~d}, J=11.4 \mathrm{~Hz}, 1 \mathrm{H}), 4.61-4.72(\mathrm{~m}, 2 \mathrm{H}), 4.55$ (d, J=14.4 Hz, 1 H), 4.34 (br. s., 1 H), 4.27 (d, J=16.4 Hz, 1 H), 3.82 - 3.94 (m, 2 H), 3.50 - 
$3.63(\mathrm{~m}, 2 \mathrm{H}), 3.09(\mathrm{~s}, 3 \mathrm{H}), 1.45(\mathrm{~s}, 9 \mathrm{H}) ;{ }^{13} \mathrm{C} \mathrm{NMR}(125 \mathrm{MHz}, \mathrm{CDCl} 3) \delta 166$, 155.9, 152.9, 137.8, 133.5, 132.7, 129.8, 129.1, 128.7, 128.5, 128.4, 124.6, 80.7, 65.8, 61.9, 58.8, 48.4, 43.5, 40.4, 28.6, 25.4; HRMS (ES+) $\mathrm{m} / \mathrm{z} 707.1475$ [calc'd for $\mathrm{C}_{29} \mathrm{H}_{32} \mathrm{BrN}_{5} \mathrm{O}_{8} \mathrm{~S}\left(\mathrm{M}+\mathrm{NH}_{4}\right)$ 707.1499].

Stereochemical assignment of $\mathbf{1 8}$ was based on the observation of strong NOE's between $9 \mathrm{a}-\mathrm{H}$ and $7-\mathrm{H}_{\mathrm{a}}(3.86 \mathrm{ppm}), 9 \mathrm{a}-\mathrm{H}$ and $9-\mathrm{CH}_{\mathrm{a}} H_{\mathrm{b}}-\mathrm{O}$, as well as between $7-\mathrm{H}_{a}$ and $9-\mathrm{CH}_{\mathrm{a}} \mathrm{H}_{\mathrm{b}}-\mathrm{O}$, suggesting that $9 \mathrm{a}-\mathrm{H}, 9-\mathrm{CH}_{2}$, and $7-\mathrm{H}_{\mathrm{a}}$ are situated on the same side of the tricyclus. In addition, strong NOE's were observed between $9-\mathrm{H}$ and $7-\mathrm{H}_{\mathrm{b}}$ (4.54 ppm), which further corroborated this assignment.

(rac)-Tricyclic Cycloadducts 19. A diastereomeric mixture of 19 (15 mg, 97\% yield) was isolated from the reaction and this was further purified by HPLC to provide the single diastereomers indicated.

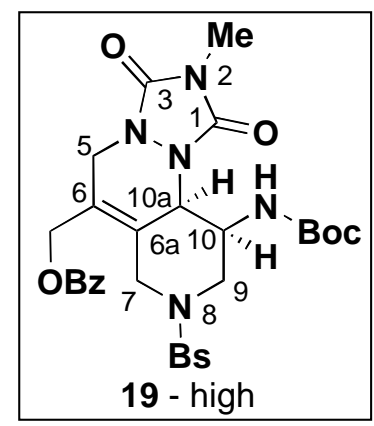

Higher Rf diastereomer: IR (thin film/NaCl) 2252 (w), 1769 (w), 1708 (s), 1502 (m), $1471(m), 1362(m), 1269(m), 1170(m), 1095(m), 937(w), 755(w), 733$ (w), 713 (w) $\mathrm{cm}^{-1}$; $1 \mathrm{H} \mathrm{NMR} \mathrm{(600} \mathrm{MHz,} \mathrm{CDCl3)} \delta \mathrm{ppm} 8.02$ (d, J=7.6 Hz, $2 \mathrm{H}$ ), $7.72(d, J=8.3 \mathrm{~Hz}, 2 \mathrm{H}), 7.63-7.69$ (m, 2 H), 7.60 (t, J=7.3 Hz, 1 H), 7.46 (dd, 2 H), $5.26(d, J=12.9 \mathrm{~Hz}, 1 \mathrm{H}), 4.86-5.01(\mathrm{~m}, 3 \mathrm{H}), 4.69(\mathrm{~d}, J=7.6 \mathrm{~Hz}, 1 \mathrm{H}), 4.36$ 
(d, J=16.4 Hz, 1 H), 4.28 (br. s., 1 H), 3.98 (d, J=16.1 Hz, 1 H), 3.85 (d, J=12.2 Hz, 1 H), 3.06 (s, 3 H), 2.90 (d, J=12.5 Hz, 1 H), 2.79 (d, J=12.2 Hz, 1 H), 1.36 (s, $9 \mathrm{H}) ;{ }^{13} \mathrm{C}$ NMR $(125 \mathrm{MHz}, \mathrm{CDCl} 3) \delta$ 166.2, 155.1, 154.3, 152.5, 134.7, 133.9, $133.1,130,129.3,129.1,128.9,128,124,80.6,61.6,56,50.5,47.6,46.7,45$, 28.4, 25.3; HRMS (ES+) $\mathrm{m} / z$ 707.1499 [calc'd for $\mathrm{C}_{29} \mathrm{H}_{32} \mathrm{BrN}_{5} \mathrm{O}_{8} \mathrm{~S}\left(\mathrm{M}+\mathrm{NH}_{4}\right)$ 707.1498].

Table 4. NMR data for 19 -high $\left(600 \mathrm{MHz}\right.$ for ${ }^{1} \mathrm{H}, 151 \mathrm{MHz}$ for $\left.{ }^{13} \mathrm{C}, \mathrm{CDCl}\right)$.

\begin{tabular}{|c|c|c|c|}
\hline carbon & $\delta\left({ }^{13} \mathrm{C}\right) / \mathrm{ppm}$ & proton & $\delta\left({ }^{1} \mathrm{H}\right) / \mathrm{ppm}$ \\
\hline $\mathrm{C}-1$ & 152.45 & & \\
\hline $2-\mathrm{CH}_{3}$ & 25.19 & $2-\mathrm{CH}_{3}$ & 3.06 \\
\hline C-3 & 154.09 & & \\
\hline \multirow[t]{2}{*}{ C-5 } & 44.86 & $5-\mathrm{H}_{\mathrm{a}}$ & 3.98 \\
\hline & & $5-\mathrm{H}_{b}$ & 4.36 \\
\hline C-6 & 127.96 & & \\
\hline \multirow{2}{*}{$6-\mathrm{CH}_{2}-\mathrm{OBz}$} & 61.55 & $\mathrm{CH}_{\mathrm{a}} \mathrm{H}_{\mathrm{b}}-\mathrm{OBz}$ & 4.91 \\
\hline & & $\mathrm{CH}_{\mathrm{a}} \mathrm{H}_{\mathrm{b}}-\mathrm{OBz}$ & 5.26 \\
\hline 6- $\mathrm{CH}_{2}-\mathrm{OBz}$ & $\begin{array}{c}166.16,133.86 \\
129.95,129.26 \\
128.80 \\
\end{array}$ & 6- $\mathrm{CH}_{2}-\mathrm{OBz}$ & $8.01,7.60,7.46$ \\
\hline C-6a & 123.97 & & \\
\hline \multirow[t]{2}{*}{ C-7 } & 47.52 & $7-\mathrm{H}_{\mathrm{a}}$ & 2.895 \\
\hline & & $7-\mathrm{H}_{\mathrm{b}}$ & 4.97 \\
\hline $8-B s$ & $\begin{array}{l}\text { 134.82, 133.06, } \\
\text { 129.32, 128.95, }\end{array}$ & $8-B s$ & $7.657,7.718$ \\
\hline \multirow[t]{2}{*}{ C-9 } & 50.42 & $9-\mathrm{H}_{\mathrm{a}}$ & 2.785 \\
\hline & & $9-\mathrm{H}_{\mathrm{b}}$ & 3.85 \\
\hline \multirow[t]{2}{*}{ C-10 } & 46.69 & $10-\mathrm{H}$ & 4.68 \\
\hline & & 10-NH & 4.93 \\
\hline $10-\mathrm{NH}-B o c$ & $\begin{array}{c}155.03,80.73 \\
28.47\end{array}$ & $10-\mathrm{NH}-B o c$ & 1.36 \\
\hline C-10a & 55.90 & $10 \mathrm{a}-\mathrm{H}$ & 4.28 \\
\hline
\end{tabular}




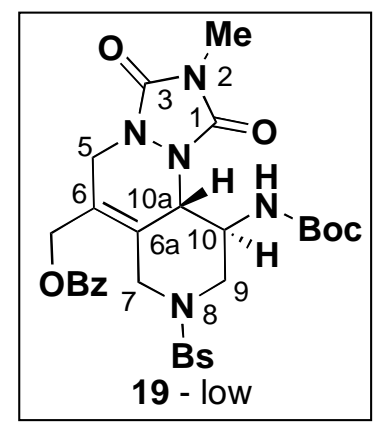

Lower Rf diastereomer: IR (thin film/NaCl) 3359 (w), 2978 (w), 1774 (w), 1708 (s), $1513(w), 1471(m), 1364(m), 1269(m), 1168(m), 1091(m), 934(w), 756$ (w), $736(w), 714(w) \mathrm{cm}^{-1}$; 1H NMR (500 MHz, CDCl3) $\delta$ ppm 8.00 (d, J=7.5 Hz, 2 H), 7.71 (s, 4 H), 7.60 (t, J=7.1 Hz, 1 H), 7.47 (t, J=7.6 Hz, 2 H), 5.43 (d, J=5.9 Hz, 1 H), 5.13 (d, J=13.2 Hz, 1 H), 4.85 (d, J=13.2 Hz, 1 H), 4.80 (d, J=13.2 Hz, 1 H), 4.23 - 4.37 (m, 2 H), $3.89-4.07(m, 3$ H), $3.17(d, J=12.2$ Hz, 1 H), 3.04 (s, 3 H), 2.65 (t, J=11.0 Hz, $1 \mathrm{H}), 1.41$ (s, $9 \mathrm{H}) ;{ }^{13} \mathrm{C} \mathrm{NMR} \mathrm{(125} \mathrm{MHz,} \mathrm{CDCl3)} \delta$ 166.2, $155.4,155.1,153,136.1,133.9,133,130,129.3,128.9,128.7,126.8,125.8$, 80.4, 61.4, 57.3, 53.1, 49.9, 47.2, 46.3, 28.6, 25.6; HRMS (ES+) $m / z$ 707.1487 [calc'd for $\mathrm{C}_{29} \mathrm{H}_{32} \mathrm{BrN}_{5} \mathrm{O}_{8} \mathrm{~S}\left(\mathrm{M}+\mathrm{NH}_{4}\right)$ 707.1499].

Table 5. NMR data for 19 -low (600 MHz for ${ }^{1} \mathrm{H}, 151 \mathrm{MHz}$ for $\left.{ }^{13} \mathrm{C}, \mathrm{CDCl} 3\right)$.

\begin{tabular}{|c|c|c|c|}
\hline carbon & $\delta\left({ }^{13} \mathrm{C}\right) / \mathrm{ppm}$ & proton & $\delta\left({ }^{1} \mathrm{H}\right) / \mathrm{ppm}$ \\
\hline $\mathrm{C}-1$ & 152.85 or 155.31 & & \\
\hline $2-\mathrm{CH}_{3}$ & 25.44 & $2-\mathrm{CH}_{3}$ & 3.04 \\
\hline $\mathrm{C}-3$ & 152.85 or 155.31 & & \\
\hline $\mathrm{C}-5$ & 46.73 & $5-\mathrm{H}_{\mathrm{a}}$ & 4.01 \\
\hline & & $5-\mathrm{H}_{\mathrm{b}}$ & 4.32 \\
\hline $\mathrm{C}-6$ & 125.66 or 126.71 & & \\
\hline $6-\mathrm{CH}_{2}-\mathrm{OBz}$ & 61.30 & $\mathrm{CH}_{\mathrm{a}} \mathrm{H}_{\mathrm{b}}-\mathrm{OBz}$ & 4.79 \\
\hline & & $\mathrm{CH}_{\mathrm{a}} \mathrm{H}_{\mathrm{b}}-\mathrm{OBz}$ & 5.11 \\
\hline $6-\mathrm{CH}_{2}-\mathrm{OBz}$ & $166.26,133.84$, & $6-\mathrm{CH}_{2}-\mathrm{OBz}$ & $7.99,7.60,7.45$ \\
& $129.99,129.25$, & & \\
\hline $\mathrm{C}-6 \mathrm{a}$ & 128.79 & & \\
\hline $\mathrm{C}-7$ & 125.66 or 126.71 & & \\
\hline
\end{tabular}




\begin{tabular}{|c|c|c|c|}
\hline & & $7-\mathrm{H}_{\mathrm{b}}$ & 4.85 \\
\hline $8-B s$ & $\begin{array}{c}136.14,132.94, \\
129.28,128.58\end{array}$ & $8-\mathrm{Bs}$ & $7.676,7.679$ \\
\hline $\mathrm{C}-9$ & 49.925 & $9-\mathrm{H}_{\mathrm{a}}$ & 2.655 \\
\hline & & $9-\mathrm{H}_{\mathrm{b}}$ & 3.973 \\
\hline $\mathrm{C}-10$ & 53.08 & $10-\mathrm{H}$ & 3.972 \\
\hline & & $10-\mathrm{NH}$ & 5.421 \\
\hline $10-\mathrm{NH}-$ Boc & $155.05,80.42$, & $10-\mathrm{NH}-\mathrm{Boc}$ & 1.40 \\
& 28.51 & $10 \mathrm{a}-\mathrm{H}$ & 4.30 \\
\hline $\mathrm{C}-10 \mathrm{a}$ & 57.23 & \multicolumn{2}{|l}{} \\
\hline
\end{tabular}

The relative configurations of 19-high and 19-low were assigned based on the observation of NOE's between $7-\mathrm{H}_{a}$ and $9-\mathrm{H}_{a}, 9-\mathrm{H}_{a}$ and $10 \mathrm{a}-\mathrm{H}$, as well as 10a- $\mathrm{H}$ and $7-\mathrm{H}_{\mathrm{a}}$ for $19-\mathrm{low}$, which suggested cis-diaxial orientation for these three pairs of protons in 19-low. In conjunction with the large coupling constant $(\mathrm{J}>=$ $10 \mathrm{~Hz}$ ) observed for $10-\mathrm{H} / 10 \mathrm{a}-\mathrm{H}$ and the absence of any NOE between $10-\mathrm{H}$ and 10a- $\mathrm{H}$ this suggested trans-diaxial configuration for $10-\mathrm{H}$ and $10 \mathrm{a}-\mathrm{H}$. For $19-$ high, the NOE's between $7-\mathrm{H}_{\mathrm{a}}$ and $9-\mathrm{H}_{\mathrm{a}}, 9-\mathrm{H}_{\mathrm{a}}$ and $10 \mathrm{a}-\mathrm{H}$, as well as $10 \mathrm{a}-\mathrm{H}$ and 7- $\mathrm{H}_{\mathrm{a}}$ were also observed, again indicating axial orientation of 10a-H. In 19-high, the $10-\mathrm{H} / 10 \mathrm{a}-\mathrm{H}$ coupling constant is small and a strong NOE was observed between $10-\mathrm{H}$ and $10 \mathrm{a}-\mathrm{H}$, indicating cis-gauche orientation for these protons.

\section{Spectral data for tetracyclic cycloadduct 20.}

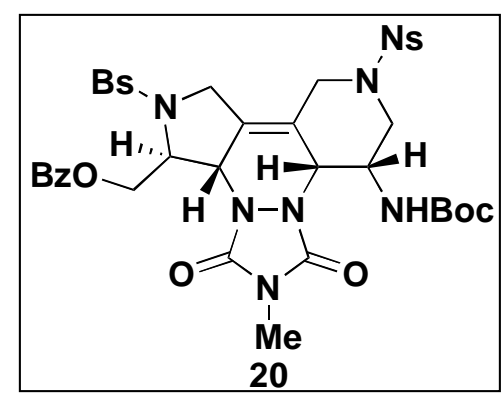

Tetracyclic Cycloadduct 20. Isolated as an amorphous off-white solid (35 mg, 93\% yield); IR (thin film/NaCl) 3430 (w), 2979 (w), 1775 (w), 1713 (s), 1544 (m), 
$1493(\mathrm{~m}), 1365(\mathrm{~m}), 1269(\mathrm{~m}), 1157(\mathrm{~s}), 1069(\mathrm{w}), 910(\mathrm{w}), 758(\mathrm{~m}), 730(\mathrm{~m}), 712$

(w) $\mathrm{cm}^{-1}$; $1 \mathrm{H}$ NMR $(500 \mathrm{MHz}, \mathrm{CDCl} 3) \delta \mathrm{ppm} 7.99$ (t, J=8.3 Hz, $\left.8 \mathrm{H}\right), 7.83$ (d, $J=8.3 \mathrm{~Hz}, 5 \mathrm{H}), 7.72-7.80(\mathrm{~m}, 6 \mathrm{H}), 7.66(\mathrm{t}, J=8.8 \mathrm{~Hz}, 3 \mathrm{H}), 7.58(\mathrm{t}, \mathrm{J}=7.3 \mathrm{~Hz}, 1$ H), 7.44 (t, J=7.6 Hz, 2 H), 5.28 (d, J=9.3 Hz, 1 H), 4.94 - 5.08 (m, 2 H), 4.85 (br. s., $1 \mathrm{H}), 4.66(\mathrm{~d}, J=8.8 \mathrm{~Hz}, 1 \mathrm{H}), 4.60$ (d, J=13.7 Hz, $1 \mathrm{H}$ ), 4.50 (br. s., $1 \mathrm{H}), 4.37$ (br. s., $1 \mathrm{H}$ ), 4.33 (d, J=14.6 Hz, 1 H), 3.88 (d, J=12.7 Hz, 1 H), 3.71 (d, J=14.6 Hz, $1 \mathrm{H}), 3.41$ (d, J=13.7 Hz, $1 \mathrm{H}), 3.13(\mathrm{~d}, \mathrm{~J}=12.7 \mathrm{~Hz}, 1 \mathrm{H}), 3.09(\mathrm{~s}, 3 \mathrm{H}), 1.43$ (s, $9 \mathrm{H}) ;{ }^{13} \mathrm{C}$ NMR $(100 \mathrm{MHz}, \mathrm{CDCl} 3) \delta 166.1,156,155.7,151.8,148.6,136.9$, 134.6, 133.6, 133.1, 132.2, 131.9, 131.6, 131, 130.1, 129.9, 129.8, 129.1, 128.8, 124.6, 119.8, 80.7, 65.9, 61.5, 58.2, 54, 49.8, 48.2, 48, 47.4, 28.6, 25.3; HRMS (ES+) $m / z$ 933.1547 [calc'd for $\mathrm{C}_{31} \mathrm{H}_{34} \mathrm{BrN}_{6} \mathrm{O}_{8} \mathrm{~S}\left(\mathrm{M}+\mathrm{NH}_{4}\right)$ 933.1547].

\section{Spectral data for Tetracyclic Cycloadduct 21.}

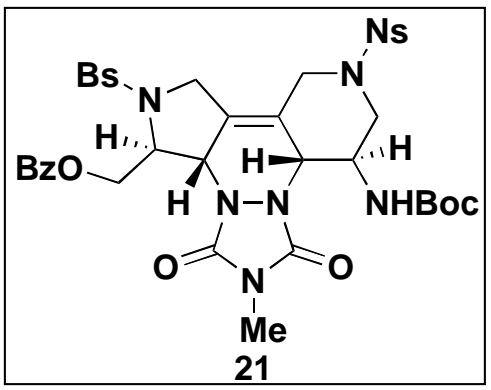

Tetracyclic Cycloadduct 21. Isolated as an amorphous off-white solid (48.5 mg, 90\% yield); IR (thin film/ $\mathrm{NaCl}$ ) 3430 (w), 2979 (w), 1775 (w), 1713 (s), 1547 (m), $1470(\mathrm{~m}), 1367(\mathrm{~m}), 1269(\mathrm{~m}), 1164(\mathrm{~s}), 1069(\mathrm{w}), 759(\mathrm{~m}), 747(\mathrm{~m}), 713(\mathrm{w}) \mathrm{cm}^{-}$ 1; $1 \mathrm{H}$ NMR (500 MHz, CDCl3) $\delta$ ppm 8.01 (d, J=7.3 Hz, 1 H), 7.94 (d, J=6.8 Hz, 2 H), $7.71-7.83(\mathrm{~m}, 6 \mathrm{H}), 7.66(\mathrm{~d}, \mathrm{~J}=7.3 \mathrm{~Hz}, 1 \mathrm{H}), 7.57$ (t, J=6.8 Hz, $1 \mathrm{H}), 7.43$ (t, J=7.3 Hz, 2 H), 5.01 (d, J=11.2 Hz, 1 H), 4.92 (d, J=7.3 Hz, 1 H), 4.85 (d, J=10.7 
Hz, 1 H), 4.72 (br. s., 1 H), 4.57 - $4.67(m, 1 H), 4.40(d, J=14.2 \mathrm{~Hz}, 1 \mathrm{H}), 4.32(\mathrm{~d}$, J=7.8 Hz, 1 H), 4.19 (br. s., 1 H), 4.05 (d, J=14.6 Hz, 1 H), 3.87 (d, J=10.2 Hz, 1 H), 3.30 (d, J=12.7 Hz, 1 H), 3.15 (br. s., 1 H), 3.00 (s, 3 H), 2.80 (t, J=11.2 Hz, 1 H), $1.42(\mathrm{~s}, 9 \mathrm{H}) ;{ }^{13} \mathrm{C} \mathrm{NMR}(100 \mathrm{MHz}, \mathrm{CDCl} 3) \delta 166.1,157.9,154.6,151.8$, $148.7,136.8,134.7,133.7,133.5,132.3,132.1,131.6,130.4,130.3,129.8$, 129.2, 128.8, 124.6, 121.4, 80.4, 66.7, 61.9, 60.7, 55.9, 52.6, 49.2, 49.1, 47.3, 28.5, 25.6; HRMS (ES+) $\mathrm{m} / \mathrm{z} 933.1509$ [calc'd for $\mathrm{C}_{31} \mathrm{H}_{34} \mathrm{BrN}_{6} \mathrm{O}_{8} \mathrm{~S}\left(\mathrm{M}+\mathrm{NH}_{4}\right)$ 933.1547].

The relative configurations of $\mathbf{2 0}$ and $\mathbf{2 1}$ at the carbon bearing the $\mathrm{CH}_{2}-$ OBz substituent were assigned based on strong NOE's observed for the bridgehead protons and the $\mathrm{CH}_{2}-\mathrm{OBz}$ protons in both 20 and 21 . The relative configurations at the BocNH-bearing carbon were assigned based on observation of a strong NOE and a small coupling constant for the $\mathrm{CH}-\mathrm{NHBoc}$ proton and the adjacent bridgehead proton in 20 , whereas in 21 the $\mathrm{CH}-\mathrm{NHBoc}$ proton and the adjacent bridgehead proton exhibit strong coupling $(\mathrm{J}=10 \mathrm{~Hz})$ and there is no NOE between these protons. This indicated trans-diaxial orientation of these two protons in $\mathbf{2 1}$ and cis-gauche orientation in $\mathbf{2 0 .}$

\section{Spectral data for pentacyclic cycloadduct 22.}

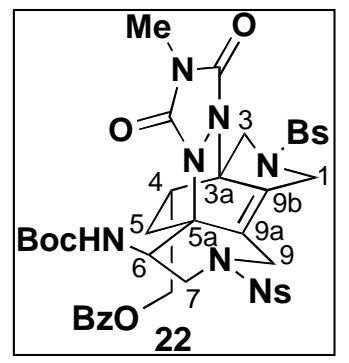


Pentacyclic cycloadduct 22. Isolated as an amorphous white solid (11 mg, 46\% yield); IR (thin film/NaCl) 3367 (w), 2976 (w), 1697 (s), 1574 (w), 1544 (m), 1502 (w), $1458(m), 1390(w), 1364(m), 1315(w), 1269(m), 1168(s), 1113(w), 1069$ (w), $1025(w), 1008(w), 957(w), 739(m), 712(m) c^{-1}$; 1H NMR $(500 \mathrm{MHz}$, CDCl3) $\delta$ ppm 8.05 (d, J=7.8 Hz, 1 H), 7.95 (d, J=7.8 Hz, 2 H), 7.57 - 7.78 (m, 8 H), 7.49 (t, J=7.6 Hz, 2 H), $6.63(\mathrm{~d}, J=8.8 \mathrm{~Hz}, 1 \mathrm{H}), 4.52$ - $4.63(\mathrm{~m}, 1 \mathrm{H}), 4.49$ (d, $J=11.7 \mathrm{~Hz}, 1 \mathrm{H}), 4.24-4.34(\mathrm{~m}, 1 \mathrm{H}), 4.13-4.22(\mathrm{~m}, 2 \mathrm{H}), 4.10$ (d, J=12.2 Hz, 1 H), 3.95 - $4.06(\mathrm{~m}, 3 \mathrm{H}), 3.66(\mathrm{t}, \mathrm{J}=11.5 \mathrm{~Hz}, 1 \mathrm{H}), 3.50$ - $3.61(\mathrm{~m}, 1 \mathrm{H}), 2.99(\mathrm{~s}, 3$ H), 2.75 (br. s., $1 \mathrm{H}$ ), 2.51 (dd, J=13.2, $9.8 \mathrm{~Hz}, 1 \mathrm{H}$ ), 1.42 (bs, $10 \mathrm{H}$ ); ${ }^{13} \mathrm{C}$ NMR (125 MHz, CDCl3) $\delta 166,155.8,152.4,151.9,148.1,135.5,134.2,133.9,133.8$, 132.8, 132.2, 131.8, 131.7, 129.9, 129.7, 129.6, 129.5, 129.2, 128.8, 124.6, 80.6, $66.7,63.8,62.7,50.2,48.3,48,44.8,41.2,37.9,33.7,28.2,25.6$; HRMS (ES+) $m / z 959.1667$ [calc'd for $\mathrm{C}_{39} \mathrm{H}_{40} \mathrm{BrN}_{7} \mathrm{O}_{12} \mathrm{~S}_{2}\left(\mathrm{M}+\mathrm{NH}_{4}\right)$ 959.1703].

Stereochemical assignment of $\mathbf{2 2}$ was based on the observation of a strong NOE between $9-\mathrm{H}_{\mathrm{a}}$ and $6-\mathrm{H}$, indicating 1,4-diaxial configuration of these two protons, which implies boat conformation of the corresponding 6-membered ring. In conjunction with the observation of strong NOE's between $6-\mathrm{H}$ and $5-\mathrm{H}_{\mathrm{a}}$, $5-\mathrm{H}_{\mathrm{a}}$ and $4-\mathrm{CH}_{\mathrm{a}} \mathrm{H}_{\mathrm{b}}-\mathrm{OBz}$, as well as $4-\mathrm{H}$ and $5-\mathrm{H}_{\mathrm{b}}$ the axial orientation of $6-\mathrm{H}$ requires that the benzoyloxymethyl substituent in position 4 is oriented trans to the Boc-amino substituent in position 6. Full NMR-spectroscopic assignments for the protons and carbons representing the primary tricyclic core of $\mathbf{2 2}$ are given in Table 6.

Table 6. NMR data for $22\left(600 \mathrm{MHz}\right.$ for ${ }^{1} \mathrm{H}, 151 \mathrm{MHz}$ for $\left.{ }^{13} \mathrm{C}, \mathrm{CDCl} 3\right)$. 


\begin{tabular}{|c|c|c|c|}
\hline Carbon & $\delta\left({ }^{13} \mathrm{C}\right) / \mathrm{ppm}$ & proton & $\delta\left({ }^{1} \mathrm{H}\right) / \mathrm{ppm}$ \\
\hline $\mathrm{C}-1$ & 48.18 & $1-\mathrm{H}_{\mathrm{a}}$ & 3.99 \\
\hline & & $1-\mathrm{H}_{\mathrm{b}}$ & 3.99 \\
\hline $\mathrm{C}-3$ & 50.42 & $3-\mathrm{H}_{\mathrm{a}}$ & 4.09 \\
\hline & & $3-\mathrm{H}_{\mathrm{b}}$ & 4.49 \\
\hline $\mathrm{C}-3 \mathrm{a}$ & 66.93 & & \\
\hline $\mathrm{C}-4$ & 38.14 & $4-\mathrm{H}$ & 2.75 \\
\hline $4-\mathrm{CH}_{2}-\mathrm{OBz}$ & 64.04 & $\mathrm{CH}_{\mathrm{a}} \mathrm{H}_{\mathrm{b}}-\mathrm{OBz}$ & 4.01 \\
\hline & & $\mathrm{CH}_{\mathrm{a}} \mathrm{H}_{\mathrm{b}}-\mathrm{OBz}$ & 4.17 \\
\hline $\mathrm{C}-5$ & 33.90 & $5-\mathrm{H}_{\mathrm{a}}$ & 1.41 \\
\hline & & $5-\mathrm{H}_{\mathrm{b}}$ & 2.50 \\
\hline $\mathrm{C}-5 \mathrm{a}$ & 62.94 & & \\
\hline $\mathrm{C}-6$ & 48.51 & $6-\mathrm{H}$ & 4.56 \\
\hline & & $6-\mathrm{NH}$ & 6.62 \\
\hline $\mathrm{C}-7$ & 45.03 & $7-\mathrm{Ha}$ & 3.57 \\
\hline & & $7-\mathrm{Hb}$ & 3.65 \\
\hline $\mathrm{C}-9$ & 41.56 & $9-\mathrm{H}_{\mathrm{a}}$ & 4.28 \\
\hline $\mathrm{C}-9 \mathrm{a}$ & 129.62 & $9-\mathrm{H}_{\mathrm{b}}$ & 4.56 \\
\hline $\mathrm{C}-9 \mathrm{~b}$ & 135.48 & & \\
\hline
\end{tabular}


Spectra. Spectral Data for 4-tert-Butoxycarbonylamino-2-butyn-1-ol (2).

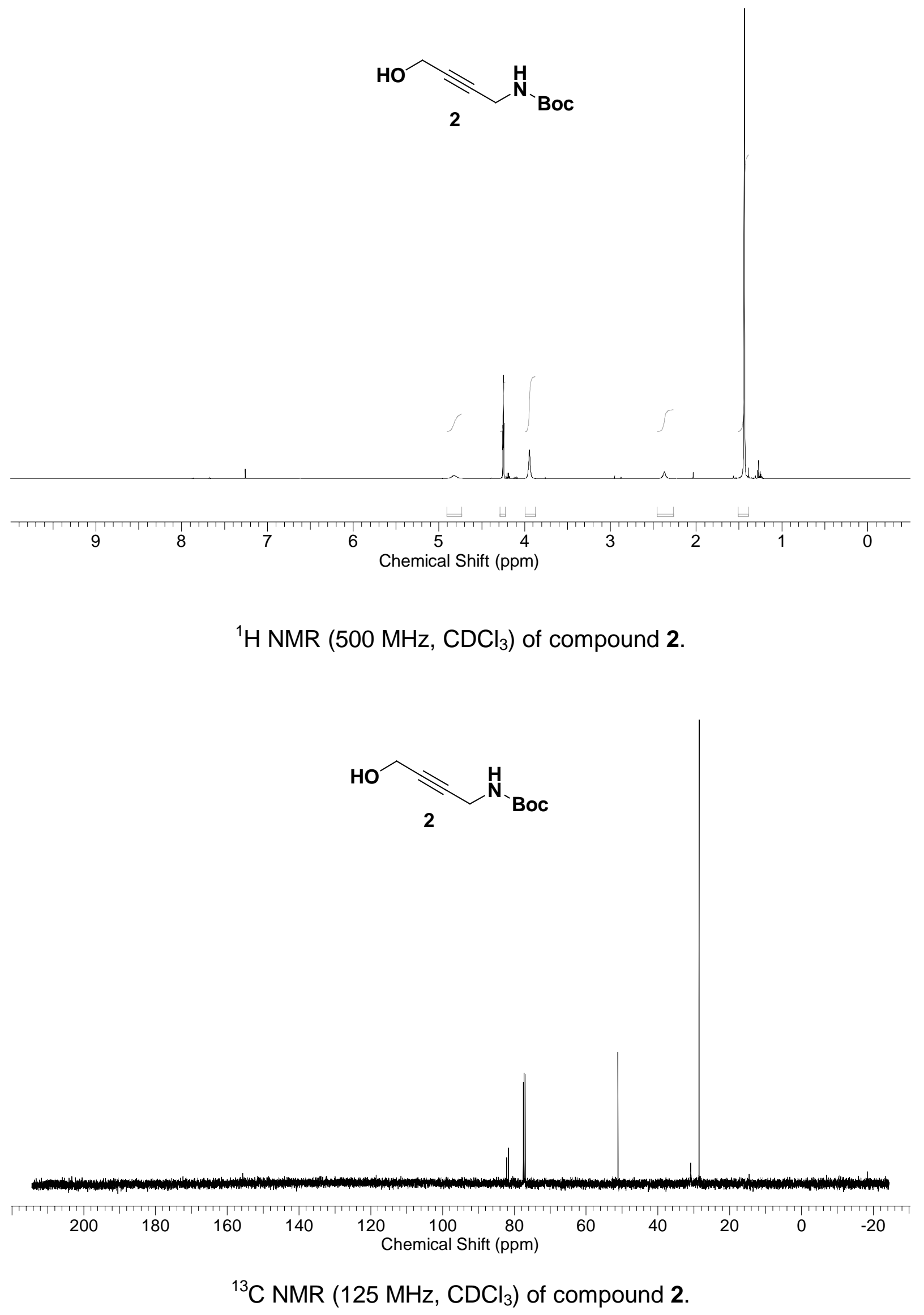


Spectral Data for 4-(4-Bromophenylsulfonamido)but-2-ynyl benzoate (4).

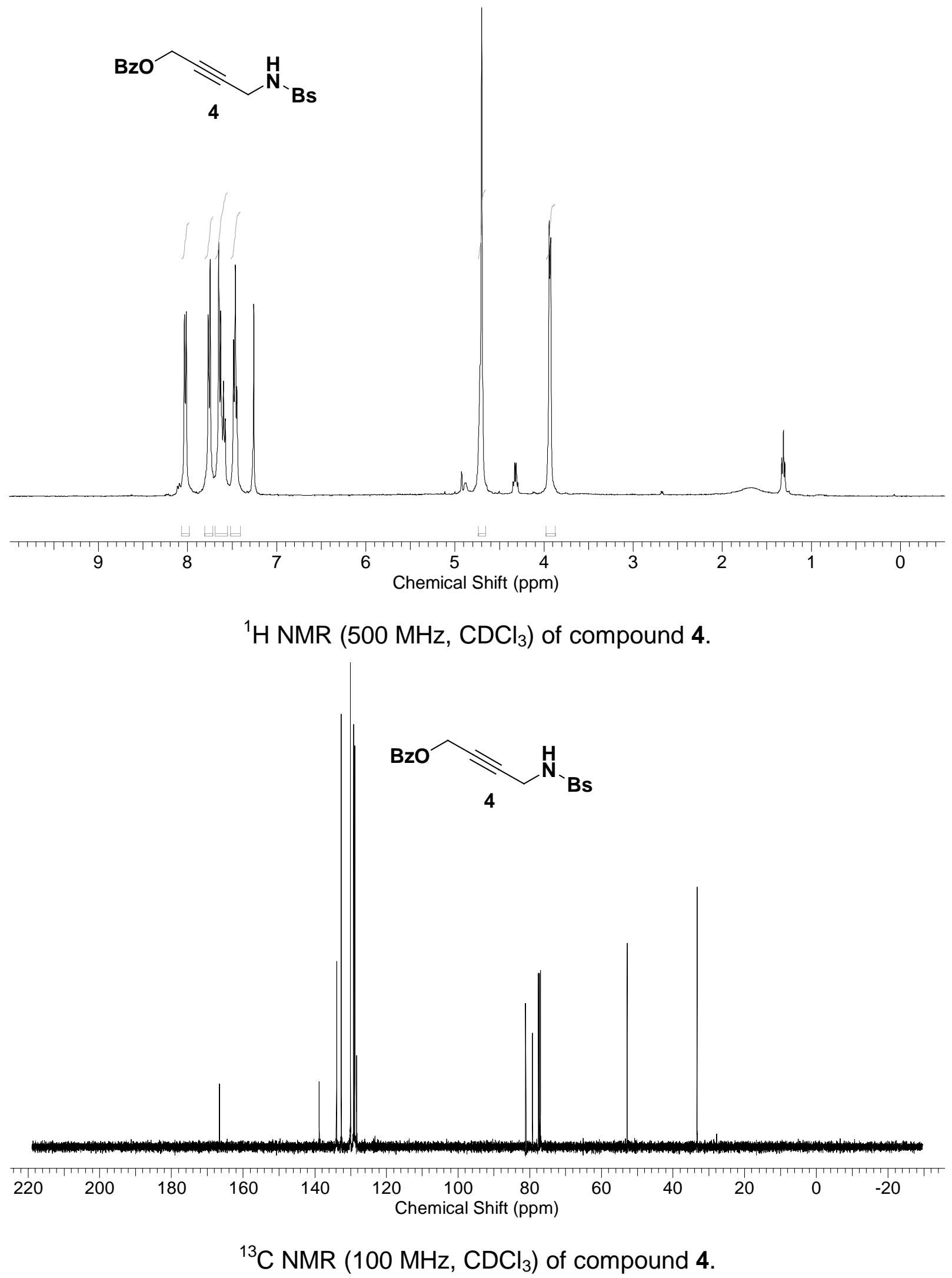


Spectral Data for (S)-2-(4-Bromophenylsulfonamido)but-3-enyl benzoate ((S)-3).
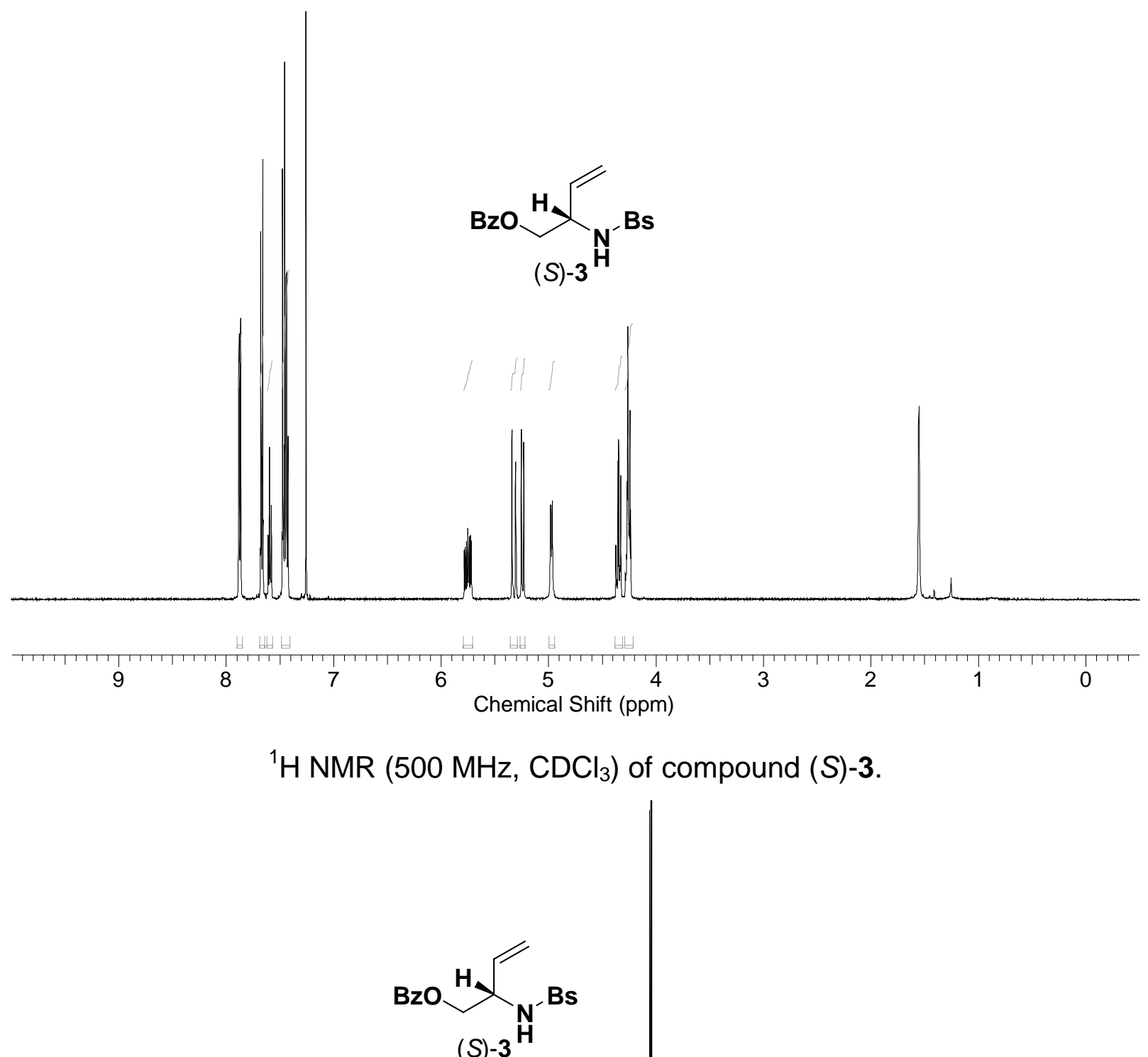

$(S)-3^{H}$

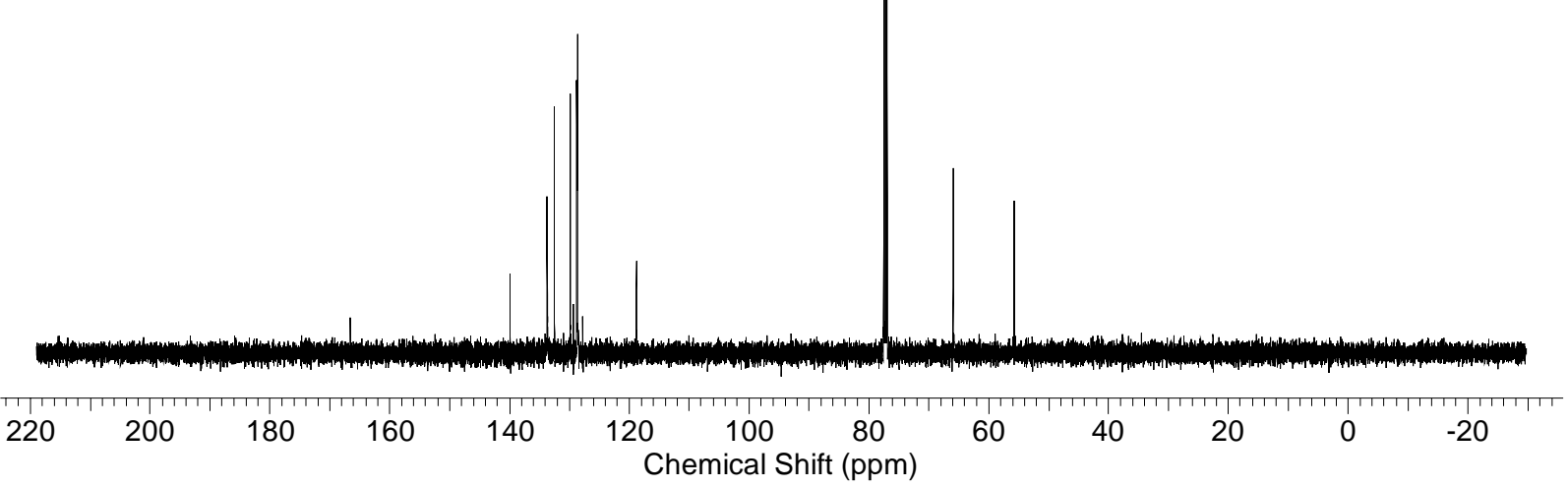

${ }^{13} \mathrm{C} \mathrm{NMR}\left(100 \mathrm{MHz}, \mathrm{CDCl}_{3}\right)$ of compound $(S)-3$. 
Spectral Data for $(S, R)$-N-Boc-ene-ene $((S, R)-5)$.

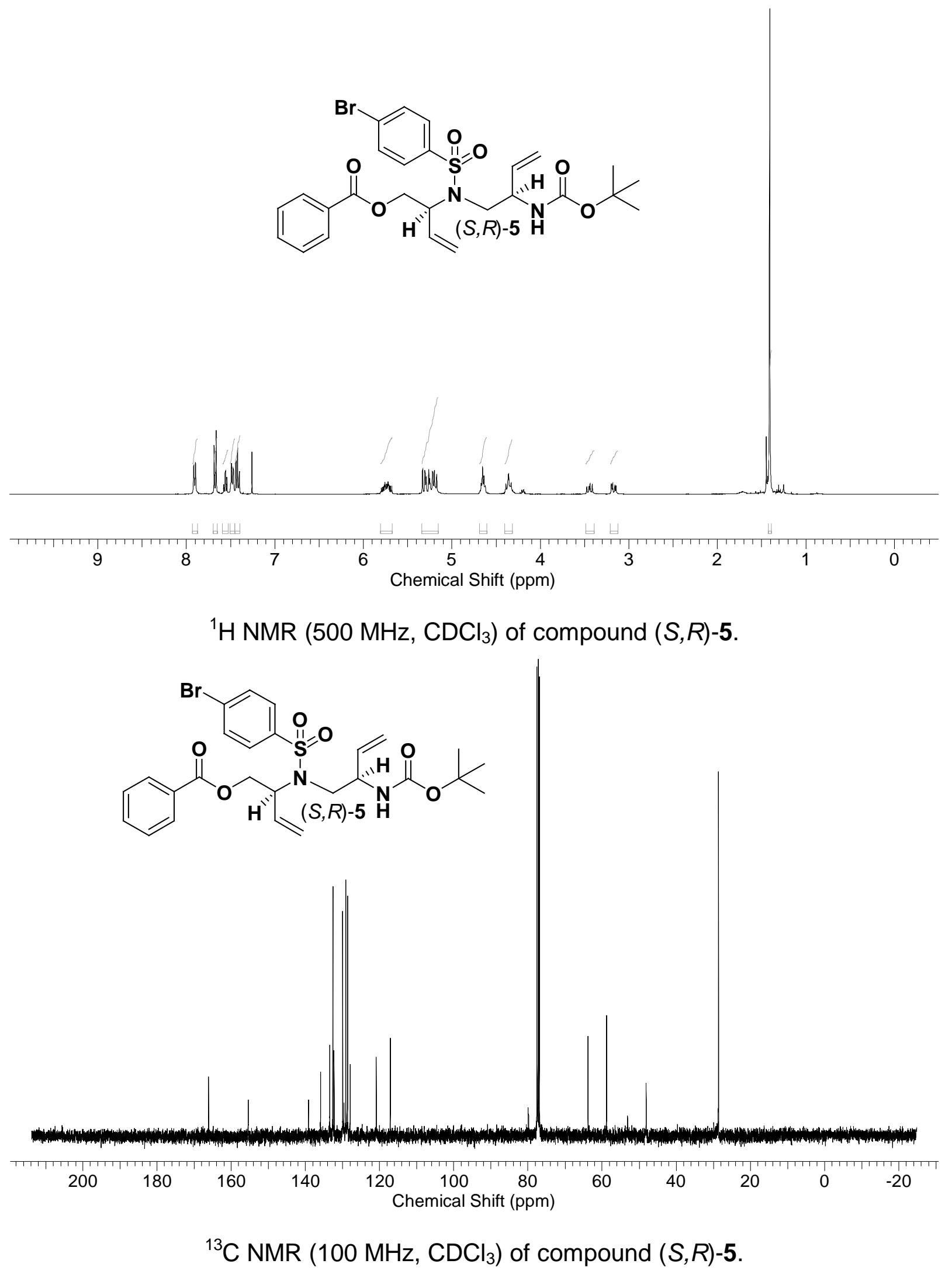

Spectral Data for $(R, R)-\mathrm{N}-\mathrm{Boc}-\mathrm{ene}-\mathrm{ene}((R, R)-5)$. 

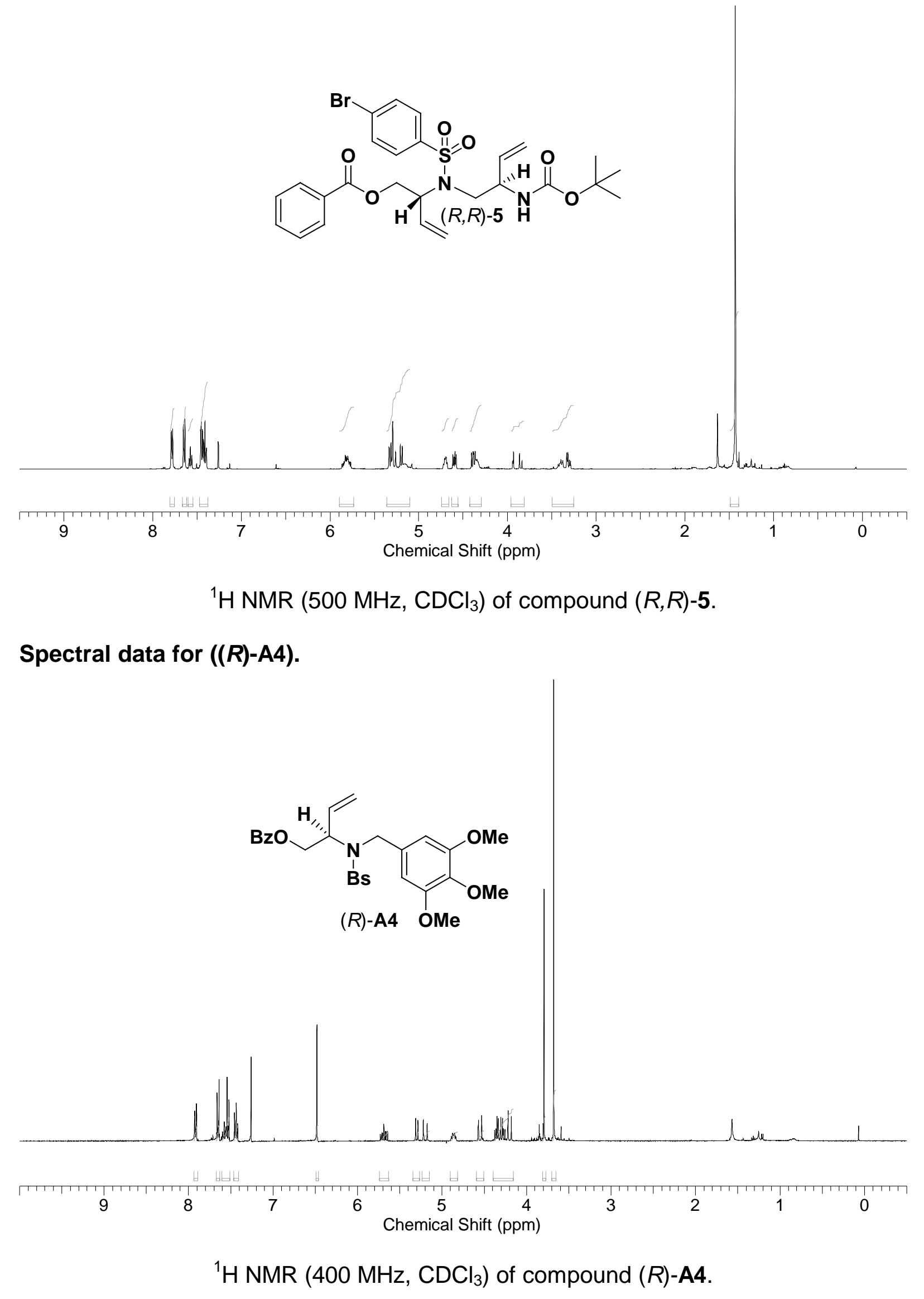


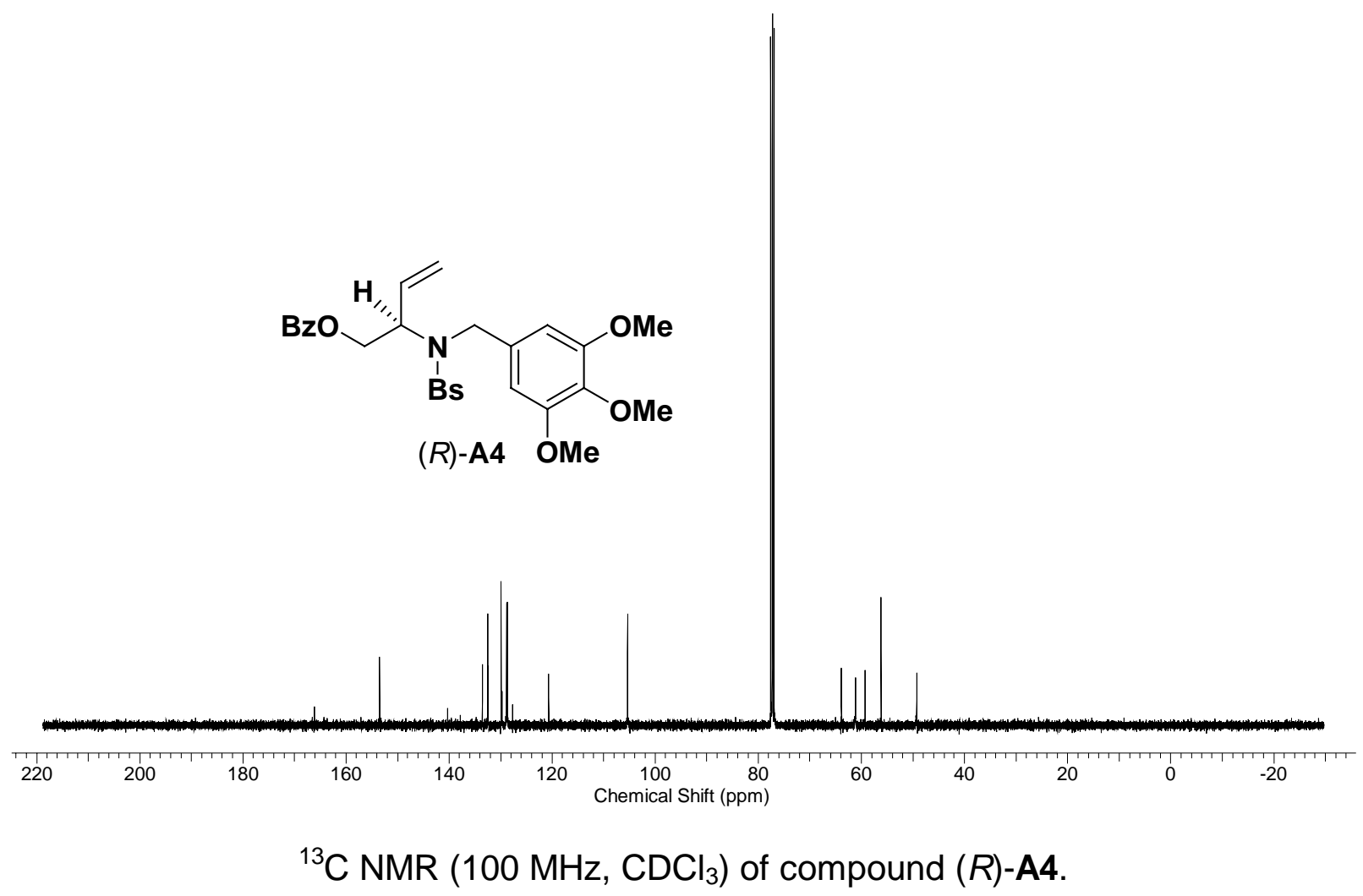

Spectral Data for (S)-N-Boc-ene-yne 6.

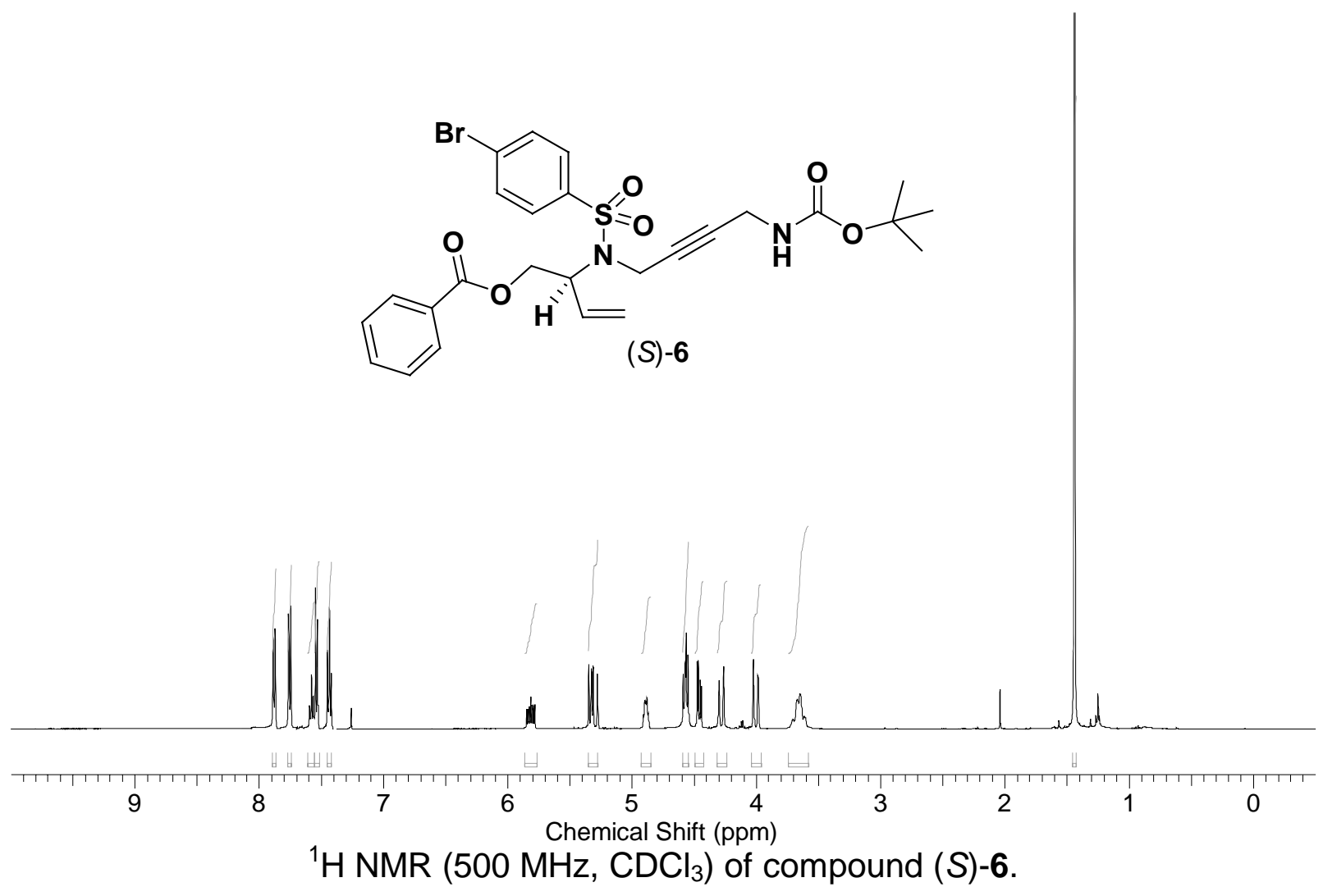




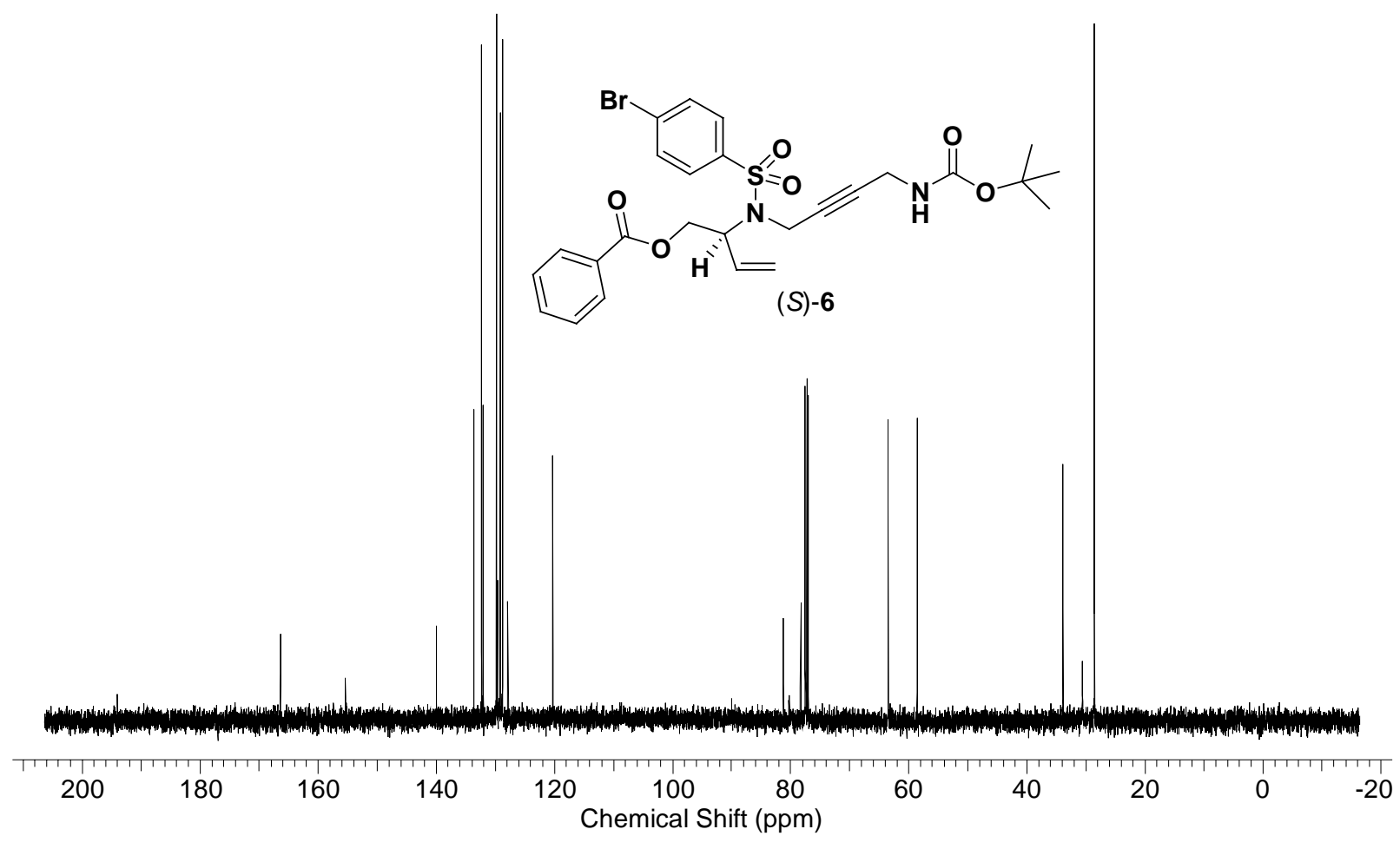

${ }^{13} \mathrm{C}$ NMR (125 MHz, $\mathrm{CDCl}_{3}$ ) of compound (S)-6.

Spectral Data for (rac)-N-Boc-yne-ene 7.

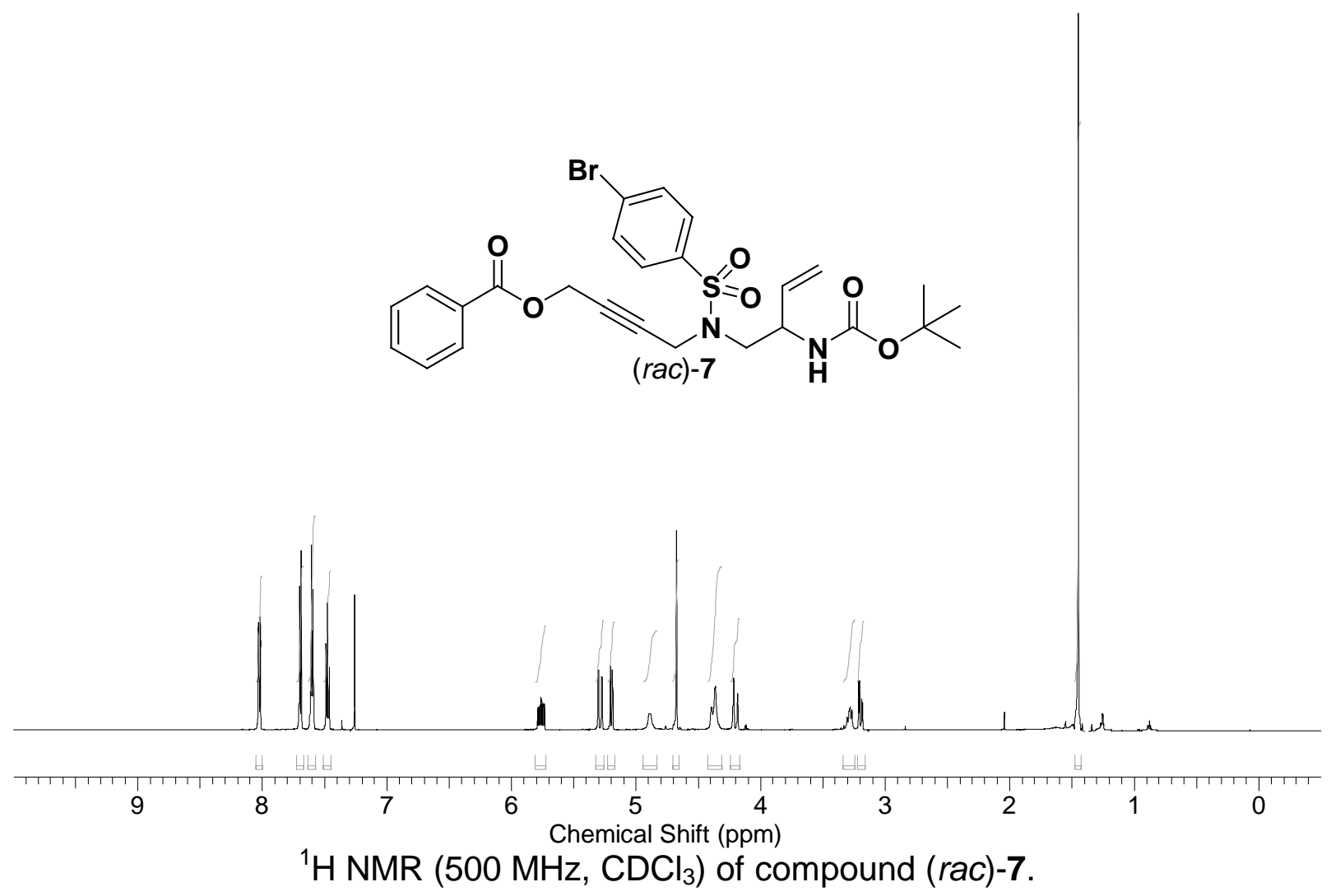




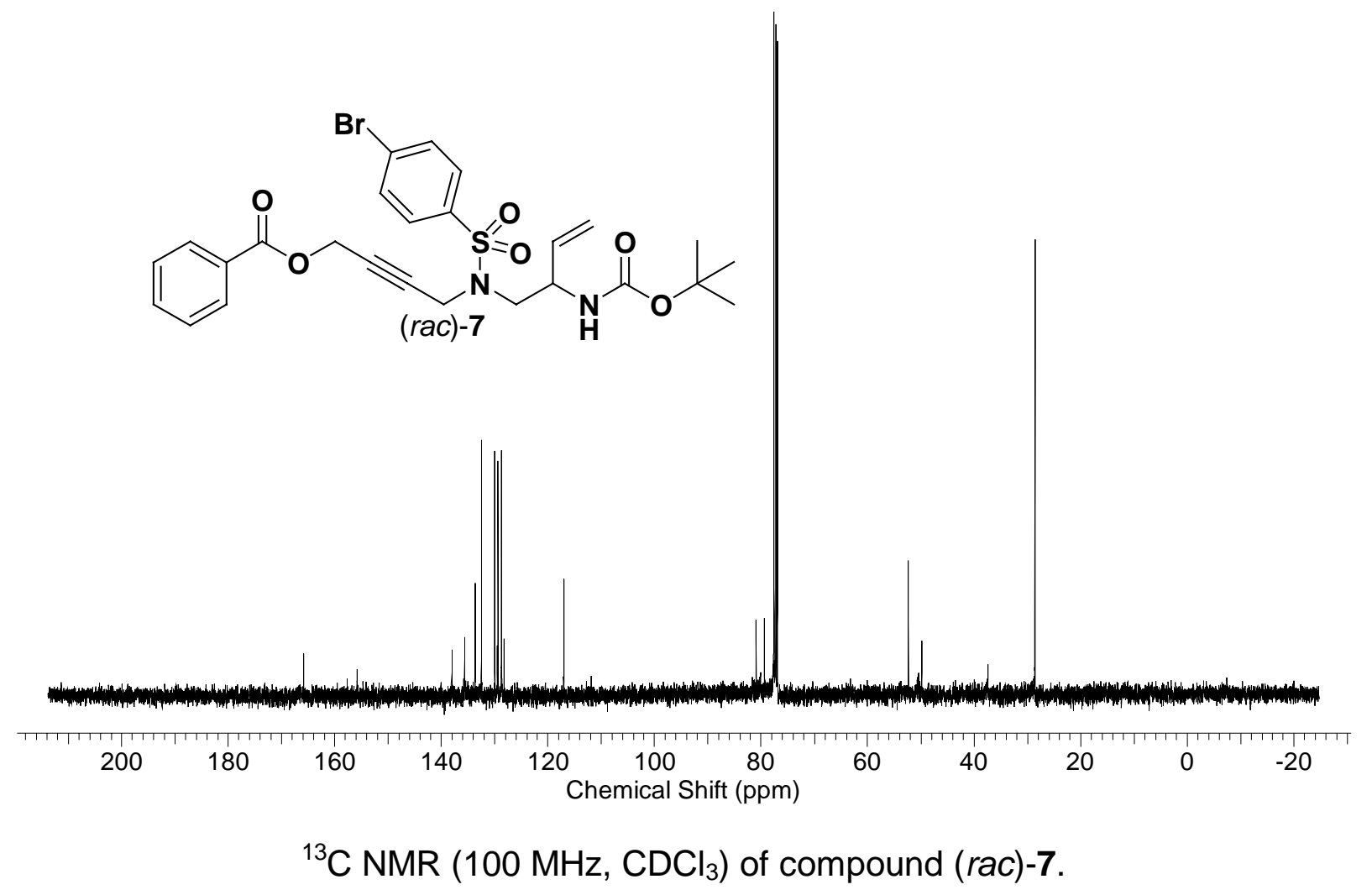

Spectral Data for N-Boc-yne-yne 8.

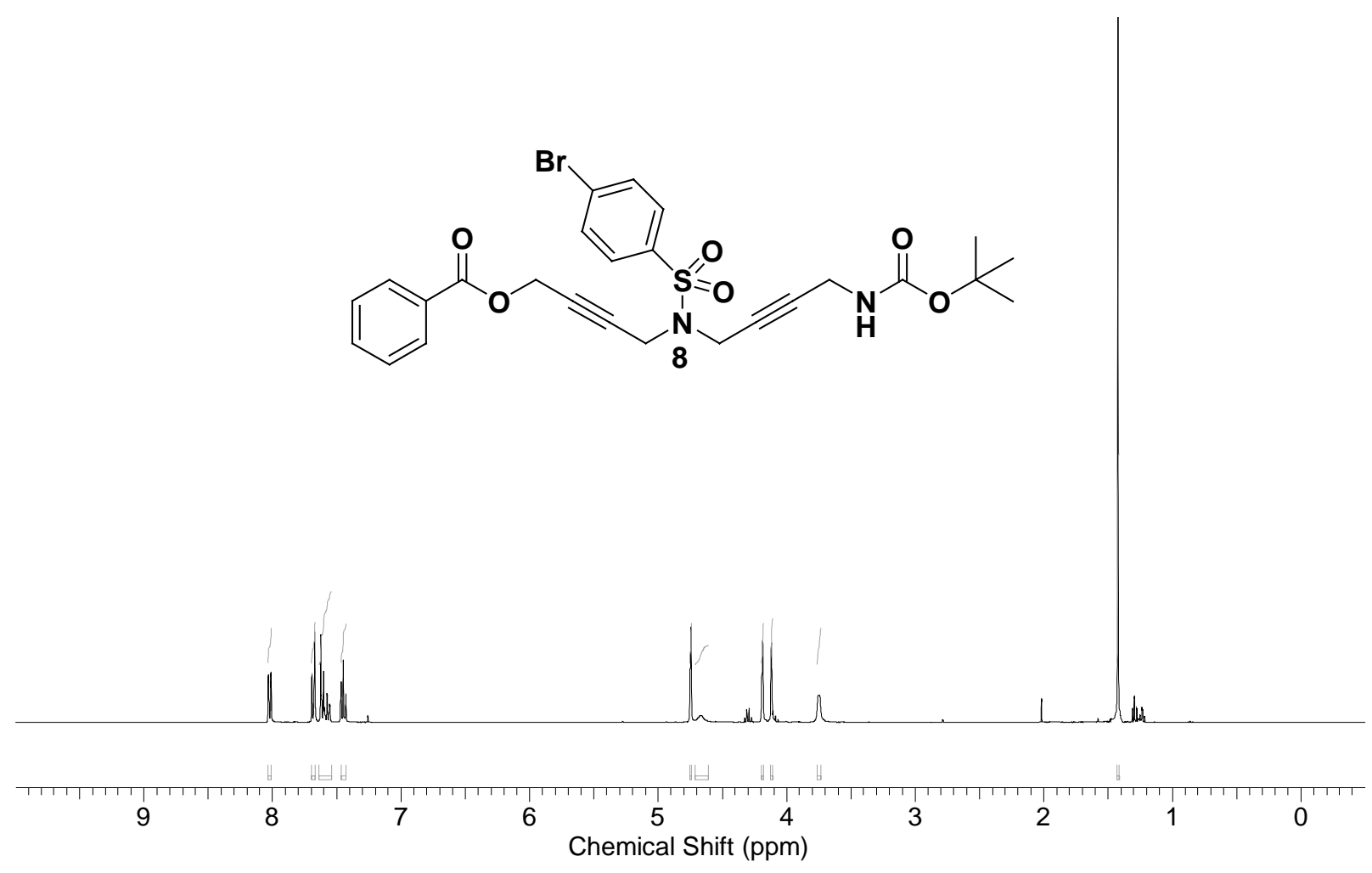

${ }^{1} \mathrm{H}$ NMR $\left(500 \mathrm{MHz}, \mathrm{CDCl}_{3}\right.$ ) of compound 8. 


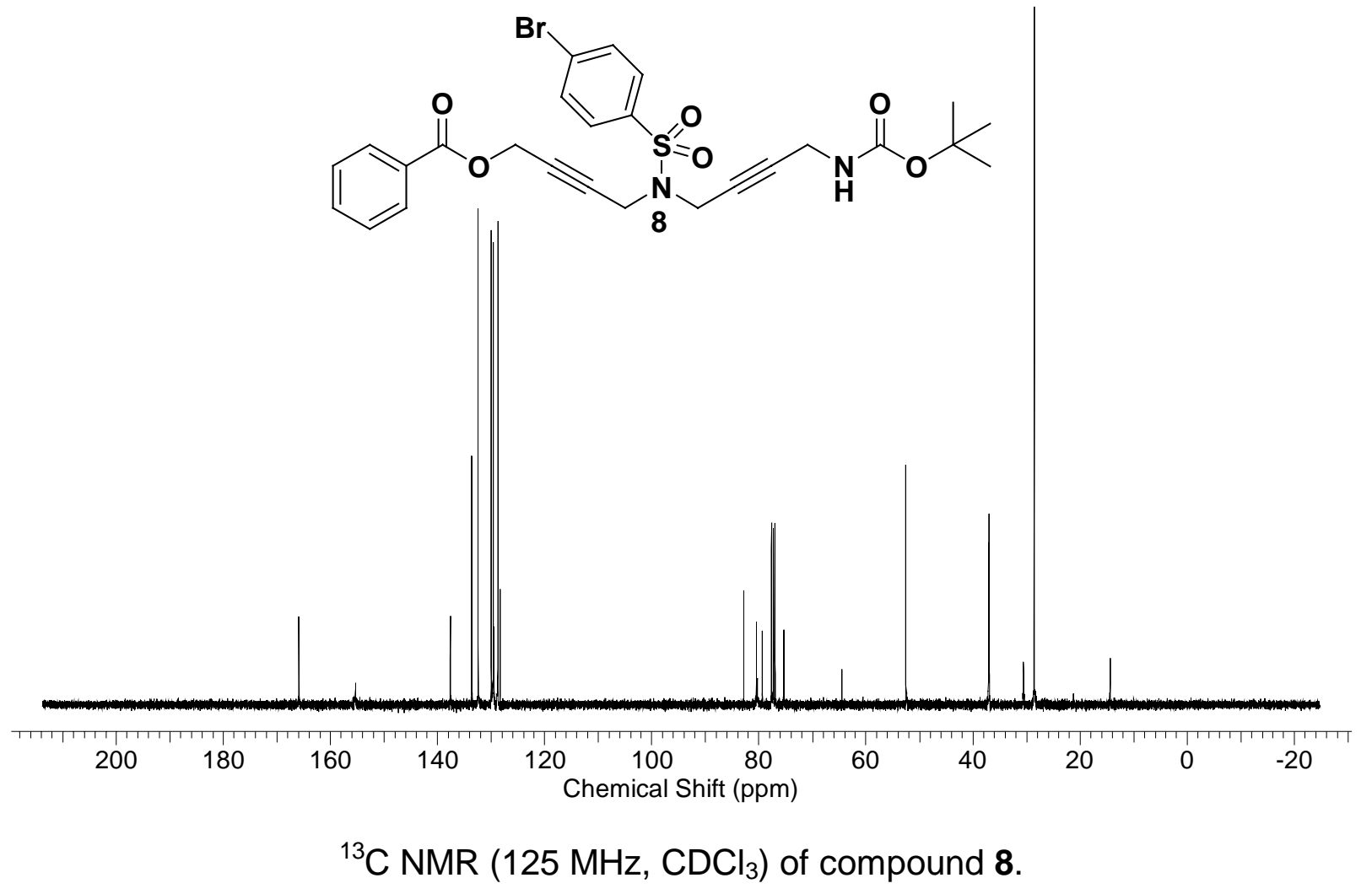

Spectral Data for Tetrahydropyridine (S,R)-9.

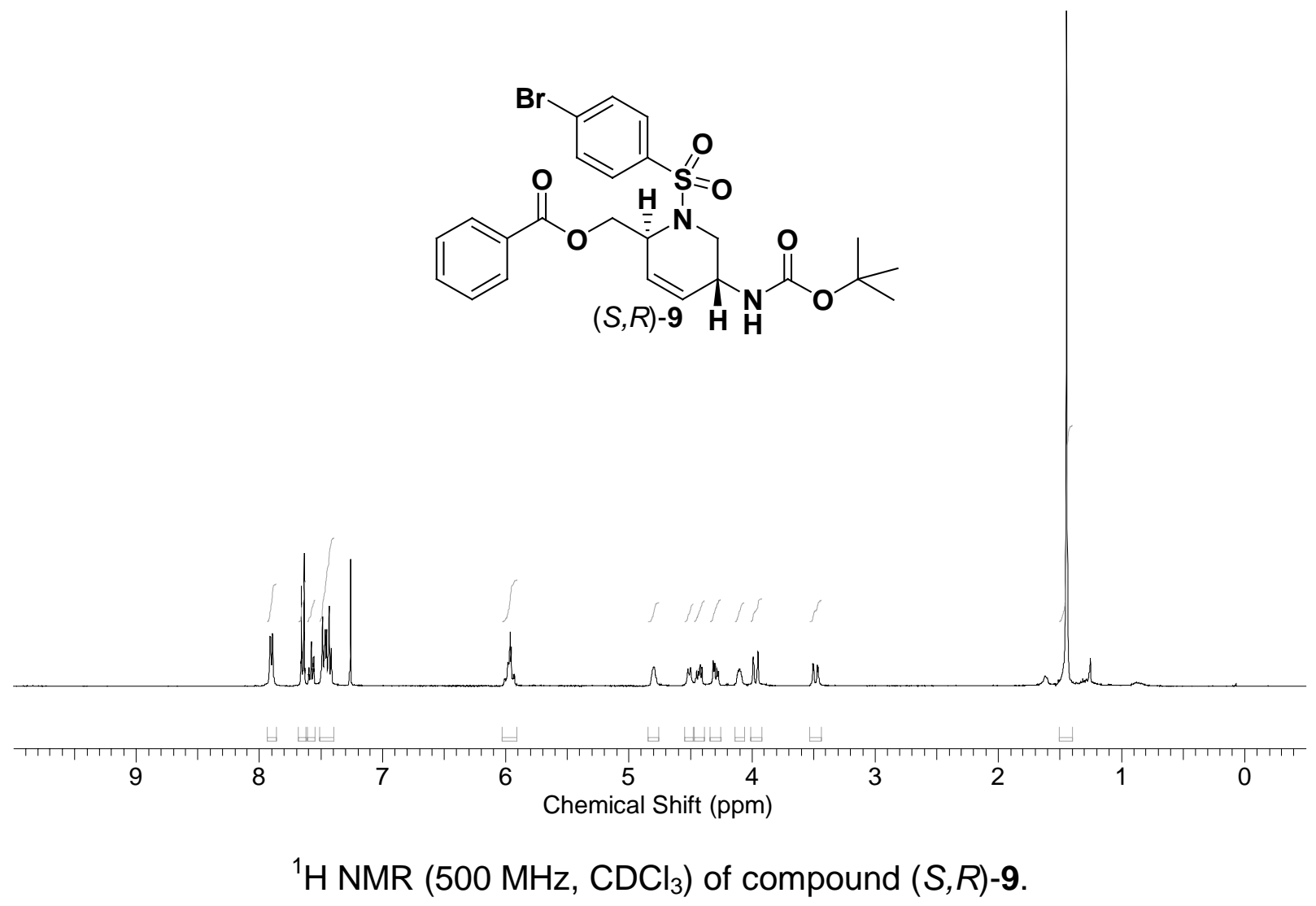



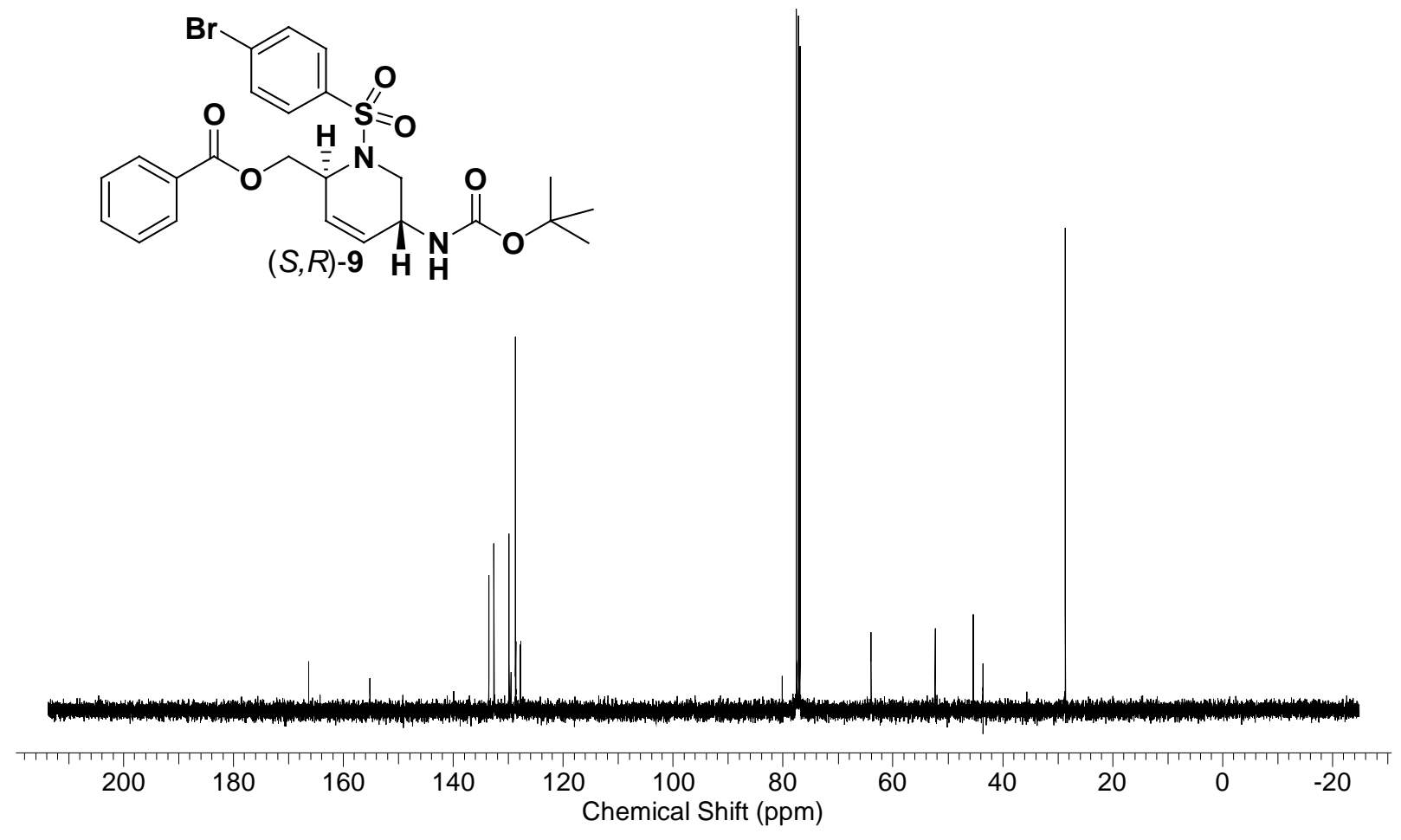

${ }^{13} \mathrm{C}$ NMR (125 MHz, $\mathrm{CDCl}_{3}$ ) of compound $(S, R)-9$.

Spectral Data for Tetrahydropyridine $(R, R)-9$.

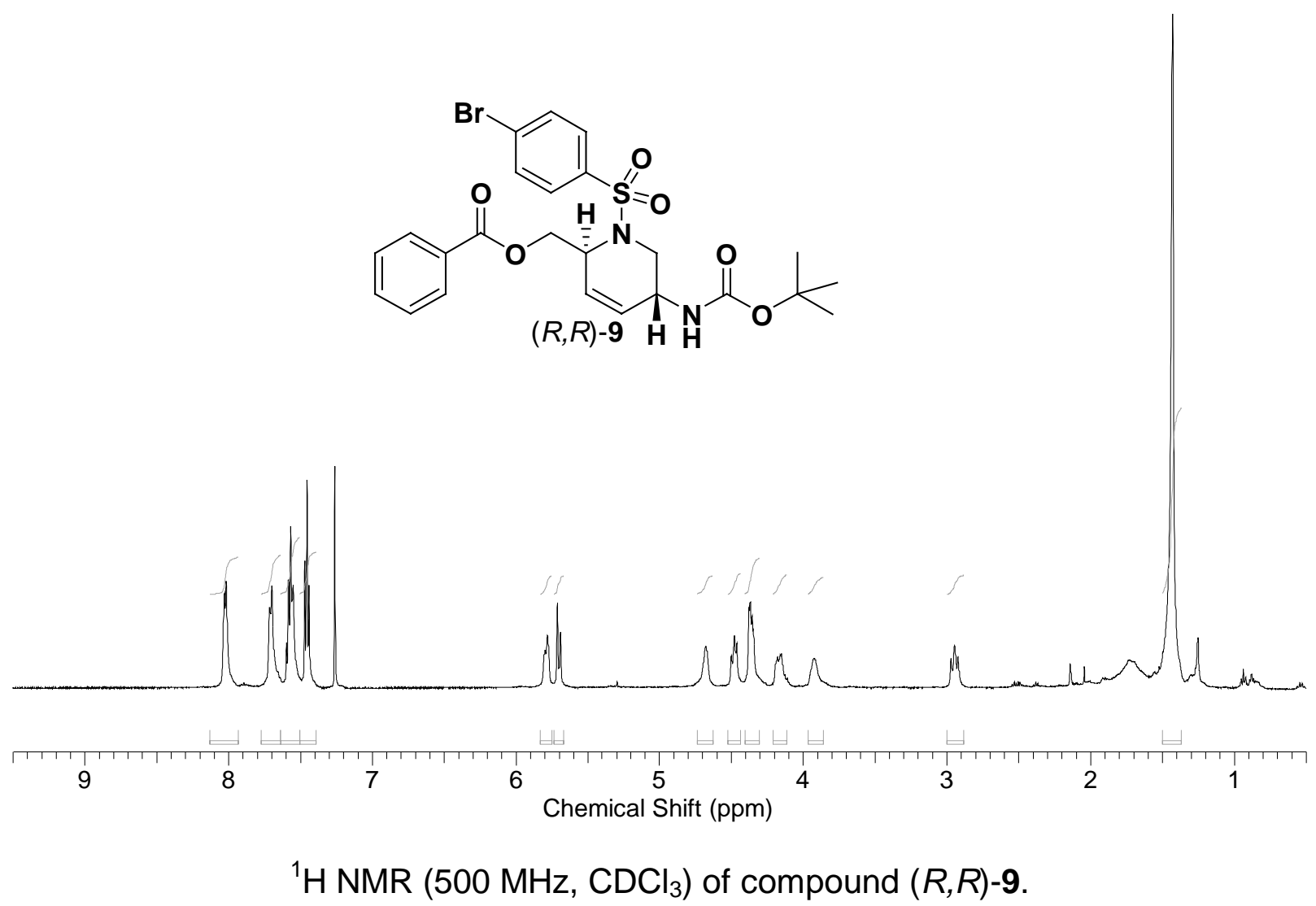




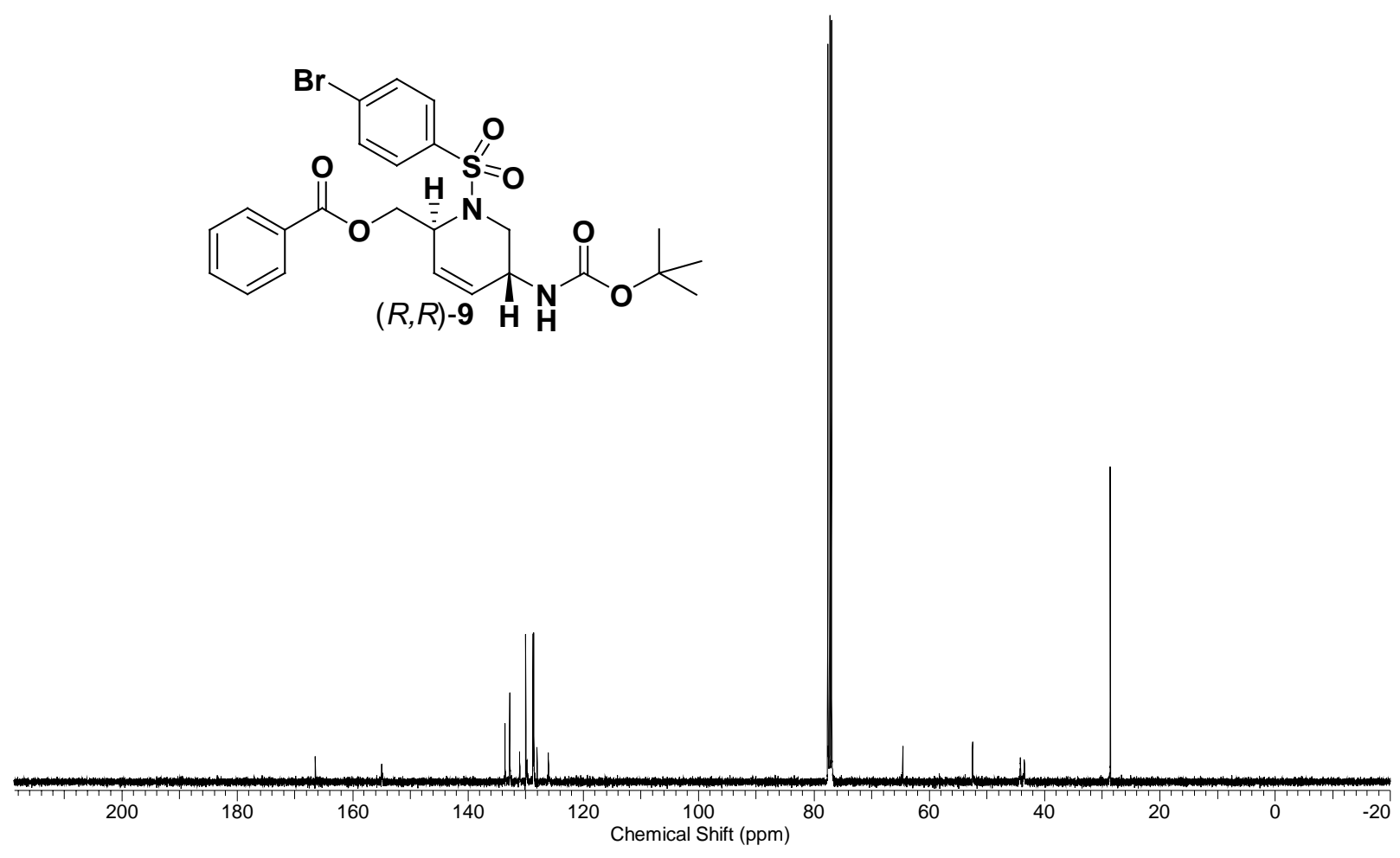

${ }^{13} \mathrm{C}$ NMR $\left(125 \mathrm{MHz}, \mathrm{CDCl}_{3}\right)$ of compound $(R, R)-\mathbf{9}$.

\section{Spectral Data for (rac)-Dihydropyrrole 10.}

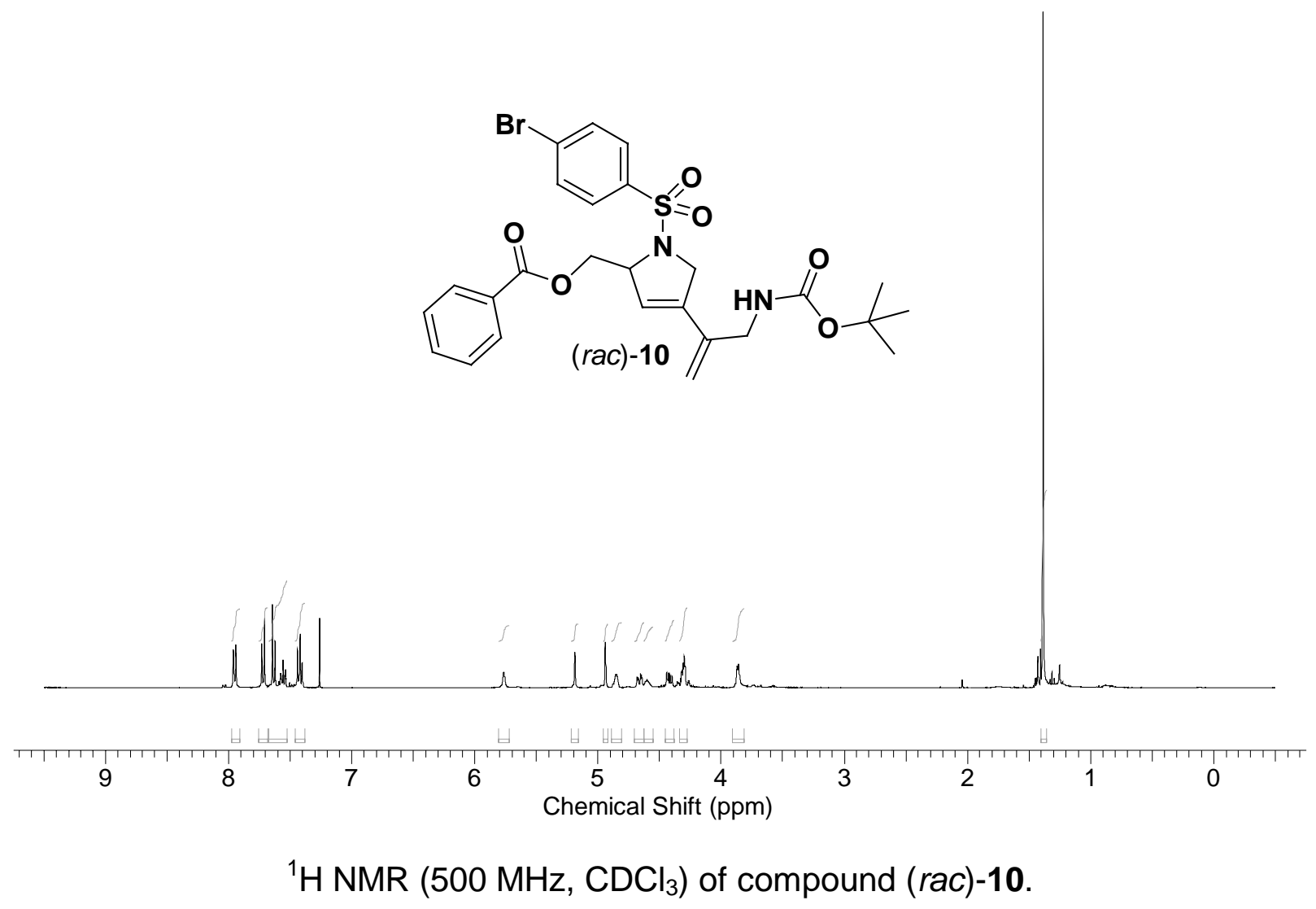




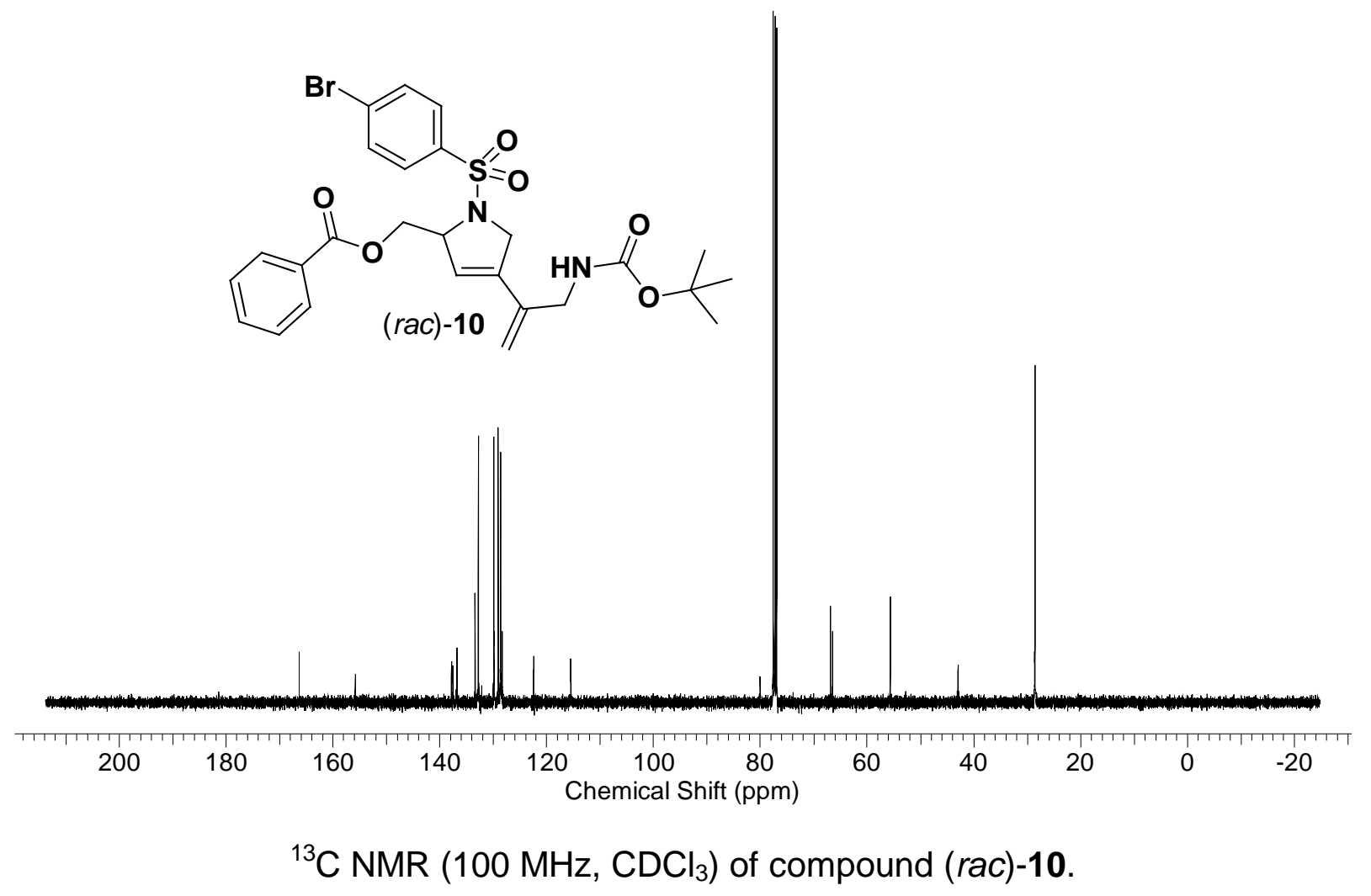

Spectral Data for (rac)-Tetrahydropyridine 11.

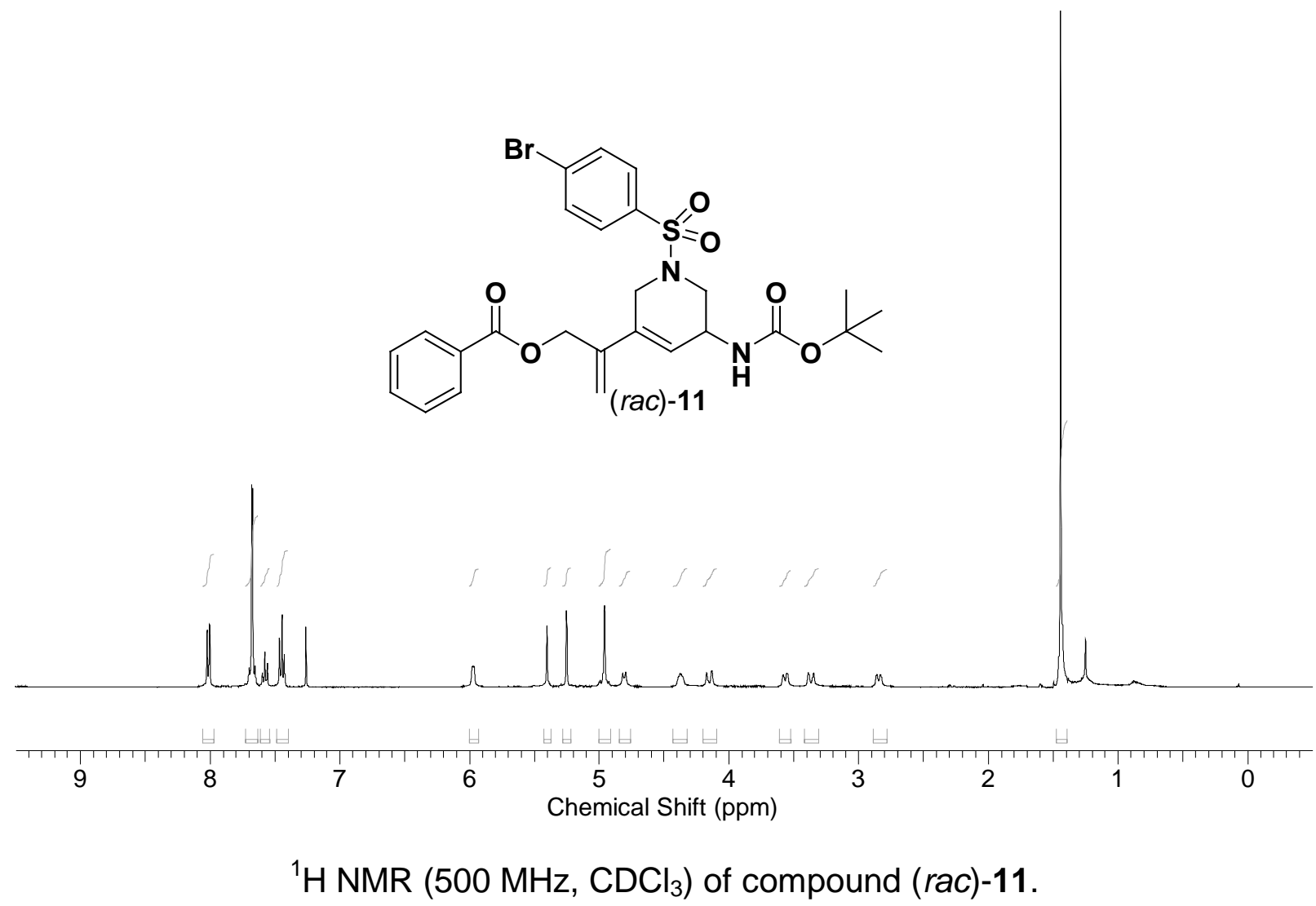




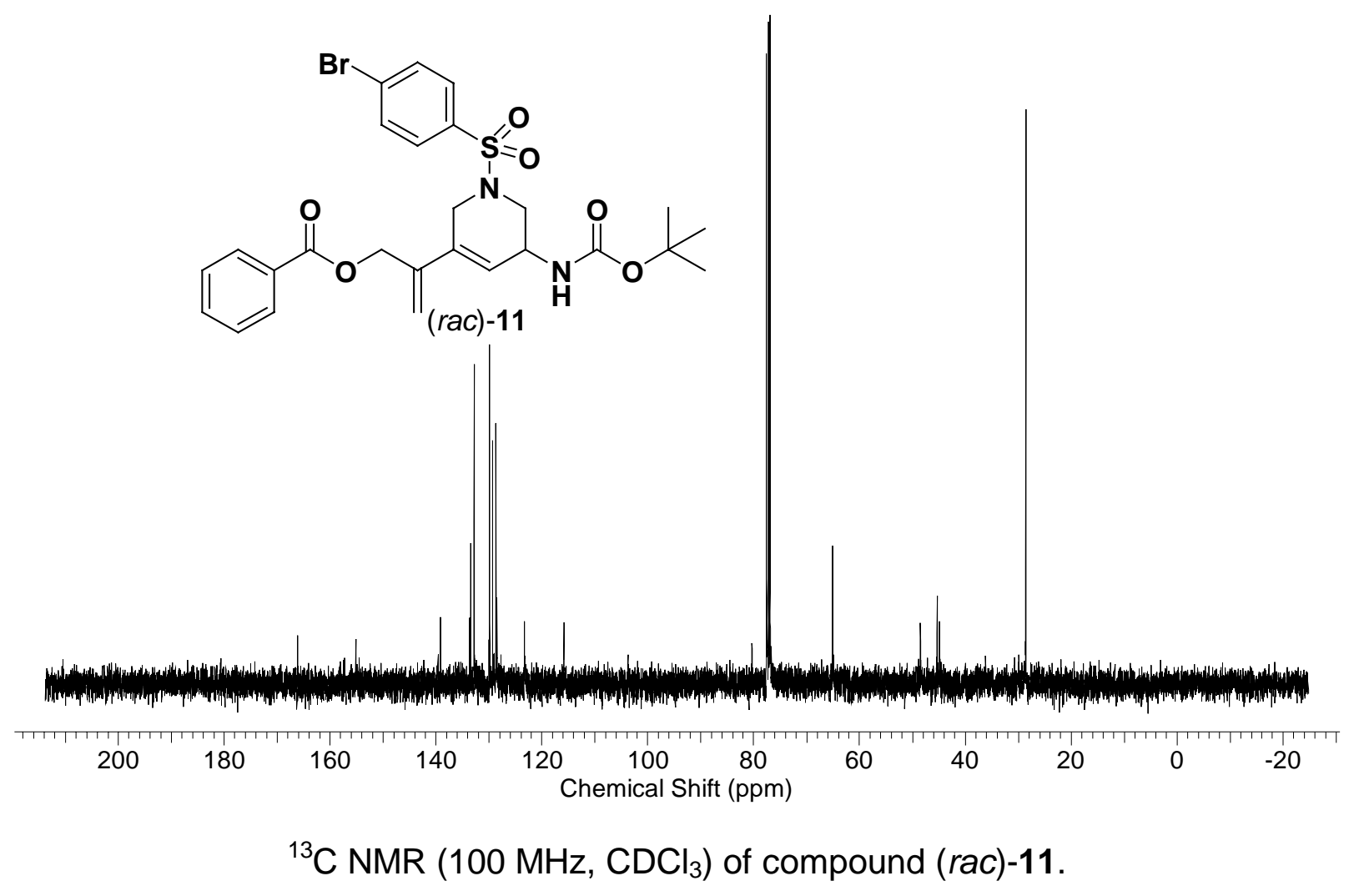

Spectral Data for N-Boc-ene-yne-ene (S,R)-12.

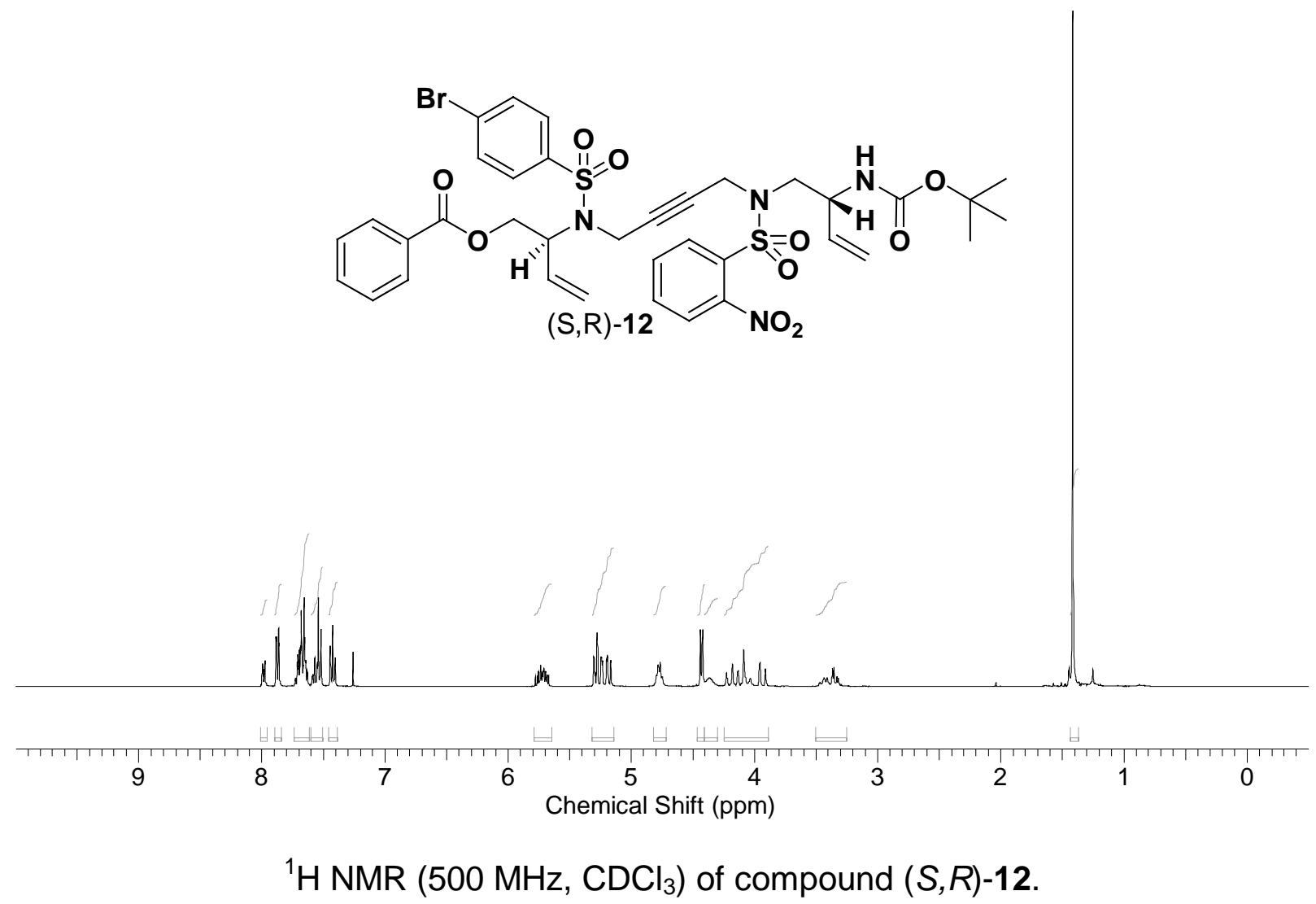




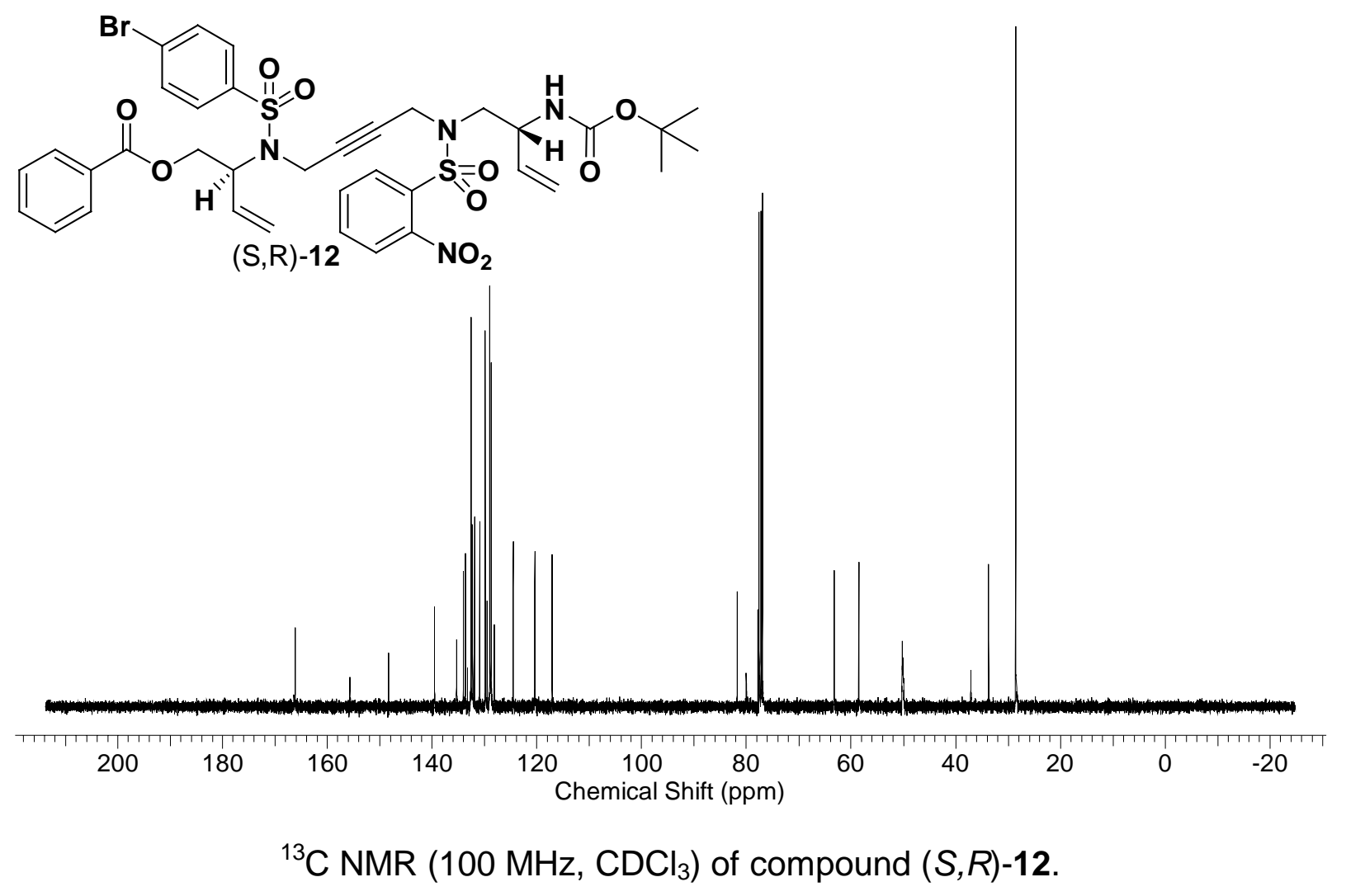

Spectral Data for N-Boc-ene-yne-ene (S,S)-12.

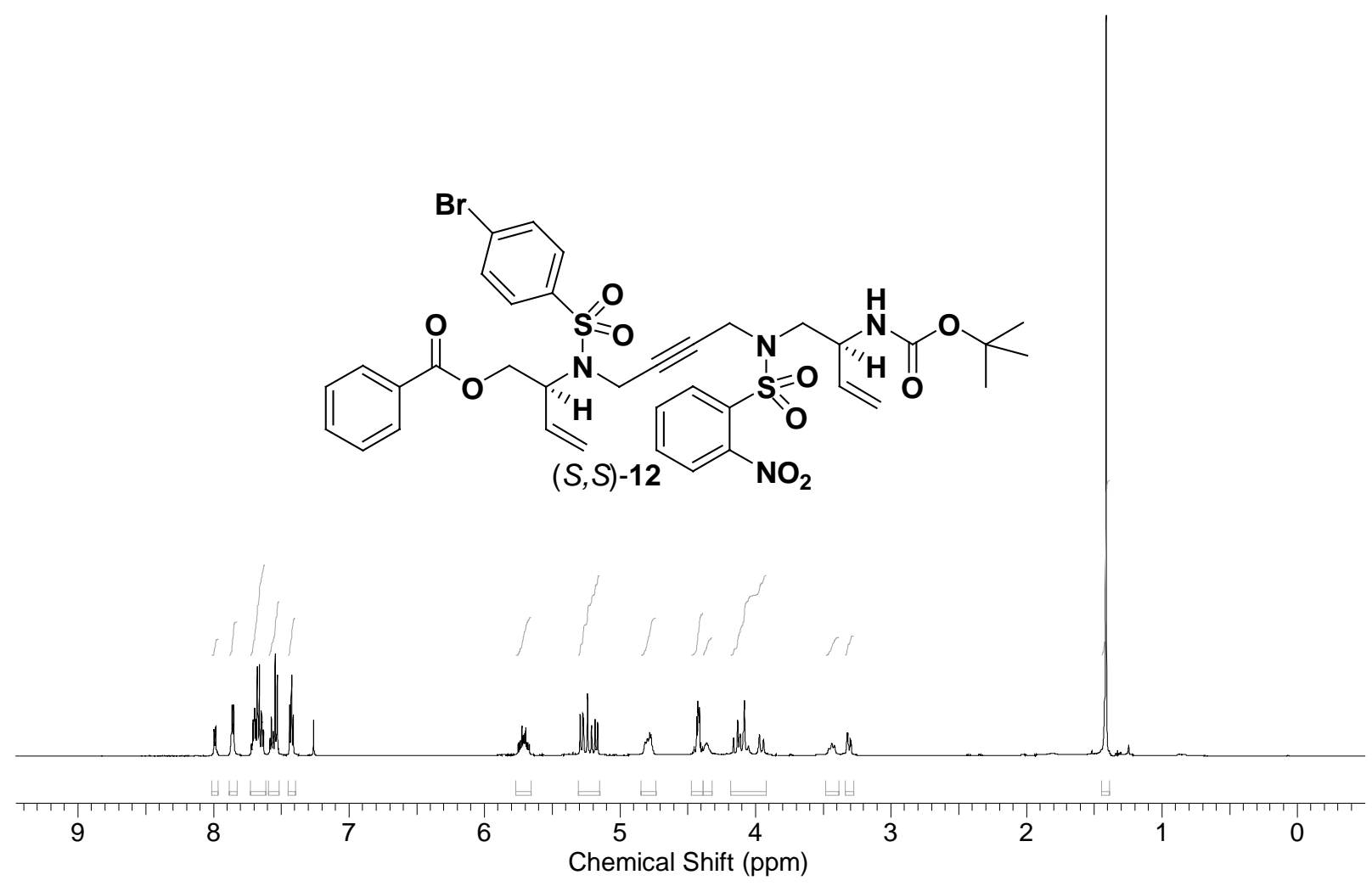

${ }^{1} \mathrm{H}$ NMR (500 MHz, $\mathrm{CDCl}_{3}$ ) of compound (S,S)-12. 


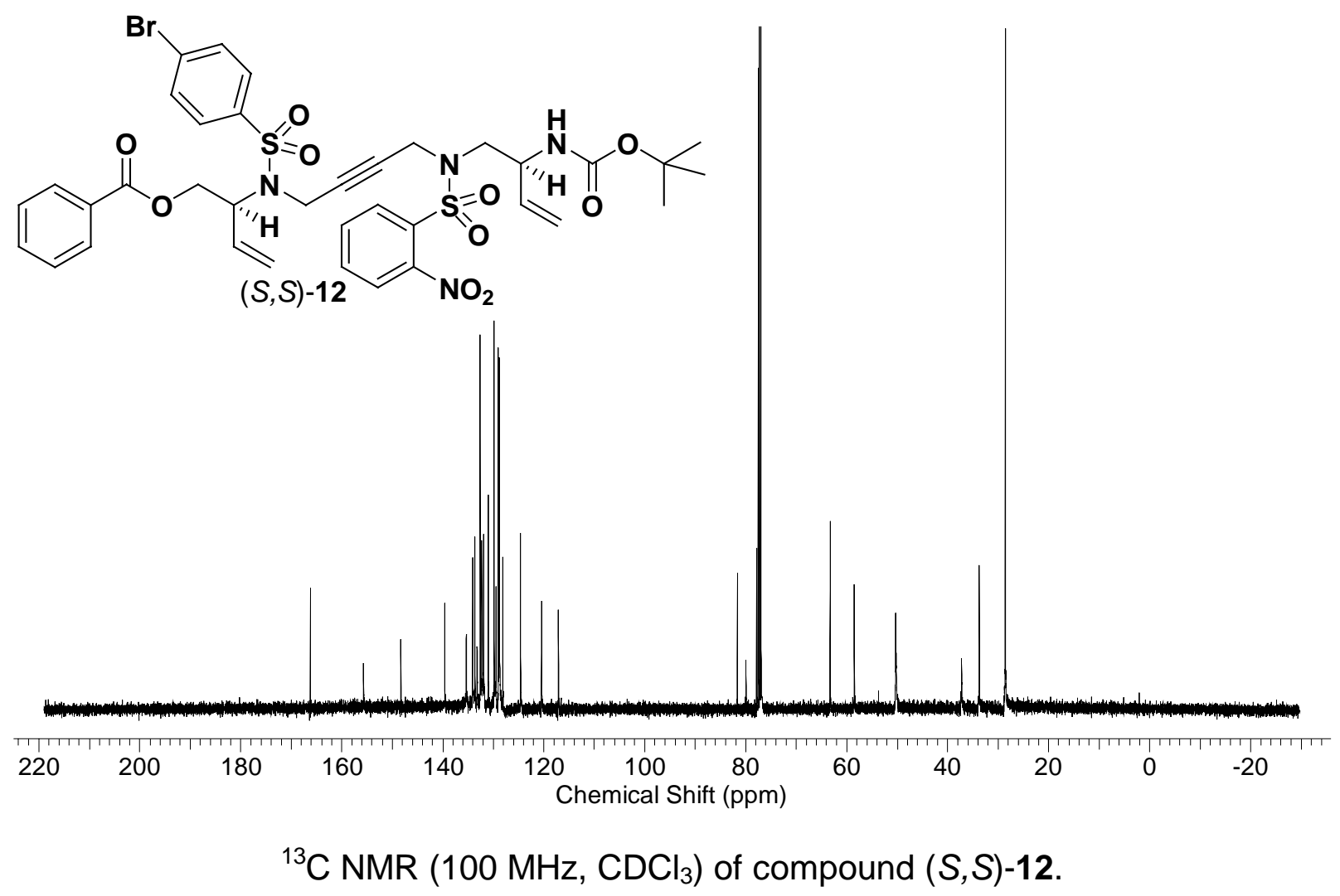

Spectral Data for (rac)-N-Boc-yne-yne-ene 13.

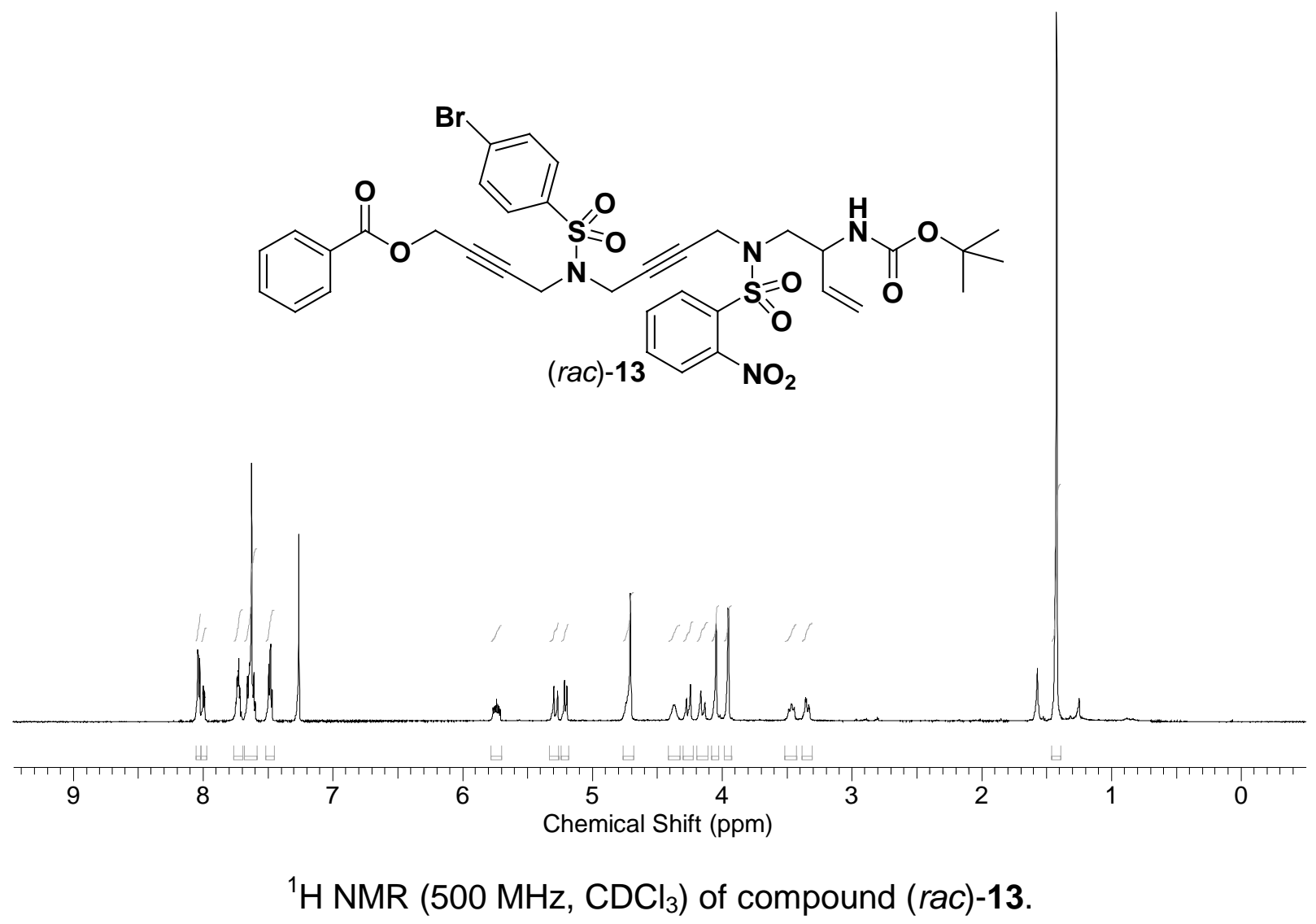




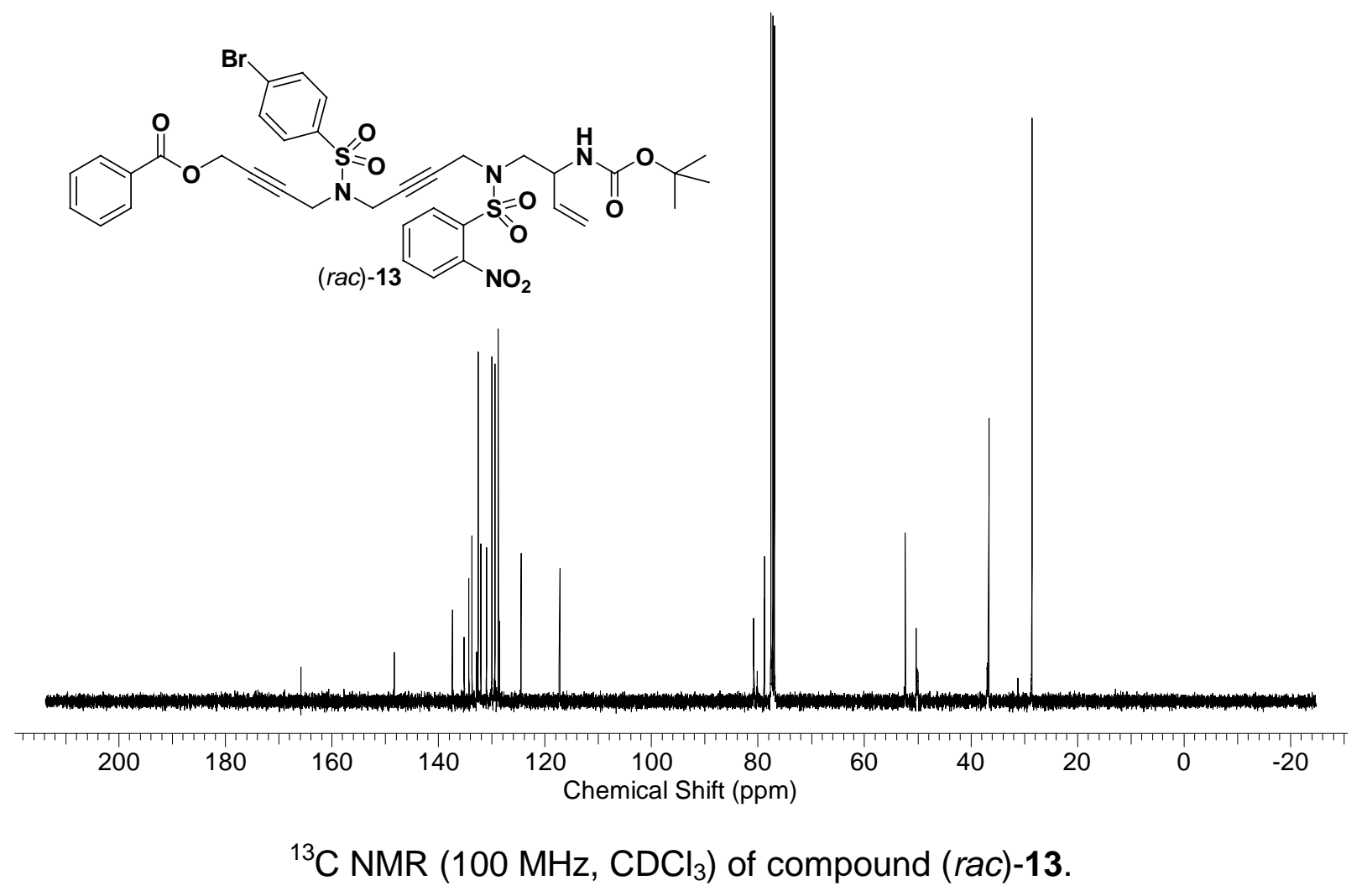

Spectral Data for Bicycle $(S, R)-14$.

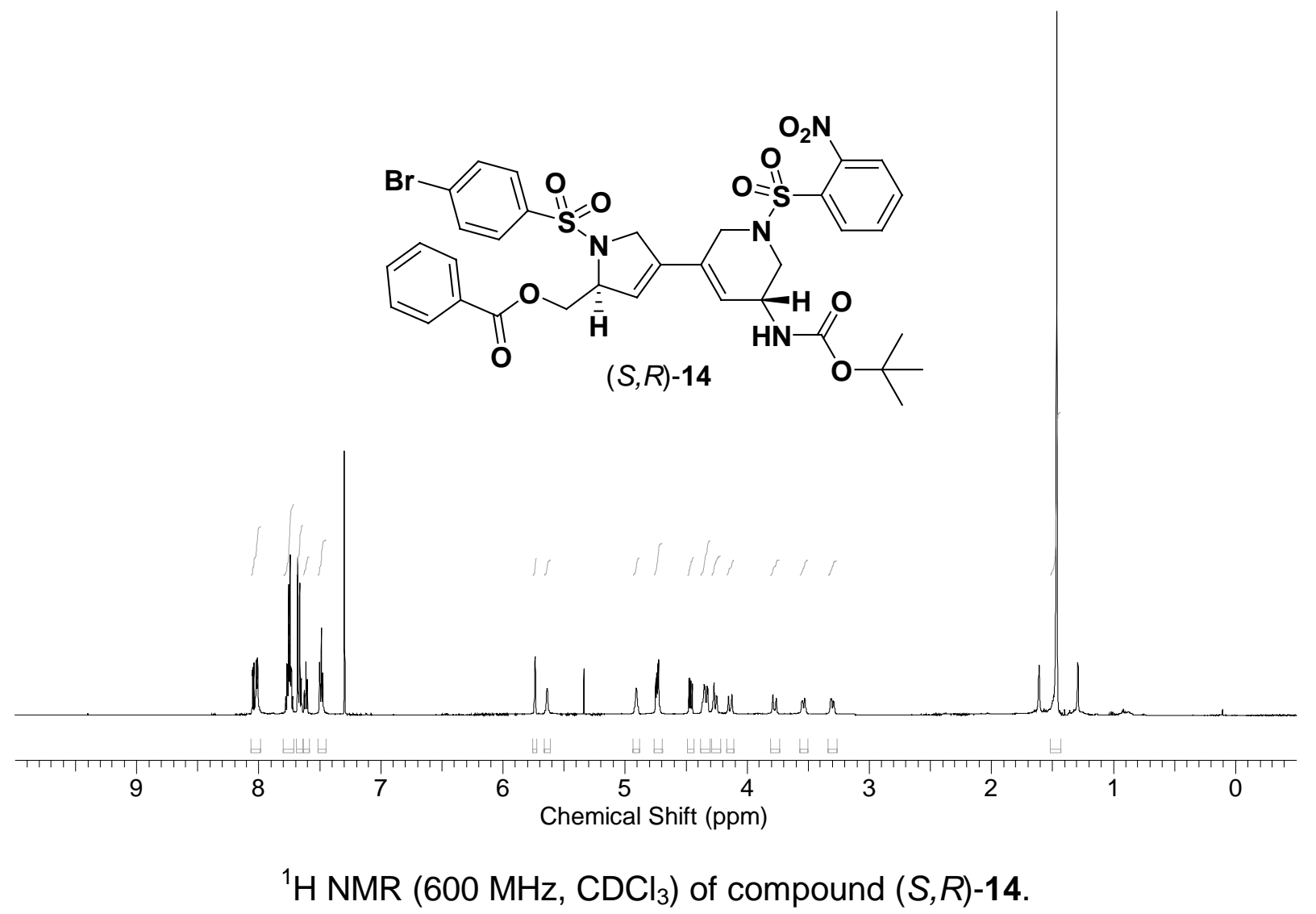




\section{Spectral Data for Bicycle (S,S)-14.}
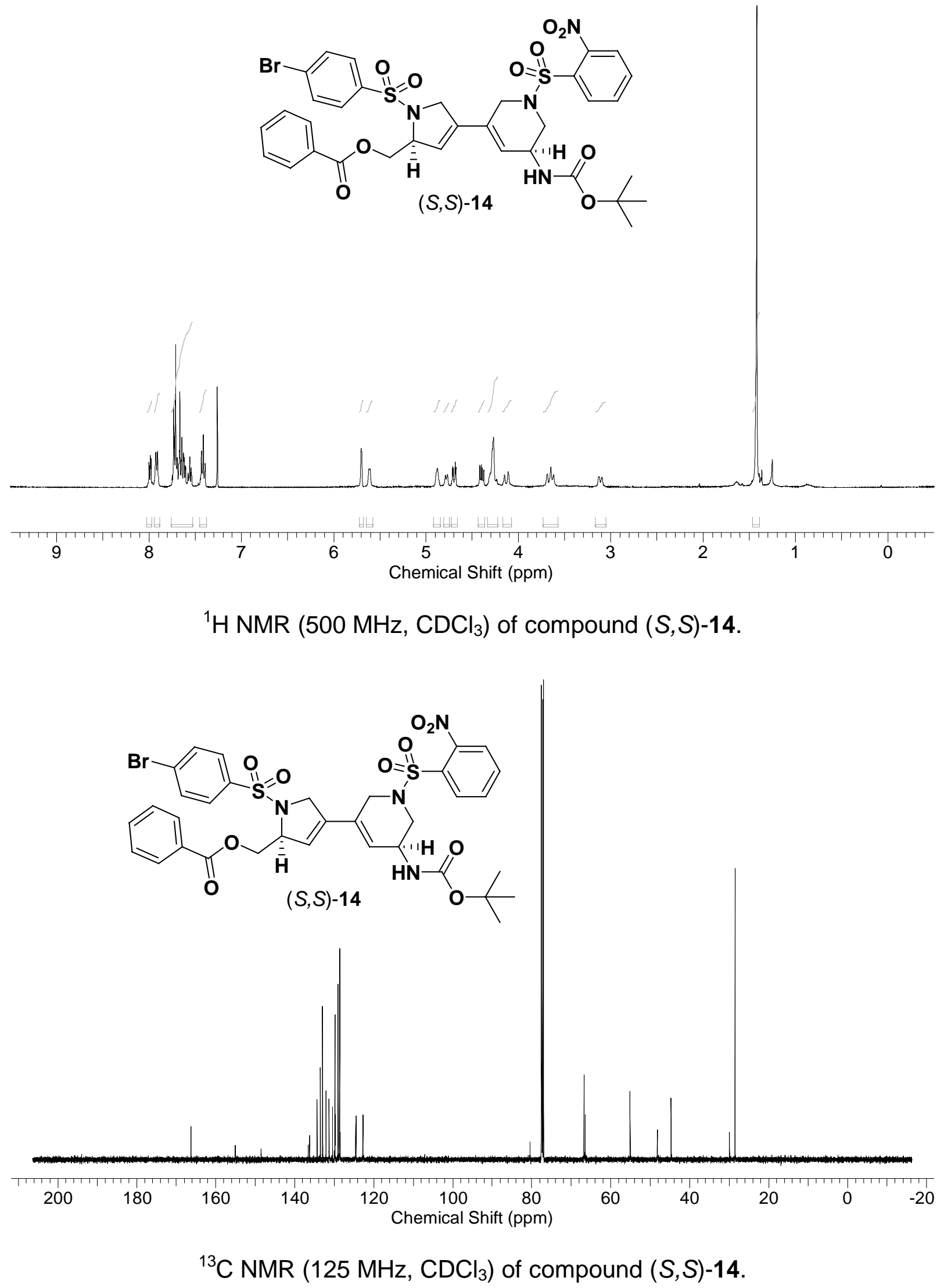

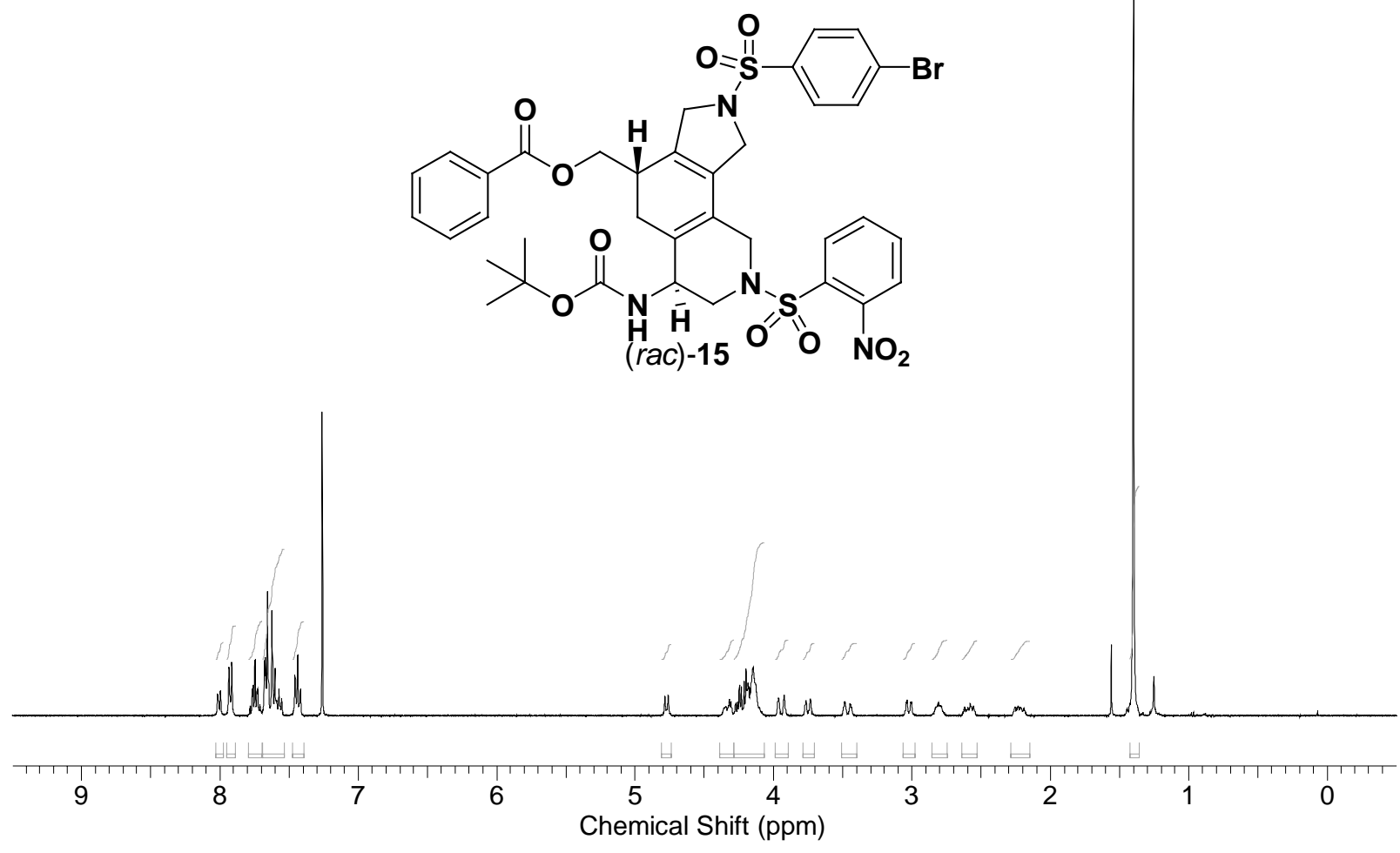

${ }^{1} \mathrm{H}$ NMR (500 MHz, $\mathrm{CDCl}_{3}$ ) of compound (rac)-15.<smiles>CC(C)(C)OC(=O)NC1CN(S(=O)(=O)c2ccccc2[N+](=O)[O-])CC2=C1C[C@H](COC(=O)c1ccccc1)C1=C2CN(S(=O)(=O)c2ccc(Br)cc2)C1</smiles>

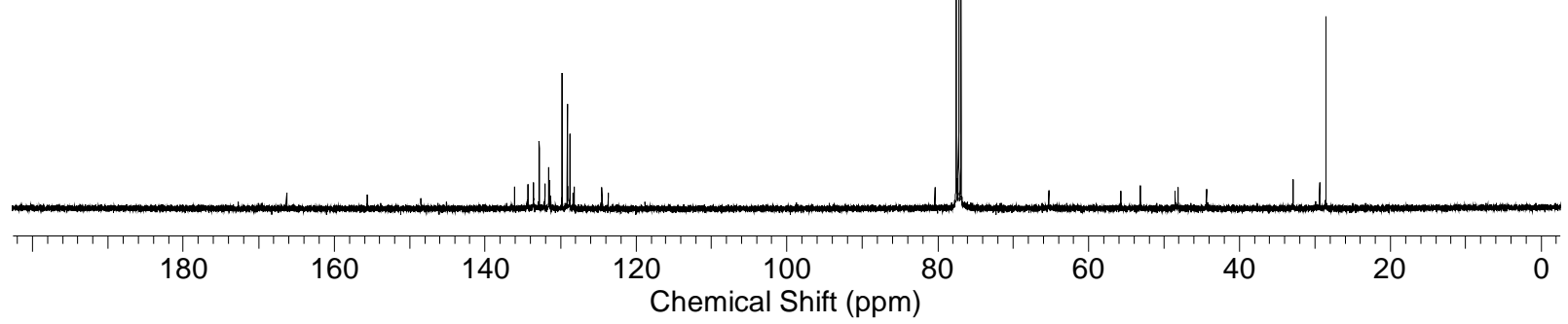

${ }^{13} \mathrm{C}$ NMR (125 MHz, $\mathrm{CDCl}_{3}$ ) of compound (rac)-15. 
Spectral Data for mixture of 15 with epi-15.
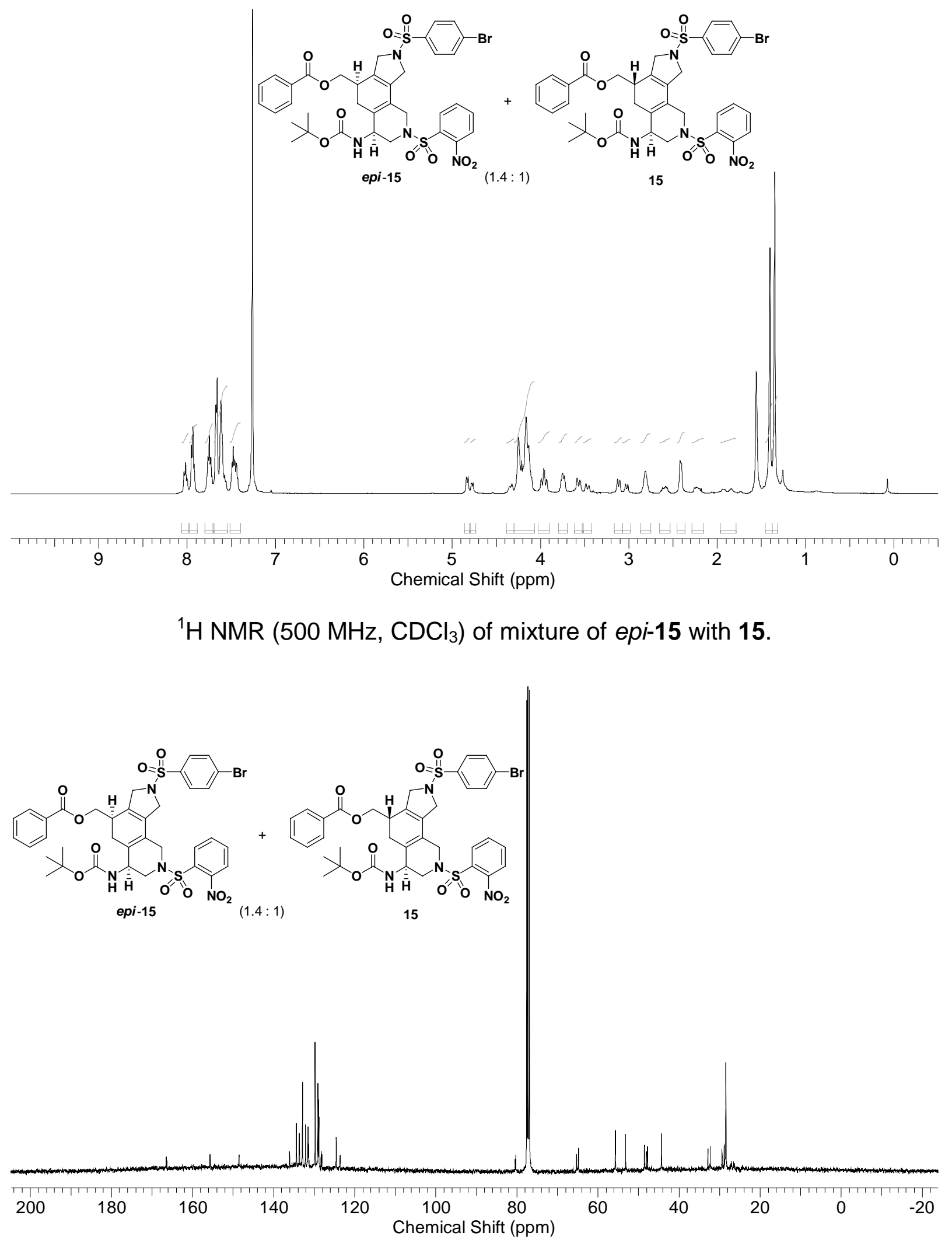

${ }^{13} \mathrm{C}$ NMR (125 MHz, $\mathrm{CDCl}_{3}$ ) of compound of epi-15 with 15. 
Spectral Data for Alcohol A7.

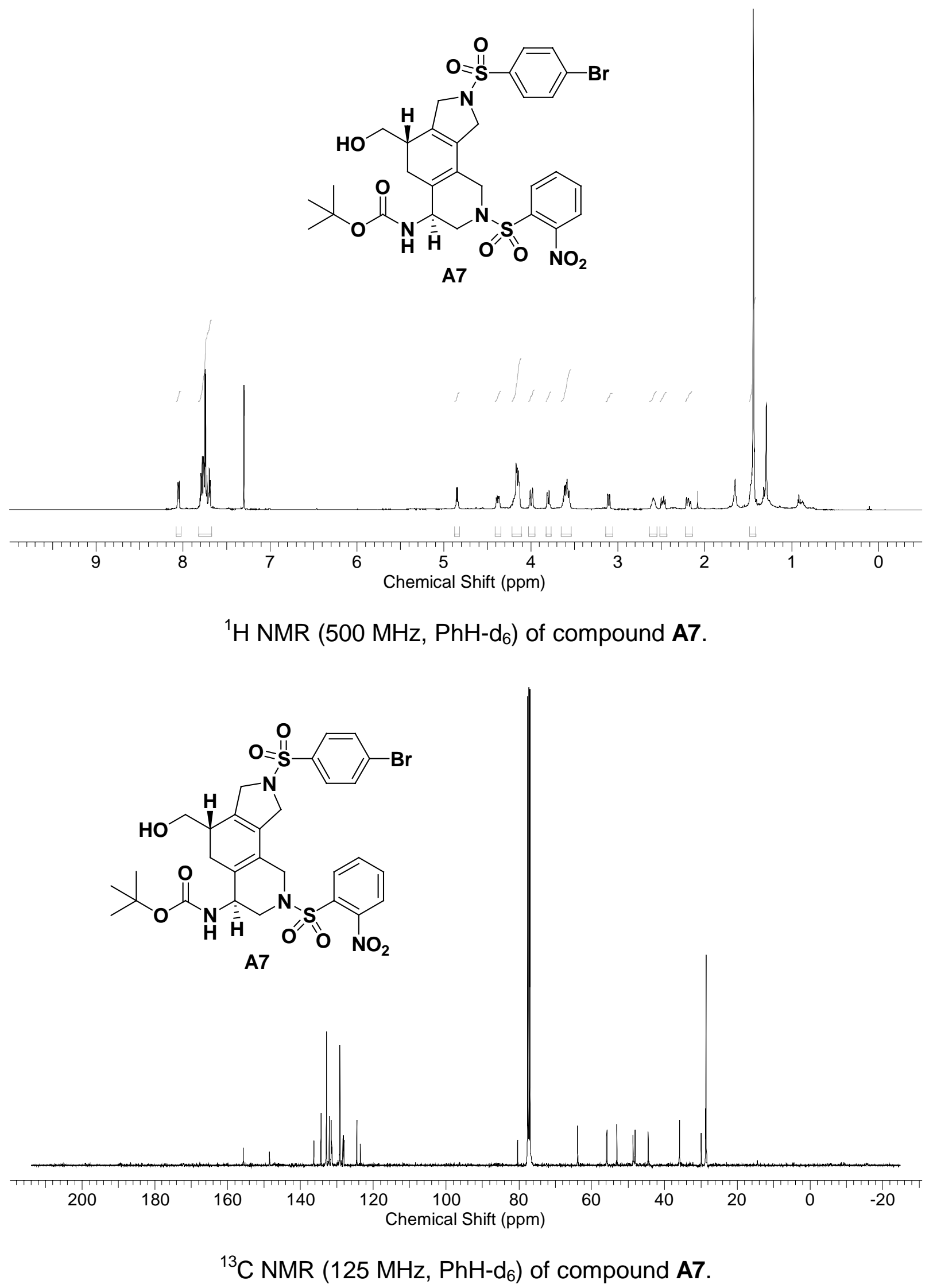


Spectral Data for Fused Cycloadduct 18.

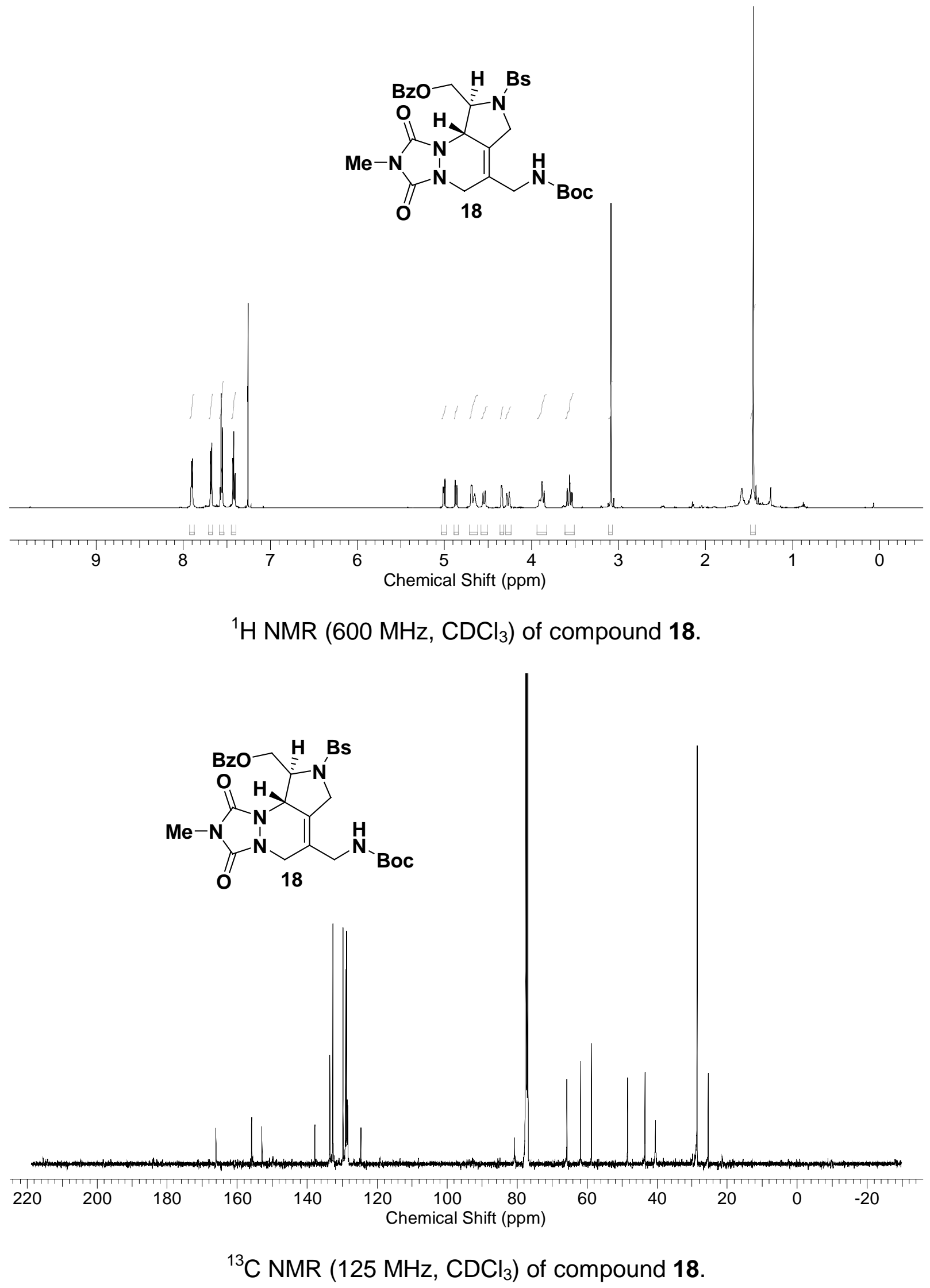


Spectral Data for Fused Cycloadduct 19 (high Rf diastereomer).

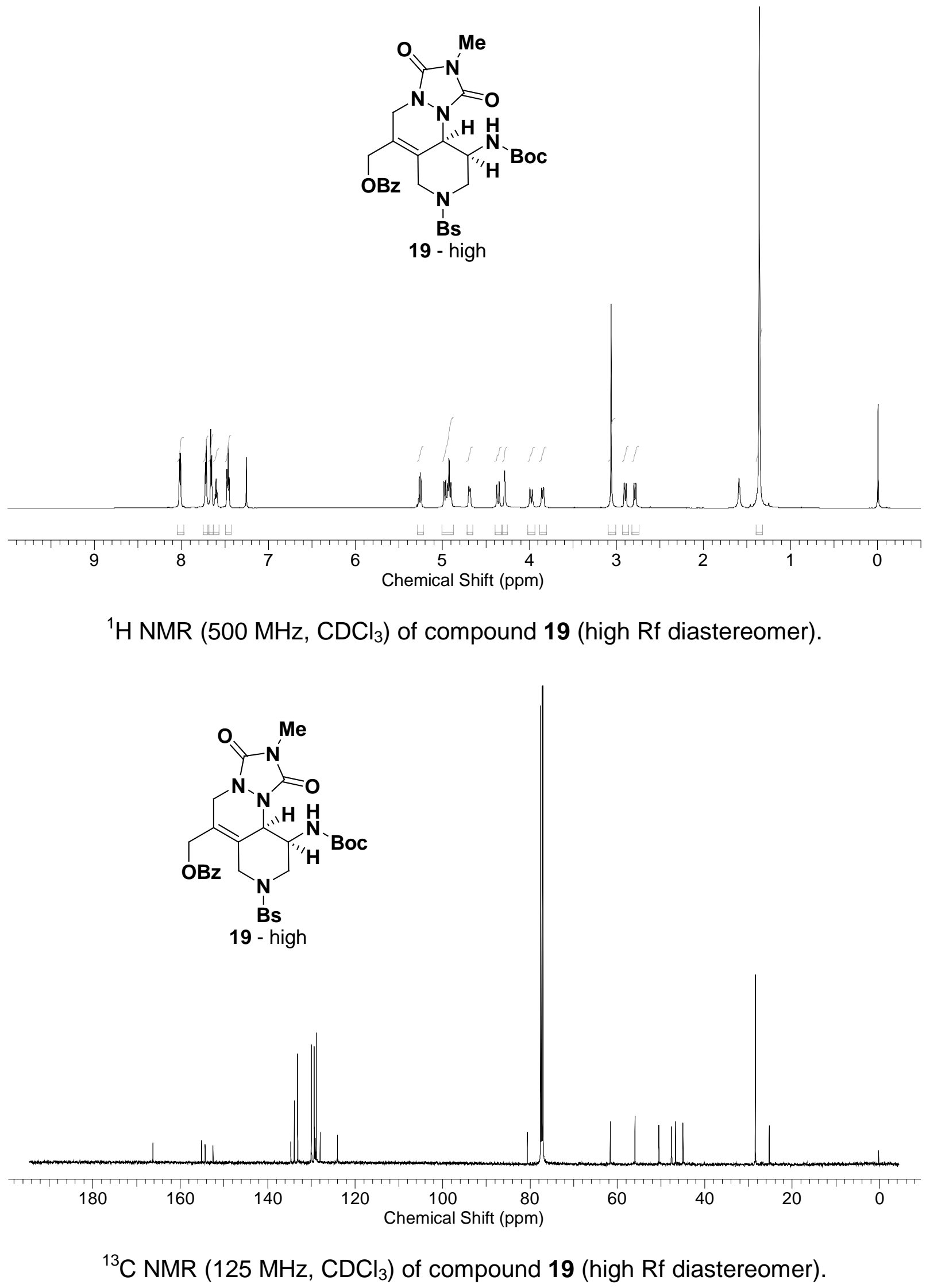


Spectral Data for Fused Cycloadduct 19 (low Rf diastereomer).
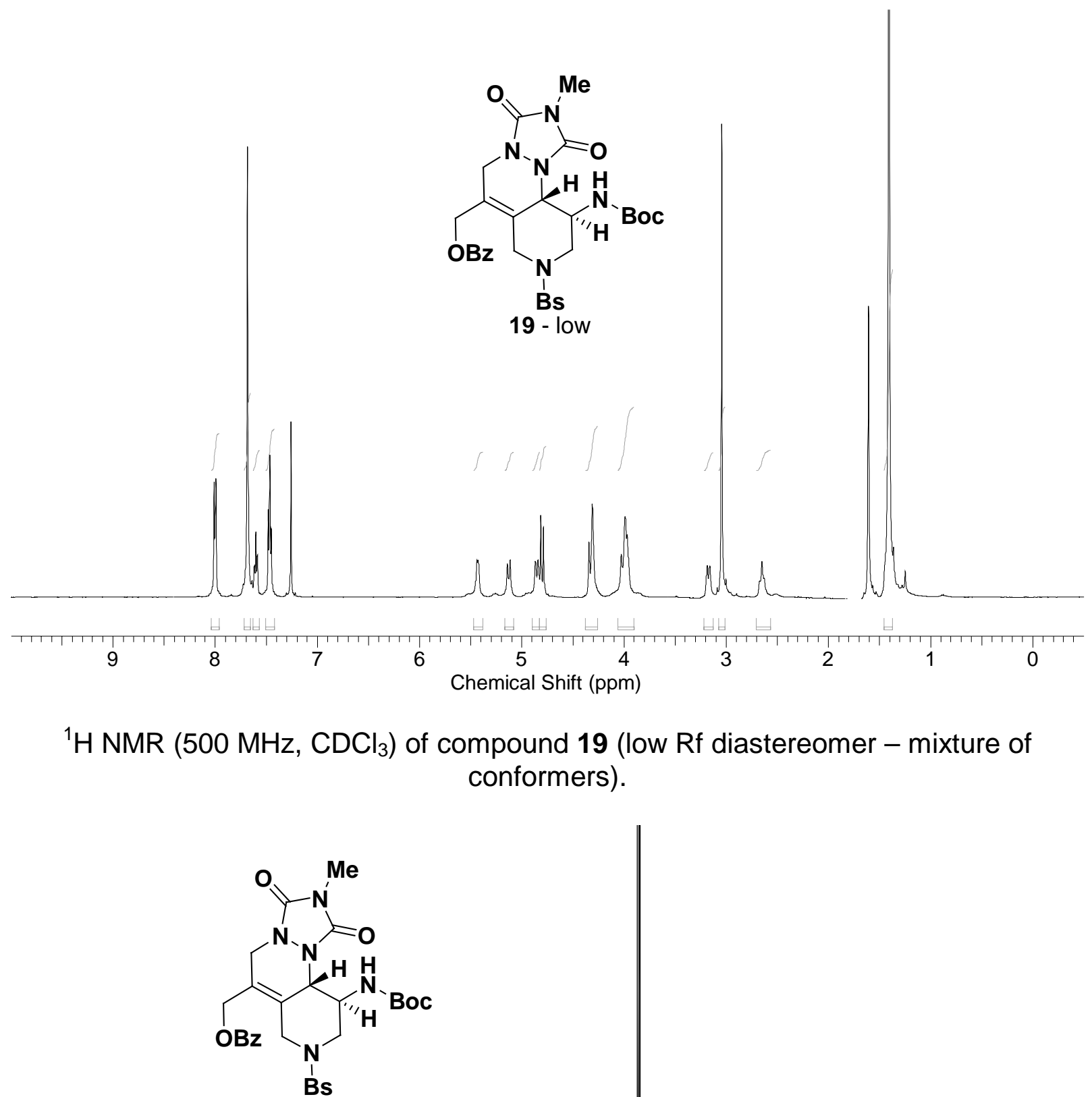

19 - low

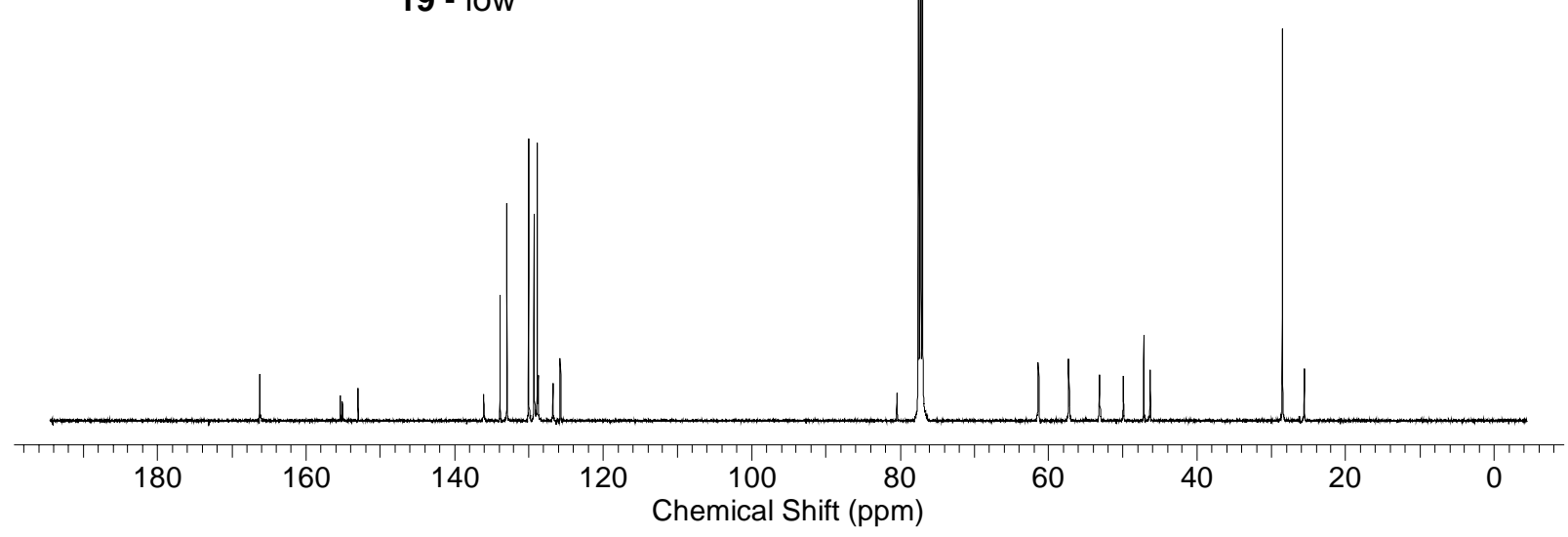

${ }^{13} \mathrm{C}$ NMR (125 MHz, $\mathrm{CDCl}_{3}$ ) of compound 19 (low Rf diastereomer). 
Spectral Data for Fused Tetracycle 20.

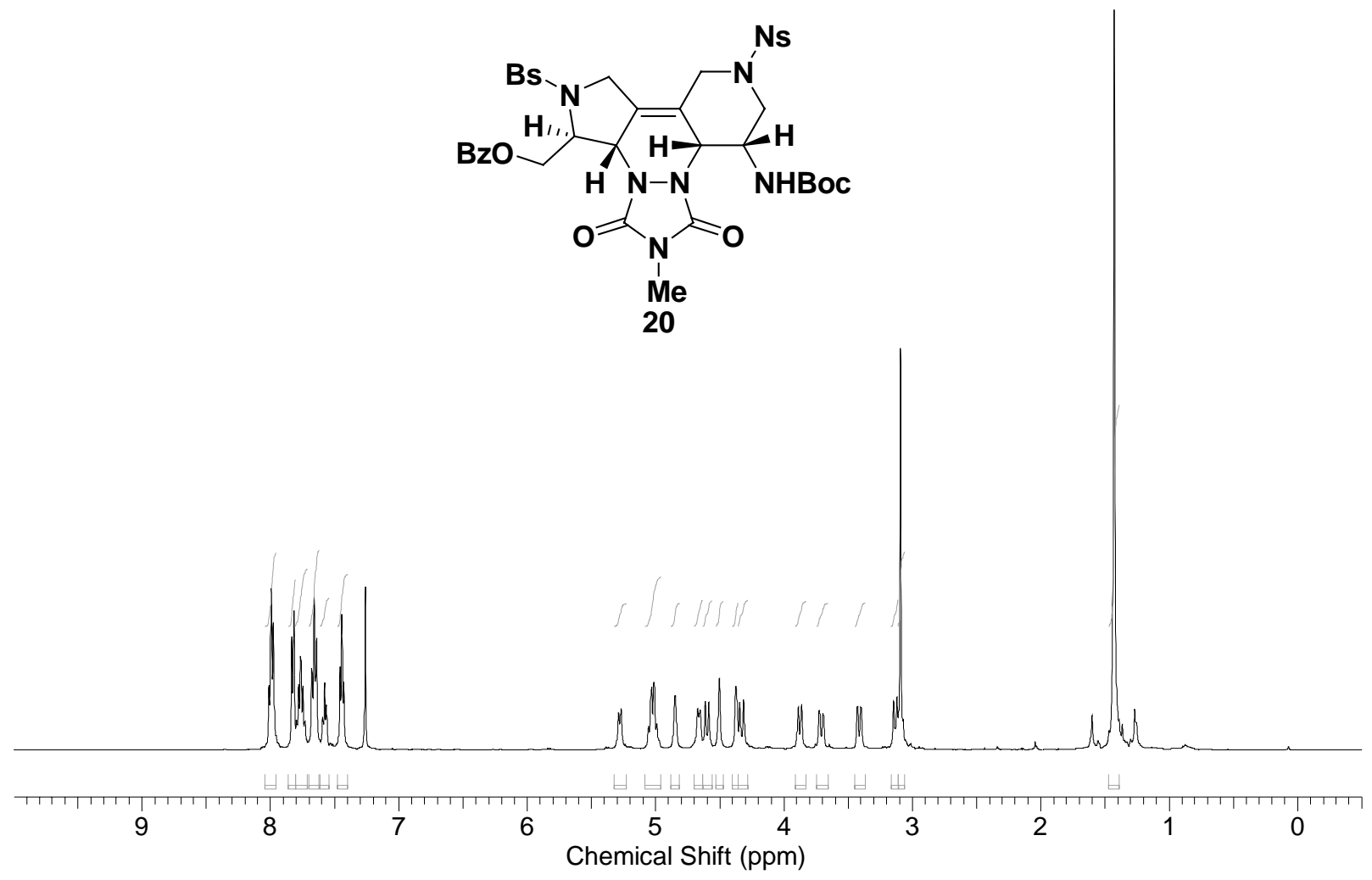

${ }^{1} \mathrm{H}$ NMR (500 MHz, $\mathrm{CDCl}_{3}$ ) of compound 20.

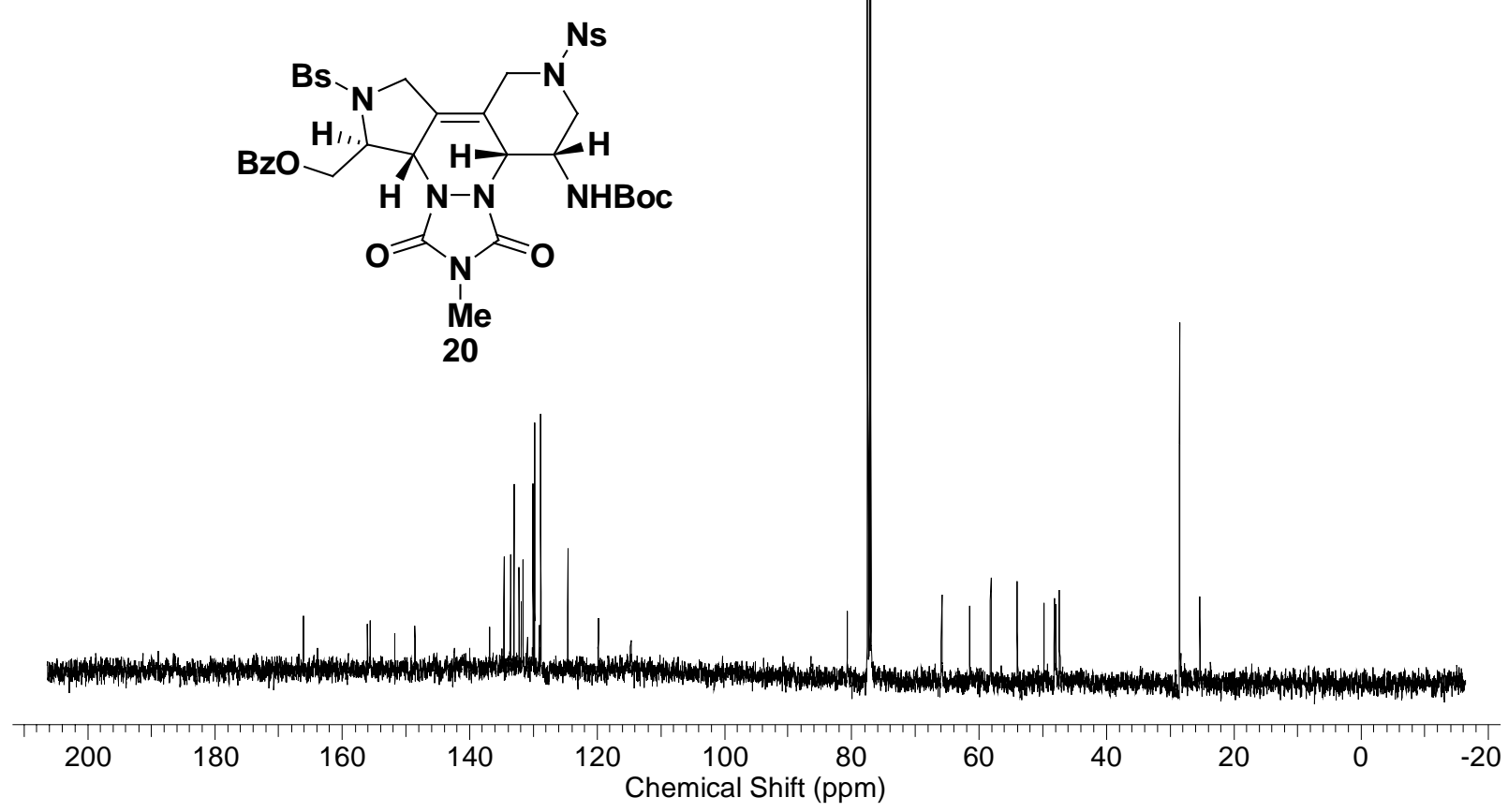

${ }^{13} \mathrm{C}$ NMR (125 MHz, $\mathrm{CDCl}_{3}$ ) of compound 22. 
Spectral Data for Fused Tetracycle 21.

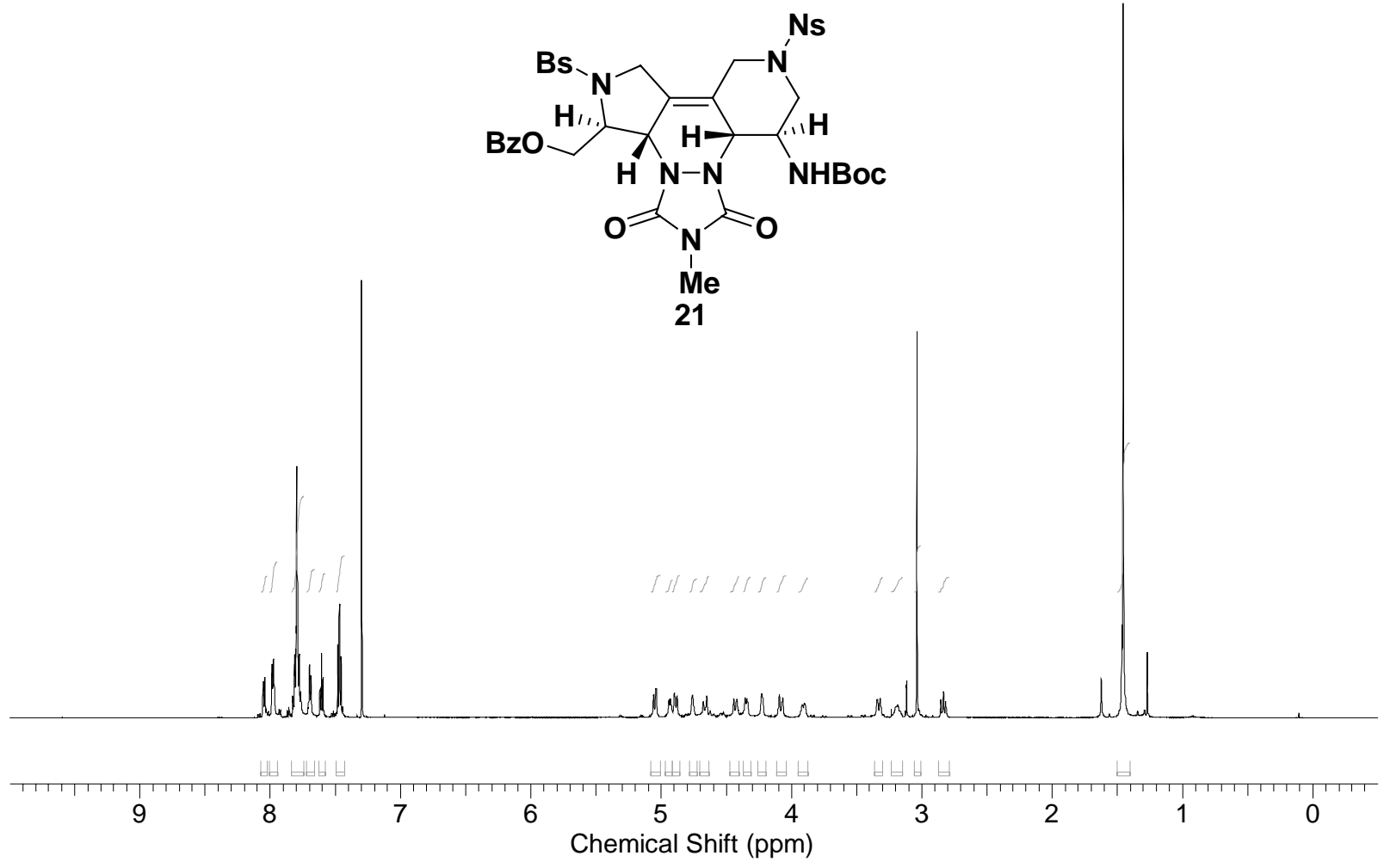

${ }^{1} \mathrm{H}$ NMR (500 MHz, $\mathrm{CDCl}_{3}$ ) of compound 21.
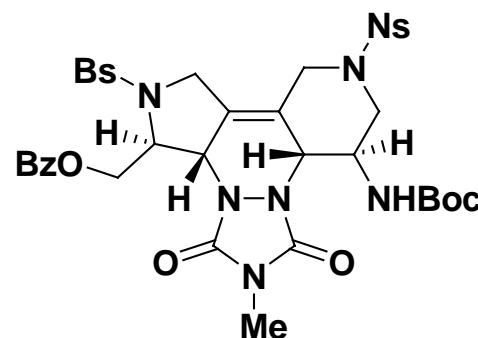

21

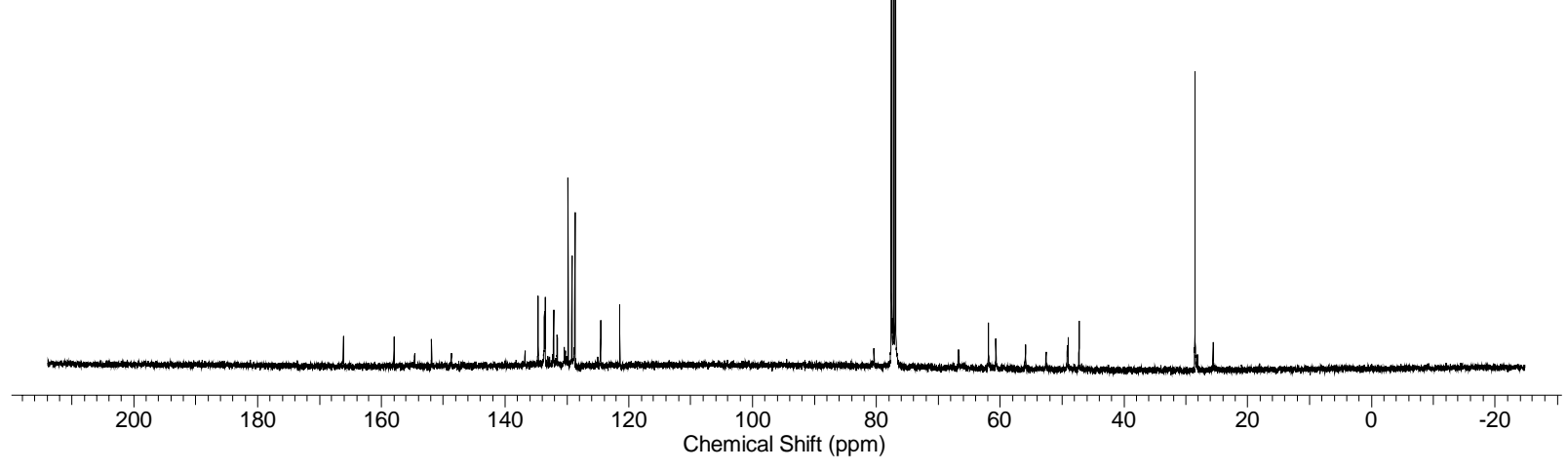

${ }^{13} \mathrm{C}$ NMR $\left(100 \mathrm{MHz}, \mathrm{CDCl}_{3}\right.$ ) of compound 21. 
Spectral Data for Bridged Pentacycle 22.

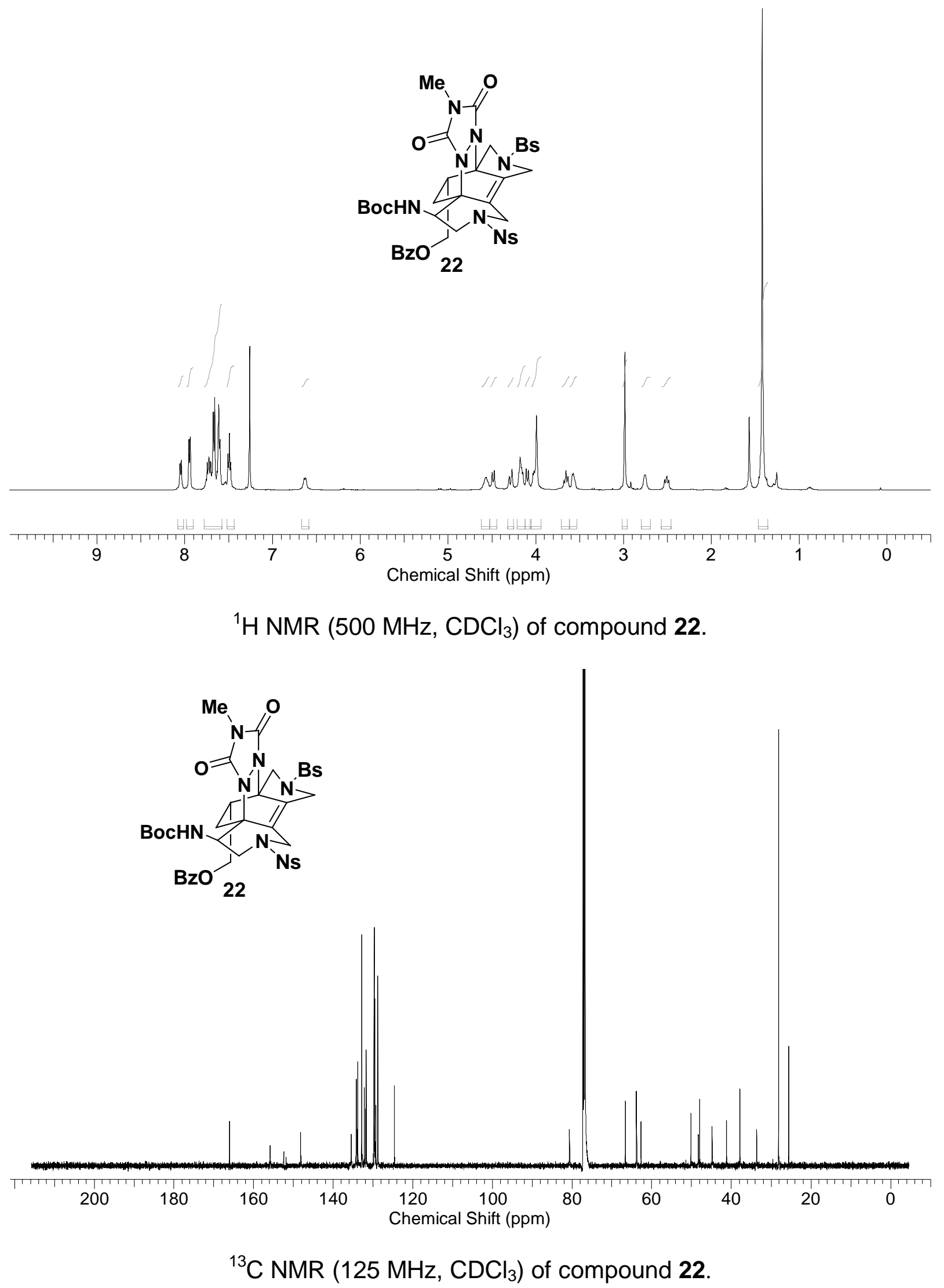

